\title{
A FINITE VOLUME METHOD FOR THE LAPLACE EQUATION ON ALMOST ARBITRARY TWO-DIMENSIONAL GRIDS
}

\author{
Komla Domelevo ${ }^{1}$ and Pascal Omnes $^{2}$
}

\begin{abstract}
We present a finite volume method based on the integration of the Laplace equation on both the cells of a primal almost arbitrary two-dimensional mesh and those of a dual mesh obtained by joining the centers of the cells of the primal mesh. The key ingredient is the definition of discrete gradient and divergence operators verifying a discrete Green formula. This method generalizes an existing finite volume method that requires "Voronoi-type" meshes. We show the equivalence of this finite volume method with a non-conforming finite element method with basis functions being $P^{1}$ on the cells, generally called "diamond-cells", of a third mesh. Under geometrical conditions on these diamondcells, we prove a first-order convergence both in the $\mathrm{H}_{0}^{1}$ norm and in the $\mathrm{L}^{2}$ norm. Superconvergence results are obtained on certain types of homothetically refined grids. Finally, numerical experiments confirm these results and also show second-order convergence in the $\mathrm{L}^{2}$ norm on general grids. They also indicate that this method performs particularly well for the approximation of the gradient of the solution, and may be used on degenerating triangular grids. An example of application on nonconforming locally refined grids is given.
\end{abstract}

Mathematics Subject Classification. 35J05, 35J25, 65N12, 65N15, 65N30.

Received: April 26, 2004. Revised: July 7, 2005.

\section{INTRODUCTION}

In this paper, we consider a finite volume method for the approximation of the Laplace equation:

$$
-\Delta \phi=f
$$

on a bounded domain $\Omega$, supplemented with adequate boundary conditions. Given a (primal) mesh covering $\Omega$, finite volume methods for this type of equation may be classified into two main distinct categories: "vertexcentered" methods and "cell-centered" methods. Vertex-centered methods compute approximate values of $\phi$ at the vertices of the primal mesh by integrating Equation (1) on dual cells associated to the vertices of the primal mesh. On the opposite, cell-centered methods compute approximate values of $\phi$ at the centers of the cells of the primal mesh by integrating Equation (1) on the primal cells. For a review of these methods, we

Keywords and phrases. Finite volume method, non-conforming finite element method, Laplace equation, discrete Green formula, diamond-cell, error estimates, convergence, superconvergence, arbitrary meshes, degenerating meshes, non-conforming meshes.

1 Mathématiques pour l'Industrie et la Physique, Université Paul Sabatier, 118 route de Narbonne, 31062 Toulouse Cedex 4 , France. komla@mip.ups-tlse.fr

2 Commissariat à l'Énergie Atomique, DEN-DM2S-SFME, 91191 Gif-sur-Yvette Cedex, France. pascal.omnes@cea.fr 
refer to [9]. In both cases, after using Gauss' formula, the values of $\nabla \phi \cdot \mathbf{n}$ on the sides of the (primal or dual) cells of the mesh have to be computed. If the line joining the centers of two adjacent cells is perpendicular to the corresponding interface, then the value of $\nabla \phi \cdot \mathbf{n}$ on this interface may simply be computed by a finite difference. This is the case for the so-called "admissible meshes" described in [9] which include Voronoi-type meshes (see also [20,25]). But for other general meshes (in particular for non-conforming meshes which can never be "admissible"), a possibility is to reconstruct the whole gradient, and not only its normal component (one may find alternative approaches to the reconstruction of the whole gradient in [11,19]). Several methods exist for this, which are partially reviewed in $[6,7]$. In these papers, the authors study the so-called "diamondcell" method to reconstruct the gradient. Each of these quadrilateral cells is associated with a side of the primal mesh and is obtained by joining the two vertices of this side with the centers of the two elements of the primal mesh which share this side. The mean-value of the gradient is defined with the help of the values of the function at the centers and at the vertices of the primal cells. The discrete solution at the vertices of the primal mesh is computed by a least-square linear interpolation of its values at the centers of the neighboring cells. The advantage of this scheme is that it can be defined on almost arbitrary two-dimensional grids and extends naturally to three dimensions. The main drawbacks are first, that the resulting numerical scheme is not symmetric, and thus more expensive iterative methods have to be employed to compute the solution of the associated linear system of equations; and second, that convergence of the discrete solution to the continuous one is obtained if the discrete system is coercive, which is proved only if the meshes verify some geometrical constraints ("almost-parallelogram" quadrangular meshes in the case of [6], and locally refined rectangular meshes with a lower and an upper bound on the aspect-ratio of the rectangles in the case of [7]).

In the present paper, we start by adopting the diamond-mesh methodology to reconstruct the gradient. But instead of interpolating the values of $\phi$ at the vertices of the primal mesh, we consider these values as supplementary unknowns of the numerical scheme. Therefore, we also have to write an equivalent number of supplementary equations. These are obtained by integrating the Laplace equation, not only on the cells of the primal mesh, but also on the cells of the dual mesh. Moreover, by doing so, we are in a position to define a discrete divergence operator (defined on the primal and dual meshes) which is the adjoint of the gradient operator (defined on the diamond mesh), which means that these two operators verify a discrete Green formula. Therefore, the scheme is by construction symmetric and coercive in the sense of [6] and belongs to the family of "support-operator methods" developed by Hyman and Shashkov in $[16,17]$. It also displays a variational form, which enables us to prove its equivalence with a non-standard non-conforming finite element method whose functions are piecewise $P^{1}$ on the diamond-cells and continuous only at the midpoints of the interfaces of these cells. It is also worth mentioning that if the sides of the primal and dual cells are orthogonal, then the scheme fully decouples into two independent schemes: the first only involves unknowns at the centers of the primal cells and the second only those at the vertices of the primal mesh and, in that case, both schemes are identical to the above-mentioned cell-centered and cell-vertex schemes, respectively. Thus, the presented scheme may be considered as a generalization of the schemes that use Voronoi-type meshes. We also remark that Hermeline [15] used a finite volume method, that turns out to be equivalent to that presented here, to approach more general linear and non-linear diffusion operators on distorted meshes. The originality of our approach is that the definitions of the discrete gradient and divergence operators lead to a scheme which is, by construction, positive definite, while this property had to be proved in [15]. Moreover, the equivalence with a non-conforming finite element method and its use on non-conforming meshes are also original properties. Finally, error estimates for this scheme are provided for the first time, assuming the diamond-cells of the mesh respect some geometrical hypotheses. These hypotheses explain why this scheme is particularly adapted to very distorted or degenerating grids. In the case of the traditional Lagrange finite elements, it is well-known that a quality criterion of the grid is given by the so-called "maximum angle condition" (see $[2,18]$ ): The optimal rate of convergence of the numerical solution toward the solution of the continuous problem, on a family of grids whose step $h$ tends to 0 , is obtained if, and only if, the maximum angle of the triangles is bounded away from $\pi$, uniformly in $h$. This property was then extended by Acosta and Durán to the mixed finite elements of Raviart-Thomas type and to the non-conforming finite elements of Crouzeix-Raviart type in [1]. As far as the scheme presented here is 
concerned, we obtain a sufficient condition of convergence related to the angles of the diamond-cells: each one of these quadrangles can be split along one of its diagonals into two triangles. According to the diagonal we chose, we obtain two couples of triangles (see Fig. 6). The convergence of the scheme with an order $h$ in a discrete $\mathrm{H}_{0}^{1}$ norm, as well as in the $\mathrm{L}^{2}$ norm, is obtained if the maximum angle of the triangles of one of these two couples is bounded away from $\pi$, uniformly in $h$. In addition, if the family of meshes is obtained through homothetic refinement, then we can prove that the scheme is of order $1.5-\alpha$ in the $\mathrm{H}_{0}^{1}$ norm and of order $2-\alpha$ in the $\mathrm{L}^{2}$ norm (for all $\alpha>0$ ) if the data $f$ is in $\mathrm{H}^{1}(\Omega)$. The presented numerical experiments confirm the theoretical results in norm $\mathrm{H}_{0}^{1}$ and show in addition that a second-order convergence in the $\mathrm{L}^{2}$ norm takes place even if the diamond-cells are not parallelograms.

The rest of the paper is organized as follows: in Section 1, we introduce our notations; in Section 2, we define the discrete gradient operator on the diamond-cells and the corresponding adjoint discrete divergence operator on the cells of the primal and dual meshes. In Section 3, we write down the finite volume scheme and describe some of its properties. In Section 4, we prove its equivalence with a non-conforming finite element scheme. In Section 5, we study the numerical error in a discrete $\mathrm{H}_{0}^{1}$ norm by distinguishing an approach close to that presented in [9], which we name "finite volume approach" and a more standard finite element approach. Further, in Section 6, we derive an estimate in the $\mathrm{L}^{2}$ norm, while in Section 7, we give superconvergence results on homothetically refined triangular grids. We conclude this work by presenting in Section 8 convergence curves in the discrete $\mathrm{H}_{0}^{1}$ and $\mathrm{L}^{2}$ norms on various types of grids: triangular, homothetically refined triangular, degenerating triangular, and finally locally refined non-conforming meshes.

\section{Definitions And nOtations}

First, we shall denote by $\hat{\phi}$ the exact solution of Equation (1).

The following notations are summarized in Table 1:

Let $\Omega$ be a bounded connected polygon of $\mathbb{R}^{2}$, whose boundary is denoted by $\Gamma$, covered by a mesh (named primal mesh) composed of elements $T_{i}$, with $i \in[1, I]$. These elements are supposed to be convex polygons that form a partition of $\Omega$. With each element $T_{i}$ of the mesh, we associate a node $G_{i}$ located inside $T_{i}$. This point may be the barycenter of $T_{i}$, but this is not necessary. The area of $T_{i}$ is denoted by $\left|T_{i}\right|$. The characteristic function of the cell $T_{i}$ will be denoted by $\theta_{i}^{T}$.

We shall denote by $J$ the total number of sides of this mesh and by $J^{\Gamma}$ the number of these sides which are located on the boundary $\Gamma$. The sides of the mesh are denoted by $A_{j}$ and their lengths by $\left|A_{j}\right|$, with $j \in[1, J]$. We further suppose that the set $[1, J]$ is ordered so that when $A_{j}$ is not located on $\Gamma$, then $j \in\left[1, J-J^{\Gamma}\right]$ and when $A_{j}$ is on $\Gamma$, then $j \in\left[J-J^{\Gamma}+1, J\right]$. With each of these boundary sides, we associate its midpoint, also denoted by $G_{i}$ with $i \in\left[I+1, I+J^{\Gamma}\right]$. For those $i \in\left[I+1, I+J^{\Gamma}\right]$, we then denote by $j(i)$ the subscript of the associated side $A_{j}$.

Further, we denote by $S_{k}$, with $k \in[1, K]$, the nodes of the polygons of the primal mesh. To each of these points, we associate a polygon denoted by $P_{k}$, obtained by joining the points $G_{i}$ associated to the elements of the primal mesh (and possibly to the boundary sides) of which $S_{k}$ is a node. The area of $P_{k}$ is denoted by $\left|P_{k}\right|$. We shall only consider in the following the cases where the dual cells $P_{k}$ constitute a second partition of $\Omega$, which we name dual mesh ${ }^{1}$. Figure 1 displays an example of a non-conforming primal mesh and its associated dual mesh. We further suppose that the set $[1, K]$ is ordered so that when $S_{k}$ is not on $\Gamma$, then $k \in\left[1, K-J^{\Gamma}\right]$ and when $S_{k}$ is on $\Gamma$, then $k \in\left[K-J^{\Gamma}+1, K\right]$. For those $k \in\left[K-J^{\Gamma}+1, K\right]$, we define $\tilde{A}_{k}$ as the intersection of the boundary $\Gamma$ with the boundary of $P_{k}$ (see Fig. 3 ) and $\tilde{\mathbf{n}}_{k}$ as the outgoing normal unit vector on $\tilde{A}_{k}$. The characteristic function of the cell $P_{k}$ will be denoted by $\theta_{k}^{P}$.

With each side of the primal mesh, we associate a quadrilateral named "diamond-cell" and denoted by $D_{j}$. When $A_{j}$ is not on the boundary, this cell is obtained by joining the points $S_{k_{1}(j)}$ and $S_{k_{2}(j)}$, which are the two nodes of $A_{j}$, with the points $G_{i_{1}(j)}$ and $G_{i_{2}(j)}$ associated to the elements of the primal mesh which share this side. When $A_{j}$ is on the boundary $\Gamma$, the cell $D_{j}$ is obtained by joining the two nodes of $A_{j}$ with the point

\footnotetext{
${ }^{1}$ It may happen that the dual cells overlap, as shown in Figure 2.
} 
TABLE 1. List of notations.

\begin{tabular}{|c|c|c|}
\hline Symbol & Description & Figure \\
\hline$I$ & Number of cells in the primal mesh & - \\
\hline$J$ & Number of sides of the primal mesh & - \\
\hline$J^{\Gamma}$ & $\begin{array}{l}\text { Number of sides of the primal mesh located on the boundary } \Gamma \\
\text { (by convention } j \in\left[J-J^{\Gamma}+1, J\right] \text { if the side } j \text { is on } \Gamma \text { ) }\end{array}$ & - \\
\hline$K$ & $\begin{array}{l}\text { Number of nodes of the primal mesh }=\text { Number of dual cells } \\
\text { (by convention } k \in\left[K-J^{\Gamma}+1, K\right] \text { iff the node } k \text { is on } \Gamma \text { ) }\end{array}$ & - \\
\hline$T_{i}, i \in[1, I]$ & Cell of the primal mesh & Fig. 1 \\
\hline$\left|T_{i}\right|$ & Area of $T_{i}$ & \\
\hline$\theta_{i}^{T}, i \in[1, I]$ & Characteristic function of the primal cell $T_{i}$ & - \\
\hline$G_{i}, i \in[1, I]$ & Point associated to the primal cell $T_{i}$ & Fig. 1 \\
\hline$G_{i}, i \in\left[I+1, I+J^{\Gamma}\right]$ & Midpoint of a side located on $\Gamma$ & - \\
\hline$j(i), i \in\left[I+1, I+J^{\Gamma}\right]$ & Subscript of the boundary side associated with the boundary point $G_{i}$ & - \\
\hline$P_{k}, k \in[1, K]$ & Cell of the dual mesh & Fig. 1 \\
\hline$\left|P_{k}\right|$ & Area of $P_{k}$ & - \\
\hline$\theta_{k}^{P}, k \in[1, K]$ & Characteristic function of the dual cell $P_{k}$ & - \\
\hline$S_{k}, k \in[1, K]$ & Node of the primal mesh $=$ Point associated to the dual cell $P_{k}$ & Fig. 1 \\
\hline$\tilde{A}_{k}, k \in\left[K-J^{\Gamma}+1, K\right]$ & Intersection of $\Gamma$ and of the boundary of the dual cell $P_{k}$ & Fig. 3 \\
\hline$\tilde{\mathbf{n}}_{k}, k \in\left[K-J^{\Gamma}+1, K\right]$ & Outgoing unit normal vector on $\tilde{A}_{k}$ & Fig. 3 \\
\hline$D_{j}, j \in[1, J]$ & Cell of the diamond mesh & Fig. 4 \\
\hline$\left|D_{j}\right|, j \in[1, J]$ & Area of $D_{j}$ & - \\
\hline$i_{1}(j), i_{2}(j)$ & Subscript of the primal cells which share $A_{j}$ as a common edge & Fig. 4 \\
\hline$k_{1}(j), k_{2}(j)$ & Subscript of the mesh nodes which are the vertices of the side $A_{j}$ & Fig. 4 \\
\hline$G_{i_{\alpha}(j)},(\alpha, \beta) \in\{1 ; 2\}^{2}$ & Vertices of the diamond cell $D_{j}$ associated to the primal cells $T_{i_{\alpha}(j)}$ & Fig. 4 \\
\hline$S_{k_{\beta}(j)},(\alpha, \beta) \in\{1 ; 2\}^{2}$ & Vertices of the diamond cell $D_{j}$ associated to the dual cells $P_{k_{\beta}(j)}$ & Fig. 4 \\
\hline $\begin{array}{l}A_{j}=\left[S_{k_{1}(j)} S_{k_{2}(j)}\right] \\
\left|A_{j}\right|\end{array}$ & $\begin{array}{l}\text { Side of the primal mesh }=\text { Diagonal of the diamond cell } D_{j} \\
\text { Length of } A_{j}\end{array}$ & Fig. 5 \\
\hline$A_{j}^{\prime}=\left[G_{i_{1}(j)} G_{i_{2}(j)}\right]$ & Side of the dual mesh $=$ Diagonal of the diamond cell $D_{j}$ & Fig. 5 \\
\hline & Length of $A_{j}^{\prime}$ & - \\
\hline$M_{i_{\alpha} k_{\beta}},(\alpha, \beta) \in\{1 ; 2\}^{2}$ & Midpoint of the side $\left[G_{i_{\alpha}(j)} S_{k_{\beta}(j)}\right]$ of the diamond cell $D_{j}$ & Fig. 5 \\
\hline $\mathbf{n}_{j}, j \in[1, J]$ & Unit normal vector to $A_{j}$, oriented so that $\mathbf{G}_{\mathbf{i}_{\mathbf{1}}(\mathbf{j})} \mathbf{G}_{\mathbf{i}_{\mathbf{2}}(\mathbf{j})} \cdot \mathbf{n}_{j} \geq 0$ & Fig. 5 \\
\hline $\mathbf{n}_{j}^{\prime}, j \in[1, J]$ & Unit normal vector to $A_{j}^{\prime}$, oriented so that $\mathbf{S}_{\mathbf{k}_{\mathbf{1}}(\mathbf{j})} \mathbf{S}_{\mathbf{k}_{\mathbf{2}}(\mathbf{j})} \cdot \mathbf{n}_{j} \geq 0$ & Fig. 5 \\
\hline$D_{j, 1}, j \in[1, J]$ & The triangle $S_{k_{1}(j)} G_{i_{1}(j)} S_{k_{2}(j)}$ associated to the diamond cell $D_{j}$ & Fig. 6 \\
\hline$D_{j, 2}, j \in[1, J]$ & The triangle $S_{k_{2}(j)} G_{i_{2}(j)} S_{k_{1}(j)}$ associated to the diamond cell $D_{j}$ & Fig. 6 \\
\hline$D_{j, 1}^{\prime}, j \in[1, J]$ & The triangle $G_{i_{2}(j)} S_{k_{1}(j)} G_{i_{1}(j)}$ associated to the diamond cell $D_{j}$ & Fig. 6 \\
\hline$D_{j, 2}^{\prime}, j \in[1, J]$ & The triangle $G_{i_{1}(j)} S_{k_{2}(j)} G_{i_{2}(j)}$ associated to the diamond cell $D_{j}$ & Fig. 6 \\
\hline $\mathcal{T}_{j, 1}, j \in[1, J]$ & $\begin{array}{l}\text { Either } D_{j, 1} \text { or } D_{j, 1}^{\prime} \text { according to the local splitting of } D_{j} \\
\text { along one of its diagonal }\end{array}$ & - \\
\hline $\mathcal{T}_{j, 2}, j \in[1, J]$ & Either $D_{j, 2}$ or $D_{j, 2}^{\prime}$ according to the local splitting of $D_{j}$ & - \\
\hline $\mathcal{V}(i), i \in[1, I]$ & Set of integers $j \in[1, J]$ such that $A_{j}$ is a side of $T_{i}$ & - \\
\hline $\mathcal{E}(k), k \in[1, K]$ & Set of integers $j \in[1, J]$ such that $S_{k}$ is a node of $A_{j}$. & - \\
\hline$s_{j i}, i \in[1, I], j \in \mathcal{V}(i)$ & Equals +1 or -1 whether $\mathbf{n}_{j}$ points outwards or inwards $T_{i}$ & - \\
\hline $\mathbf{n}_{j i}, i \in[1, I], j \in \mathcal{V}(i)$ & Equals $s_{j i} \mathbf{n}_{j}=$ unit normal vector to $A_{j}$ pointing outwards $T_{i}$ & - \\
\hline$s_{j k}^{\prime}, k \in[1, K], j \in \mathcal{E}(k)$ & Equals +1 or -1 whether $\mathbf{n}_{j}^{\prime}$ points outwards or inwards $P_{k}$ & - \\
\hline $\mathbf{n}_{j k}^{\prime}, k \in[1, K], j \in \mathcal{E}(k)$ & Equals $s_{j k}^{\prime} \mathbf{n}_{j}^{\prime}=$ unit normal vector to $A_{j}^{\prime}$ pointing outwards $P_{k}$ & - \\
\hline$M_{j}, j \in[1, J]$ & The intersection of the diagonals of $D_{j}$ & Fig. 10 \\
\hline$D_{j, \alpha \beta},(\alpha, \beta) \in\{1 ; 2\}^{2}$ & The triangle $M_{j} G_{i_{\alpha}(j)} S_{k_{\beta}(j)}$ & Fig. 10 \\
\hline
\end{tabular}




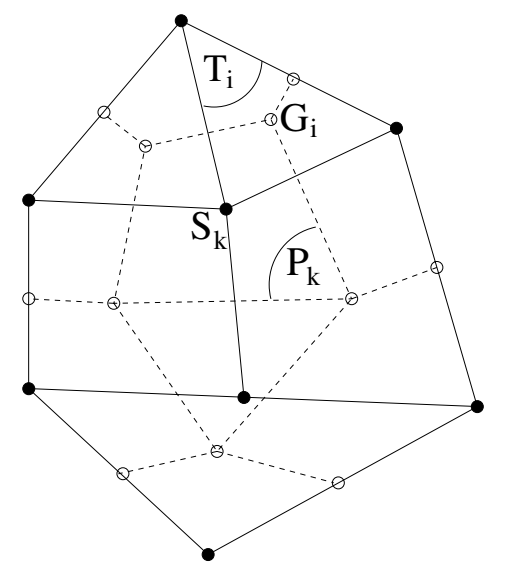

Figure 1. An example of primal and associated dual mesh.



Figure 2. The polygons associated to $S_{1}$ and $S_{2}$ overlap.

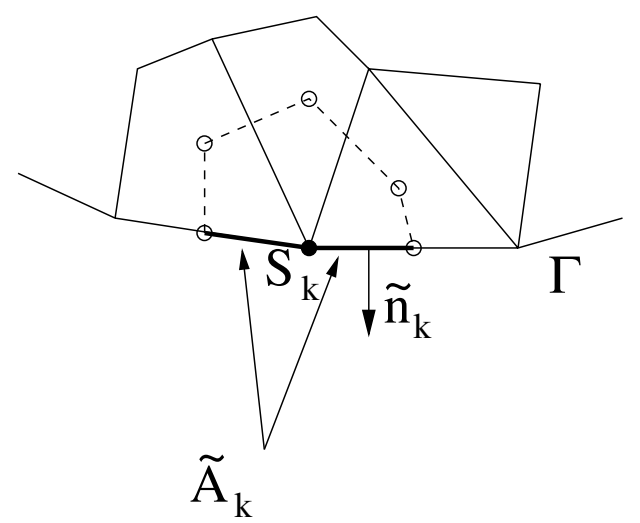

Figure 3. Definition of $\tilde{A}_{k}$ and $\tilde{\mathbf{n}}_{k}$ for the boundary nodes.

$G_{i_{1}(j)}$ associated to the only element of the primal mesh of which $A_{j}$ is a side and to the point $G_{i_{2}(j)}$ associated to $A_{j}$ (i.e. by convention $i_{2}(j)$ is element of $\left[I+1, I+J^{\Gamma}\right]$ when $A_{j}$ is located on $\Gamma$ ). The cells $D_{j}$ constitute a third partition of $\Omega$, which we name "diamond-mesh". The area of the cell $D_{j}$ is denoted by $\left|D_{j}\right|$. Examples of diamond-cells are displayed in Figure 4.

The following notations are summarized in Figure 5: We denote by $M_{i_{\alpha}(j) k_{\beta}(j)}$ the midpoint of the segment $\left[G_{i_{\alpha}(j)} S_{k_{\beta}(j)}\right]$, for each pair of integers $(\alpha, \beta)$ in $\{1 ; 2\}^{2}$. The unit vector normal to $A_{j}$ is denoted by $\mathbf{n}_{j}$ and is oriented so that $\mathbf{G}_{\mathbf{i}_{1}(\mathbf{j})} \mathbf{G}_{\mathbf{i}_{2}(\mathbf{j})} \cdot \mathbf{n}_{j} \geq 0$. We further denote by $A_{j}^{\prime}$ the segment $\left[G_{i_{1}(j)} G_{i_{2}(j)}\right]$ (whose length is $\left|A_{j}^{\prime}\right|$ ) and by $\mathbf{n}_{j}^{\prime}$ the unit vector normal to $A_{j}^{\prime}$ oriented so that $\mathbf{S}_{\mathbf{k}_{\mathbf{1}}(\mathbf{j})} \mathbf{S}_{\mathbf{k}_{\mathbf{2}}(\mathbf{j})} \cdot \mathbf{n}_{j}^{\prime} \geq 0$. 




(a) Inner cell

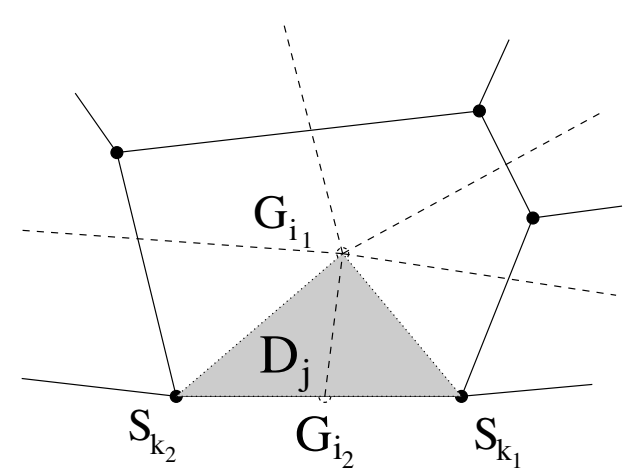

(b) Boundary cell

FigurE 4. Examples of diamond-cells.



FiguRE 5. Notations for the diamond-cell.

For $j \in[1, J]$, as indicated in Figure 6 , we also denote by $D_{j, 1}$ and $D_{j, 2}$, the triangles $S_{k_{1}(j)} G_{i_{1}(j)} S_{k_{2}(j)}$ and $\left.S_{k_{2}(j)} G_{i_{2}(j)} S_{k_{1}(j)}\right)$. In the same way, we denote by $D_{j, 1}^{\prime}$ and $D_{j, 2}^{\prime}$, the triangles $G_{i_{2}(j)} S_{k_{1}(j)} G_{i_{1}(j)}$ and $G_{i_{1}(j)} S_{k_{2}(j)} G_{i_{2}(j)}$.

Further, we define for each $i \in[1, I]$ the set $\mathcal{V}(i)$ of integers $j \in[1, J]$ such that $A_{j}$ is a side of $T_{i}$ and for each $k \in[1, K]$ the set $\mathcal{E}(k)$ of integers $j \in[1, J]$ such that $S_{k}$ is a node of $A_{j}$.

We define for each $j \in[1, J]$ and each $k$ such that $j \in \mathcal{E}(k)$ (resp. each $i$ such that $j \in \mathcal{V}(i)$ ) the real-valued number $s_{j k}^{\prime}$ (resp. $s_{j i}$ ) whose value is +1 or -1 whether $\mathbf{n}_{j}^{\prime}\left(\right.$ resp. $\left.\mathbf{n}_{j}\right)$ points outwards or inwards $P_{k}\left(\right.$ resp. $\left.T_{i}\right)$. We define $\mathbf{n}_{j k}^{\prime}:=s_{j k}^{\prime} \mathbf{n}_{j}^{\prime}$ (resp. $\quad \mathbf{n}_{j i}:=s_{j i} \mathbf{n}_{j}$ ) and remark that $\mathbf{n}_{j k}^{\prime}$ (resp. $\mathbf{n}_{j i}$ ) always points outwards $P_{k}$ $\left(\right.$ resp. $\left.T_{i}\right)$.

As will be seen in the following, we shall associate with each point $G_{i}\left(i \in\left[1, I+J^{\Gamma}\right]\right)$ and each vertex $S_{k}(k \in[1, K])$ discrete unknowns respectively denoted by $\phi_{i}^{T}$ and $\phi_{k}^{P}$. This leads us to the definition of the 




Figure 6. A diamond-cell may be split into two triangles in two distinct ways.

following discrete scalar product for all $(\phi, \psi)=\left(\left(\phi_{i}^{T}, \phi_{k}^{P}\right),\left(\psi_{i}^{T}, \psi_{k}^{P}\right)\right) \in\left(\mathbb{R}^{I} \times \mathbb{R}^{K}\right)^{2}$.

$$
(\phi, \psi)_{T, P}:=\frac{1}{2}\left(\sum_{i \in[1, I]}\left|T_{i}\right| \phi_{i}^{T} \psi_{i}^{T}+\sum_{k \in[1, K]}\left|P_{k}\right| \phi_{k}^{P} \psi_{k}^{P}\right) .
$$

In the same way, we define a discrete scalar product on the diamond mesh for all $(u, v)=\left(\left(u_{j}\right),\left(v_{j}\right)\right) \in\left(\mathbb{R}^{J}\right)^{2}$

$$
(u, v)_{D}:=\sum_{j \in[1, J]}\left|D_{j}\right| u_{j} v_{j}
$$

and a discrete scalar product for the traces of $u \in \mathbb{R}^{J}$ and $\phi \in \mathbb{R}^{I+J^{\Gamma}} \times \mathbb{R}^{K}$ on the boundary

$$
(u, \phi)_{\Gamma, h}:=\sum_{j \in\left[J-J^{\Gamma}+1, J\right]}\left|A_{j}\right| u_{j} \times \frac{1}{4}\left(\phi_{k_{1}(j)}^{P}+2 \phi_{i_{2}(j)}^{T}+\phi_{k_{2}(j)}^{P}\right) .
$$

Finally, for any $\phi \in \mathbb{R}^{I+J^{\Gamma}} \times \mathbb{R}^{K}$, we shall define a discrete $\mathrm{H}^{1}$ semi-norm on the diamond mesh with the help of the discrete gradient operator to be defined below (see Eq. (6)):

$$
|\phi|_{1, D}:=\left(\nabla_{h} \phi, \nabla_{h} \phi\right)_{D}^{1 / 2}
$$

We define the mesh step $h$ by

$$
h:=\sup _{i \in[1, I]} \operatorname{diam}\left(T_{i}\right)
$$

We shall denote by $\mathrm{L}^{p}(\Omega)$ the space of functions whose $p$-th power is integrable on $\Omega$, and by $\|\cdot\|_{\mathrm{L}^{p}, \Omega}$ the corresponding norm. Further, $\mathrm{H}^{m}$ is the space of functions $v$ of $\mathrm{L}^{2}(\Omega)$ whose partial derivatives (in the distributional sense) $\partial^{\alpha} v$, with $|\alpha| \leq m$ all belong to $\mathrm{L}^{2}(\Omega)$, while $\|\cdot\|_{m, \Omega}$ is the associated norm, and $|\cdot|_{m, \Omega}$ the associated semi-norm. Finally, the standard $\mathrm{L}^{2}(\Omega)$ inner product will be denoted by $(\cdot, \cdot)_{\Omega}$. 


\section{Construction of the Discrete Gradient And Divergence operators}

We define the discrete gradient of a function $\phi$ by its values on the diamond-cells of the mesh. We follow [6] and compute the mean-value of the gradient of any function $\phi$ on such a cell $D_{j}$ by the following formula:

$$
\left|D_{j}\right|\left\langle\nabla \phi_{\mid D_{j}}\right\rangle=\int_{D_{j}} \nabla \phi(\mathbf{x}) \mathrm{d} \mathbf{x}=\int_{\partial D_{j}} \phi(\xi) \mathbf{n}(\xi) \mathrm{d} \xi=\sum_{(\alpha, \beta) \in\{1 ; 2\}^{2}} \int_{\left[G_{i_{\alpha}} S_{k_{\beta}}\right]} \phi(\xi) \mathbf{n}_{G_{i_{\alpha}} S_{k_{\beta}}} \mathrm{d} \xi,
$$

where $\mathbf{n}(\xi)$ stands for the outward unit normal vector to $D_{j}$ at point $\xi$. Its constant value on each of the sides $\left[G_{i_{\alpha}} S_{k_{\beta}}\right]$ of $D_{j}$ is denoted by $\mathbf{n}_{G_{i_{\alpha}} S_{k_{\beta}}}$. The integrals in (5) can be approached by the following formula:

$$
\int_{[G S]} \phi(\xi) \mathrm{d} \xi \approx \ell_{G S} \frac{[\phi(G)+\phi(S)]}{2},
$$

where $\ell_{G S}$ denotes the length of the segment $[G S]$. Summing the contributions of the different nodes of $D_{j}$ and using elementary geometrical equalities allows us to give the definition of the discrete gradient $\nabla_{h}$ on $D_{j}$.

Definition 2.1. The discrete gradient $\nabla_{h}$ is defined by its values over the diamond-cells $D_{j}$ :

$$
\left(\nabla_{h} \phi\right)_{j}:=\frac{1}{2\left|D_{j}\right|}\left\{\left[\phi_{k_{2}}^{P}-\phi_{k_{1}}^{P}\right]\left|A_{j}^{\prime}\right| \mathbf{n}_{j}^{\prime}+\left[\phi_{i_{2}}^{T}-\phi_{i_{1}}^{T}\right]\left|A_{j}\right| \mathbf{n}_{j}\right\}
$$

where we have set $\phi_{k_{\alpha}}^{P}:=\phi\left(S_{k_{\alpha}}\right)$ and $\phi_{i_{\alpha}}^{T}:=\phi\left(G_{i_{\alpha}}\right)$, for $\alpha \in\{1 ; 2\}$.

Note that formula (6) is exact for affine functions. Computing the discrete gradient only requires the values of $\phi$ at the nodes of the primal and dual meshes. The operator $\nabla_{h}$ thus acts from $\mathbb{R}^{I+J^{\Gamma}} \times \mathbb{R}^{K}$ into $\left(\mathbb{R}^{J}\right)^{2}$.

Next, we choose to define the discrete divergence of a vector field $\mathbf{u}$ by its values both on the primal and dual cells of the mesh. A very natural way to do so on the primal cell $T_{i}$ is to write

$$
\left|T_{i}\right|\left\langle\nabla \cdot \mathbf{u}_{\mid T_{i}}\right\rangle=\int_{T_{i}} \nabla \cdot \mathbf{u}(\mathbf{x}) \mathrm{d} \mathbf{x}=\int_{\partial T_{i}} \mathbf{u}(\xi) \cdot \mathbf{n}(\xi)=\sum_{j \in \mathcal{V}(i)} \int_{A_{j}} \mathbf{u}(\xi) \cdot \mathbf{n}_{j i},
$$

where we recall that $\mathcal{V}(i)$ is the set of integers $j \in[1, J]$ such that $A_{j}$ is a side of $T_{i}$ and that $\mathbf{n}_{j i}$ is the unit vector orthogonal to $A_{j}$ pointing outward $T_{i}$. Supposing that the vector field $\mathbf{u}$ is given by its discrete values $\mathbf{u}_{j}$ on the cells $D_{j}$, and performing a similar computation over the cells $P_{k}$, we state the definition of the discrete divergence $\nabla_{h}^{T}$. on each $T_{i}$ and the discrete divergence $\nabla_{h}^{P}$. on each $P_{k}$.

Definition 2.2. The discrete divergence $\nabla_{h} \cdot:=\left(\nabla_{h}^{T} \cdot \nabla_{h}^{P} \cdot\right)$ is defined by its values over the primal cells $T_{i}$ and the dual cells $P_{k}$ :

$$
\begin{aligned}
\left(\nabla_{h}^{T} \cdot \mathbf{u}\right)_{i} & :=\frac{1}{\left|T_{i}\right|} \sum_{j \in \mathcal{V}(i)}\left|A_{j}\right| \mathbf{u}_{j} \cdot \mathbf{n}_{j i} \\
\left(\nabla_{h}^{P} \cdot \mathbf{u}\right)_{k} & :=\frac{1}{\left|P_{k}\right|}\left(\sum_{j \in \mathcal{E}(k)}\left|A_{j}^{\prime}\right| \mathbf{u}_{j} \cdot \mathbf{n}_{j k}^{\prime}+\sum_{j \in \mathcal{E}(k) \cap\left[J-J^{\Gamma}+1, J\right]} \frac{1}{2}\left|A_{j}\right| \mathbf{u}_{j} \cdot \mathbf{n}_{j}\right) .
\end{aligned}
$$

For a given function $\mathbf{u}$, it is straightforward to check that these formulæ are the exact mean-values of $\nabla \cdot \mathbf{u}$ over $T_{i}$ (respectively over an inner $P_{k}$ ) if $\mathbf{u}_{j} \cdot \mathbf{n}_{j i}\left(\right.$ resp. $\left.\mathbf{u}_{j} \cdot \mathbf{n}_{j k}^{\prime}\right)$ represents the mean-value of $\mathbf{u} \cdot \mathbf{n}_{j i}$ over $A_{j}($ resp. of $\mathbf{u} \cdot \mathbf{n}_{j k}^{\prime}$ over $\left.A_{j}^{\prime}\right)$. The operator $\nabla_{h}$ acts from $\left(\mathbb{R}^{J}\right)^{2}$ into $\mathbb{R}^{I} \times \mathbb{R}^{K}$. 
Proposition 2.3. The following discrete analogue of the Green formula holds:

$$
\left(\nabla_{h} \cdot \mathbf{u}, \phi\right)_{T, P}=-\left(\mathbf{u}, \nabla_{h} \phi\right)_{D}+(\mathbf{u} \cdot \mathbf{n}, \phi)_{\Gamma, h}
$$

for all $\mathbf{u} \in\left(\mathbb{R}^{J}\right)^{2}$ and all $\phi=\left(\phi^{T}, \phi^{P}\right) \in \mathbb{R}^{I+J^{\Gamma}} \times \mathbb{R}^{K}$, where the definitions (2), (3) and (4) have been used.

Proof. Let $\mathbf{u}$ be a discrete vector field and $\nabla_{h} \phi$ the discrete gradient of a function $\phi$, respectively defined by the values $\mathbf{u}_{j}$ and formula (6) on each of the diamond-cells $D_{j}$. There holds:

$$
\left(\mathbf{u}, \nabla_{h} \phi\right)_{D}=\sum_{j \in[1, J]}\left|D_{j}\right| \mathbf{u}_{j} \cdot\left(\nabla_{h} \phi\right)_{j}=\sum_{j \in[1, J]} \frac{1}{2} \mathbf{u}_{j} \cdot\left[\left(\phi_{k_{2}(j)}^{P}-\phi_{k_{1}(j)}^{P}\right)\left|A_{j}^{\prime}\right| \mathbf{n}_{j}^{\prime}+\left(\phi_{i_{2}(j)}^{T}-\phi_{i_{1}(j)}^{T}\right)\left|A_{j}\right| \mathbf{n}_{j}\right]
$$

For a given $k \in[1, K]$, we sum in (9) the different contributions of $\phi_{k}^{P}$, taking into account the orientation of the vectors $\mathbf{n}_{j}^{\prime}$. We recall the definition of $s_{j k}^{\prime}$ to be either +1 or -1 whether $\mathbf{n}_{j}^{\prime}$ points outwards or inwards $P_{k}$. In $(9)$, the term $\phi_{k}^{P}$ appears each time that $S_{k}$ belongs to a certain $A_{j}$ and this term is multiplied by $-\frac{1}{2} s_{j k}^{\prime}\left|A_{j}^{\prime}\right| \mathbf{u}_{j} \cdot \mathbf{n}_{j}^{\prime}$. Indeed, if for this given $j$ there holds $k=k_{1}(j)$, then $\mathbf{n}_{j}^{\prime}$ points outwards $P_{k}$ (see Fig. 5), and if there holds $k=k_{2}(j)$, then $\mathbf{n}_{j}^{\prime}$ points inwards $P_{k}$. In the same way, for $i \in[1, I]$, the term $\phi_{i}^{T}$ appears each time that $G_{i}$ belongs to a certain $A_{j}^{\prime}$ (i.e. each time that $A_{j}$ is a side of $T_{i}$ ) and is multiplied by $-\frac{1}{2} s_{j i}\left|A_{j}\right| \mathbf{u}_{j} \cdot \mathbf{n}_{j}$. Finally, for $i \in\left[I+1, I+J^{\Gamma}\right]$, the term $\phi_{i}^{T}$ appears only once, namely for $j(i)$, and by convention $i=i_{2}(j(i))$, so that $\phi_{i}^{T}$ is multiplied by $\frac{1}{2}\left|A_{j(i)}\right| \mathbf{u}_{j(i)} \cdot \mathbf{n}_{j(i)}$. As a result, we can rewrite (9) in the following way:

$$
\begin{aligned}
\left(\mathbf{u}, \nabla_{h} \phi\right)_{D}= & -\frac{1}{2} \sum_{k \in[1, K]} \phi_{k}^{P}\left(\sum_{j \in \mathcal{E}(k)}\left|A_{j}^{\prime}\right| \mathbf{u}_{j} \cdot s_{j k}^{\prime} \mathbf{n}_{j}^{\prime}\right) \\
& -\frac{1}{2} \sum_{i \in[1, I]} \phi_{i}^{T}\left(\sum_{j \in \mathcal{V}(i)}\left|A_{j}\right| \mathbf{u}_{j} \cdot s_{j i} \mathbf{n}_{j}\right)+\frac{1}{2} \sum_{i \in\left[I+1, I+J^{\Gamma}\right]} \phi\left(G_{i}\right)\left|A_{j(i)}\right| \mathbf{u}_{j(i)} \cdot \mathbf{n}_{j(i)} .
\end{aligned}
$$

We wish to compute $-\left(\mathbf{u}, \nabla_{h} \phi\right)_{D}+(\mathbf{u} \cdot \mathbf{n}, \phi)_{\Gamma, h}$, where the boundary part is given by (4). In this sum, the contribution of $\phi_{i}^{T}$ for $i \in\left[I+1, I+J^{\Gamma}\right]$ in $(\mathbf{u} \cdot \mathbf{n}, \phi)_{\Gamma, h}$ exactly cancels that in (10). Moreover, for $k \in\left[K-J^{\Gamma}+1, K\right]$, the term $\phi_{k}^{P}$ appears twice in $(\mathbf{u} \cdot \mathbf{n}, \phi)_{\Gamma, h}$, namely once for each $j$ such that $A_{j}$ belongs to $\Gamma$ and such that $S_{k}$ belongs to $A_{j}$. This implies that

$$
\begin{aligned}
-\left(\mathbf{u}, \nabla_{h} \phi\right)_{D}+(\mathbf{u} \cdot \mathbf{n}, \phi)_{\Gamma}= & \frac{1}{2} \sum_{k \in[1, K]} \phi_{k}^{P}\left(\sum_{j \in \mathcal{E}(k)}\left|A_{j}^{\prime}\right| \mathbf{u}_{j} \cdot \mathbf{n}_{j k}^{\prime}\right) \\
& +\frac{1}{2} \sum_{k \in\left[K-J^{\Gamma}+1, K\right]} \phi_{k}^{P} \sum_{j \in \mathcal{E}(k) \cap\left[J-J^{\Gamma}+1, J\right]} \frac{1}{2}\left|A_{j}\right| \mathbf{u}_{j} \cdot \mathbf{n}_{j} \\
& +\frac{1}{2} \sum_{i \in[1, I]} \phi_{i}^{T}\left(\sum_{j \in \mathcal{V}(i)}\left|A_{j}\right| \mathbf{u}_{j} \cdot \mathbf{n}_{j i}\right)
\end{aligned}
$$

which is exactly $\left(\nabla_{h} \cdot \mathbf{u}, \phi\right)_{T, P}$ according to $(2)$ and $(7)$. 


\section{The Finite VOlume SCHEME}

In this part, we use the above-defined discrete divergence and gradient operators to construct the finite volume scheme in order to compute $\phi:=\left(\left(\phi_{i}^{T}\right),\left(\phi_{k}^{P}\right)\right) \in \mathbb{R}^{I+J^{\Gamma}} \times \mathbb{R}^{K}$ that would be a good approximation of the exact solution $\hat{\phi}$ of Equation (1). Paragraphs 3.1 and subsequent are devoted to homogeneous Dirichlet boundary conditions, while Section 3.4 considers the case of homogeneous Neumann boundary conditions.

\subsection{Equations to be solved - The case of Dirichlet boundary conditions}

On each primal cell $T_{i}, i \in[1, I]$, and on each inner dual cell $P_{k}$, with $k \in\left[1, K-J^{\Gamma}\right]$, the equation $-\Delta \phi=f$ is approximated by using the above-defined discrete divergence and gradient operators. We write:

$$
\begin{aligned}
-\left(\nabla_{h}^{T} \cdot\left(\nabla_{h} \phi\right)\right)_{i} & =f_{i}^{T} \forall i \in[1, I] \\
-\left(\nabla_{h}^{P} \cdot\left(\nabla_{h} \phi\right)\right)_{k} & =f_{k}^{P} \quad \forall k \in\left[1, K-J^{\Gamma}\right]
\end{aligned}
$$

where $f_{i}^{T}$ and $f_{k}^{P}$ are the mean-values of $f$ over $T_{i}$ and $P_{k}$ defined by:

$$
f_{i}^{T}=\frac{1}{\left|T_{i}\right|} \int_{T_{i}} f(\mathbf{x}) \mathrm{d} \mathbf{x} \text { and } f_{k}^{P}=\frac{1}{\left|P_{k}\right|} \int_{P_{k}} f(\mathbf{x}) \mathrm{d} \mathbf{x}
$$

Homogeneous Dirichlet boundary conditions are discretized by:

$$
\phi_{i}^{T}=0 \forall i \in\left[I+1, I+J^{\Gamma}\right] \text { and } \phi_{k}^{P}=0 \forall k \in\left[K-J^{\Gamma}+1, K\right] .
$$

Thus, we seek $\phi$ in the set $V$ defined by:

$$
\begin{aligned}
V:=\{\phi= & \left(\left(\phi_{i}^{T}\right),\left(\phi_{k}^{P}\right)\right) \in \mathbb{R}^{I+J^{\Gamma}} \times \mathbb{R}^{K} / \quad \text { s.t. } \\
& \left.\phi_{i}^{T}=0 \forall i \in\left[I+1, I+J^{\Gamma}\right] \quad \text { and } \quad \phi_{k}^{P}=0 \quad \forall k \in\left[K-J^{\Gamma}+1, K\right]\right\} .
\end{aligned}
$$

The linear system has $I+J^{\Gamma}+K$ unknowns and as many equations given by (11), (12) and (14).

Remark 3.1. If, for each diamond-cell $D_{j}$, the segments $A_{j}$ and $A_{j}^{\prime}$ are orthogonal (e.g. in the case of Voronoi meshes or "admissible meshes" in the sense of [9]), the scheme decouples into two disjoint subsystems and reduces to the usual schemes presented in [9]. Indeed, as $\mathbf{n}_{j} \cdot \mathbf{n}_{j}^{\prime}=0$ and $\left|D_{j}\right|=\frac{\left|A_{j}\right|\left|A_{j}^{\prime}\right|}{2}$, then $\left(\nabla_{h} \phi\right)_{j} \cdot \mathbf{n}_{j i}=\frac{\phi_{i j}^{T}-\phi_{i}^{T}}{\left|A_{j}^{\prime}\right|}$ (where $\phi_{i j}^{T}$ is the unknown associated to the neighbouring primal cell), which is simply a finite difference evaluation of the normal component of the gradient on $A_{j}$.

\subsection{Symmetry, existence and uniqueness}

Proposition 3.2. The matrix associated to the linear system (11), (12) and (14) is symmetrical with respect to the scalar product $(\cdot, \cdot)_{T, P}$. The scheme possesses a unique solution.

Proof. For all $(\phi, \psi) \in V^{2}$, applying formula (8) twice proves the symmetry:

$$
-\left(\nabla_{h} \cdot\left(\nabla_{h} \phi\right), \psi\right)_{T, P}=\left(\nabla_{h} \phi, \nabla_{h} \psi\right)_{D}=-\left(\phi, \nabla_{h} \cdot\left(\nabla_{h} \psi\right)\right)_{T, P}
$$

Further, existence and uniqueness are equivalent for this square linear system. Choosing $f_{i}^{T}=f_{k}^{P}=0$, we have:

$$
-\left(\nabla_{h}^{T} \cdot\left(\nabla_{h} \phi\right)\right)_{i}=0 \forall i \in[1, I] \text { and }-\left(\nabla_{h}^{P} \cdot\left(\nabla_{h} \phi\right)\right)_{k}=0 \forall k \in\left[1, K-J^{\Gamma}\right]
$$


as well as

$$
\phi_{k}^{P}=0 \forall k \in\left[K-J^{\Gamma}+1, K\right] .
$$

Thus,

$$
-\left(\nabla_{h}^{T} \cdot\left(\nabla_{h} \phi\right)\right)_{i} \phi_{i}^{T}=0 \forall i \in[1, I] \text { and }-\left(\nabla_{h}^{P} \cdot\left(\nabla_{h} \phi\right)\right)_{k} \phi_{k}^{P}=0 \forall k \in[1, K] .
$$

As a consequence, there holds:

$$
-\left(\nabla_{h} \cdot\left(\nabla_{h} \phi\right), \phi\right)_{T, P}=\left(\nabla_{h} \phi, \nabla_{h} \phi\right)_{D}=\sum_{j \in[1, J]}\left|D_{j}\right|\left|\left(\nabla_{h} \phi\right)_{j}\right|^{2}=0
$$

which implies that $\left(\nabla_{h} \phi\right)_{j}$ vanishes for all $j$. Consequently, there exists two constants $c^{T}$ and $c^{P}$ such that:

$$
\forall i \in\left[1, I+J^{\Gamma}\right] \phi_{i}^{T}=c^{T} \text { and } \forall k \in[1, K] \phi_{k}^{P}=c^{P} .
$$

Taking into account the boundary conditions (14), we have $c^{T}=c^{P}=0$. Therefore, uniqueness, and thus existence, of the solution of the system (11), (12), (14) is proved.

\subsection{Interpretation under a variational formulation}

Let us start by the following definition

Definition 3.3. Let $\hat{\phi}$ be the exact solution of Equation (1), together with homogeneous Dirichlet boundary conditions. On each diamond-cell $D_{j}$, we define the constant vector $(\delta \hat{\phi})_{j}$ by the following scalar products:

$$
(\delta \hat{\phi})_{j} \cdot \mathbf{n}_{j}=\frac{1}{\left|A_{j}\right|} \int_{A_{j}} \nabla \hat{\phi} \cdot \mathbf{n}_{j} \mathrm{~d} \xi \text { and }(\delta \hat{\phi})_{j} \cdot \mathbf{n}_{j}^{\prime}=\frac{1}{\left|A_{j}^{\prime}\right|} \int_{A_{j}^{\prime}} \nabla \hat{\phi} \cdot \mathbf{n}_{j}^{\prime} \mathrm{d} \xi .
$$

We can prove the following proposition:

Proposition 3.4. The solution $\phi \in V$ of the system (11), (12), (14) is such that $\forall \psi \in V$,

$$
\left(\nabla_{h} \phi, \nabla_{h} \psi\right)_{D}=\left(\delta \hat{\phi}, \nabla_{h} \psi\right)_{D}
$$

Proof. Let $\psi=\left(\left(\psi_{i}^{T}\right),\left(\psi_{k}^{P}\right)\right) \in V$. Let us denote by $\psi_{h}^{*}$ the following function:

$$
\psi_{h}^{*}(\mathbf{x}):=\frac{1}{2}\left(\sum_{i \in[1, I]} \psi_{i}^{T} \theta_{i}^{T}(\mathbf{x})+\sum_{k \in[1, K]} \psi_{k}^{P} \theta_{k}^{P}(\mathbf{x})\right) .
$$

We have

But we can write

$$
\left(\Delta \hat{\phi}, \psi_{h}^{*}\right)_{\Omega}=\frac{1}{2}\left(\sum_{i \in[1, I]} \int_{T_{i}} \Delta \hat{\phi} \mathrm{d} \mathbf{x} \psi_{i}^{T}+\sum_{k \in[1, K]} \int_{P_{k}} \Delta \hat{\phi} \mathrm{d} \mathbf{x} \psi_{k}^{P}\right)
$$

$$
\sum_{i \in[1, I]} \int_{T_{i}} \Delta \hat{\phi} \mathrm{d} \mathbf{x} \psi_{i}^{T}=\sum_{i \in[1, I]}\left(\sum_{j \in \mathcal{V}(i)} \int_{A_{j}} \nabla \hat{\phi} \cdot \mathbf{n}_{j i} \mathrm{~d} \xi\right) \psi_{i}^{T} .
$$

As $\psi_{i}^{T}=0 \forall i \in\left[I+1, I+J^{\Gamma}\right]$, Equation (17) can also be written

$$
\sum_{i \in[1, I]} \int_{T_{i}} \Delta \hat{\phi} \mathrm{d} \mathbf{x} \psi_{i}^{T}=\sum_{i \in[1, I]}\left(\sum_{j \in \mathcal{V}(i)} \int_{A_{j}} \nabla \hat{\phi} \cdot \mathbf{n}_{j i} \mathrm{~d} \xi\right) \psi_{i}^{T}-\sum_{i \in\left[I+1, I+J^{\Gamma}\right]} \int_{A_{j(i)}} \nabla \hat{\phi} \cdot \mathbf{n}_{j(i)} \mathrm{d} \xi \psi_{i}^{T} .
$$


Moreover, recalling that $\tilde{A}_{k}$ is the intersection of $\Gamma$ and $\partial P_{k}$ for the boundary dual cell $P_{k}$ and $\tilde{\mathbf{n}}_{k}$ the outgoing normal unit vector on $\tilde{A}_{k}$, there holds

$$
\begin{aligned}
\sum_{k \in[1, K]} \int_{P_{k}} \Delta \hat{\phi} \mathrm{d} \mathbf{x} \psi_{k}^{P} & =\sum_{k \in[1, K]}\left(\sum_{j \in \mathcal{E}(k)} \int_{A_{j}^{\prime}} \nabla \hat{\phi} \cdot \mathbf{n}_{j k}^{\prime} \mathrm{d} \xi\right) \psi_{k}^{P}+\sum_{k \in\left[K-J^{\Gamma}+1, K\right]} \int_{\tilde{A}_{k}} \nabla \hat{\phi} \cdot \tilde{\mathbf{n}}_{k} \mathrm{~d} \xi \psi_{k}^{P} \\
& =\sum_{k \in[1, K]}\left(\sum_{j \in \mathcal{E}(k)} \int_{A_{j}^{\prime}} \nabla \hat{\phi} \cdot \mathbf{n}_{j k}^{\prime} \mathrm{d} \xi\right) \psi_{k}^{P}
\end{aligned}
$$

because $\psi_{k}^{P}=0$ when $k \in\left[K-J^{\Gamma}+1, K\right]$.

Then, summing (18) and (19) and rewriting the sums over $i$ and $k$ into a sum over $j$, we obtain:

$$
-\left(\Delta \hat{\phi}, \psi_{h}^{*}\right)_{\Omega}=\sum_{j \in[1, J]}-\frac{1}{2}\left(\int_{A_{j}} \nabla \hat{\phi} \cdot \mathbf{n}_{j} \mathrm{~d} \xi\left(\psi_{i_{1}(j)}^{T}-\psi_{i_{2}(j)}^{T}\right)+\int_{A_{j}^{\prime}} \nabla \hat{\phi} \cdot \mathbf{n}_{j}^{\prime} \mathrm{d} \xi\left(\psi_{k_{1}(j)}^{P}-\psi_{k_{2}(j)}^{P}\right)\right) .
$$

Because of Definitions 2.1 and 3.3, we have the following equality:

$$
\begin{aligned}
(\delta \hat{\phi})_{j} \cdot\left(\nabla_{h} \psi\right)_{j} & =-\frac{1}{2\left|D_{j}\right|}\left(\psi_{k_{1}(j)}^{P}-\psi_{k_{2}(j)}^{P}\right)\left|A_{j}^{\prime}\right|(\delta \hat{\phi})_{j} \cdot \mathbf{n}_{j}^{\prime}-\frac{1}{2\left|D_{j}\right|}\left(\psi_{i_{1}(j)}^{T}-\psi_{i_{2}(j)}^{T}\right)\left|A_{j}\right|(\delta \hat{\phi})_{j} \cdot \mathbf{n}_{j} \\
& =-\frac{1}{2\left|D_{j}\right|}\left[\left(\psi_{k_{1}(j)}^{P}-\psi_{k_{2}(j)}^{P}\right) \int_{A_{j}^{\prime}} \nabla \hat{\phi} \cdot \mathbf{n}_{j}^{\prime} \mathrm{d} \xi+\left(\psi_{i_{1}(j)}^{T}-\psi_{i_{2}(j)}^{T}\right) \int_{A_{j}} \nabla \hat{\phi} \cdot \mathbf{n}_{j} \mathrm{~d} \xi\right]
\end{aligned}
$$

and thus,

$$
-\left(\Delta \hat{\phi}, \psi_{h}^{*}\right)_{\Omega}=\sum_{j \in[1, J]}\left|D_{j}\right|(\delta \hat{\phi})_{j} \cdot\left(\nabla_{h} \psi\right)_{j}=\left(\delta \hat{\phi}, \nabla_{h} \psi\right)_{D}
$$

But on the other hand, as $-\Delta \hat{\phi}=f$, and with the definition (13),

$$
\begin{aligned}
-\left(\Delta \hat{\phi}, \psi_{h}^{*}\right)_{\Omega} & =\frac{1}{2}\left(\sum_{i \in[1, I]} \int_{T_{i}} f(\mathbf{x}) \mathrm{d} \mathbf{x} \psi_{i}^{T}+\sum_{k \in[1, K]} \int_{P_{k}} f(\mathbf{x}) \mathrm{d} \mathbf{x} \psi_{k}^{P}\right) \\
& =\frac{1}{2}\left(\sum_{i \in[1, I]}\left|T_{i}\right| f_{i}^{T} \psi_{i}^{T}+\sum_{k \in[1, K]}\left|P_{k}\right| f_{k}^{P} \psi_{k}^{P}\right) .
\end{aligned}
$$

Taking into account the scheme defined by (11)-(12), we have the following equality, for all $\psi \in V$ :

$$
-\left(\Delta \hat{\phi}, \psi_{h}^{*}\right)_{\Omega}=-\left(\nabla_{h} \cdot\left(\nabla_{h} \phi\right), \psi\right)_{T, P}=\left(\nabla_{h} \phi, \nabla_{h} \psi\right)_{D}
$$

which, thanks to (20), ends the proof. Then, $\phi$ is the element in $V$ which minimizes $\left(\nabla_{h} \psi-\delta \hat{\phi}, \nabla_{h} \psi-\delta \hat{\phi}\right)_{D}$. 


\subsection{The case of Neumann boundary conditions}

In this section, we consider Equation (1) supplemented with homogeneous Neumann boundary conditions:

$$
\nabla \phi \cdot \mathbf{n}=0,
$$

where $\mathbf{n}$ is the exterior unit normal vector on $\Gamma$. Note that the mean value of the right-hand side $f$ of $(1)$ is required to vanish:

$$
\int_{\Omega} f(\mathbf{x}) \mathrm{d} \mathbf{x}=0 .
$$

Additionally, $\phi$ is defined up to a constant which may be imposed through

$$
\int_{\Omega} \phi(\mathbf{x}) \mathrm{d} \mathbf{x}=0 .
$$

Equation (21) is approximated on each boundary side with the help of the discrete gradient operator. We write:

$$
\left(\nabla_{h} \phi\right)_{j} \cdot \mathbf{n}_{j}=0 \quad \forall j \in\left[J-J^{\Gamma}+1, J\right] .
$$

Thus, Equation (24) provides for $J^{\Gamma}$ boundary conditions, while Equation (14) provides for $2 J^{\Gamma}$ boundary conditions. Consequently, we extend Equation (12) to all $k \in[1, K]$. We thus write:

$$
\begin{aligned}
-\left(\nabla_{h}^{T} \cdot\left(\nabla_{h} \phi\right)\right)_{i} & =f_{i}^{T} \quad \forall i \in[1, I] \\
-\left(\nabla_{h}^{P} \cdot\left(\nabla_{h} \phi\right)\right)_{k} & =f_{k}^{P} \quad \forall k \in[1, K] .
\end{aligned}
$$

Finally, the following two equations

$$
\sum_{i \in[1, I]}\left|T_{i}\right| \phi_{i}^{T}=\sum_{k \in[1, K]}\left|P_{k}\right| \phi_{k}^{P}=0
$$

are obvious discrete versions of (23). Now the set (24), (25), (26) and (27) provides for $I+K+J^{\Gamma}+2$ equations, while there are only $I+K+J^{\Gamma}$ unknowns. But this is no matter, since we may prove the following proposition Proposition 3.5. Equations (24), (25) and (26) provide only for $I+K+J^{\Gamma}-2$ independent equations and the set (24), (25), (26) and (27) has a unique solution.

Proof. Multiplying (25) by $\left|T_{i}\right|$ and summing over $i \in[1, I]$ leads to

$$
-\sum_{i \in[1, I]}\left|T_{i}\right|\left(\nabla_{h}^{T} \cdot\left(\nabla_{h} \phi\right)\right)_{i}=\sum_{i \in[1, I]}\left|T_{i}\right| f_{i}^{T} .
$$

Defining $\psi:=\left(\left(\psi_{i}^{T}\right),\left(\psi_{k}^{P}\right)\right)$ such that $\psi_{i}^{T}=1 \quad \forall i \in\left[1, I+J^{\Gamma}\right]$ and $\psi_{k}^{P}=0 \quad \forall k \in[1, K]$, the left-hand side of (28) is equal to $-2\left(\nabla_{h} \cdot\left(\nabla_{h} \phi\right), \psi\right)_{T, P}$ (see Eq. (2)). Thanks to (6), there holds $\nabla_{h} \psi=0$, and thus, thanks to the discrete Green formula (8), the left-hand side of (28) is equal to

$$
\sum_{j \in\left[J-J^{\Gamma}+1, J\right]}\left|A_{j}\right|\left(\nabla_{h} \phi\right)_{j} \cdot \mathbf{n}_{j} .
$$

Thanks to boundary conditions (24), the left-hand side of (28) thus vanishes. On the other hand, the right-hand side of (28) can be evaluated thanks to (13) and is equal to

$$
\sum_{i \in[1, I]} \int_{T_{i}} f(\mathbf{x}) \mathrm{d} \mathbf{x}=\int_{\Omega} f(\mathbf{x}) \mathrm{d} \mathbf{x} .
$$


Thus thanks to (22), the right-hand side of (28) also vanishes and thus the set (24) and (25) is redundant. In the same way, multiplying (26) by $\left|P_{k}\right|$ and summing over $k \in[1, K]$ leads to the conclusion that the set (24) and (26) is redundant. Now in the set (24)-(27), there are as many independent equations as unknowns, thus we only have to prove injectivity. Setting $f_{i}^{T}=f_{k}^{P}=0$, multiplying (25) by $\left|T_{i}\right| \phi_{i}^{T}$, (26) by $\left|P_{k}\right| \phi_{k}^{P}$ and summing over $i \in[1, I]$ and $k \in[1, K]$ leads to

$$
-\left(\nabla_{h} \cdot\left(\nabla_{h} \phi\right), \phi\right)_{T, P}=\left(\nabla_{h} \phi, \nabla_{h} \phi\right)_{D}=\sum_{j \in[1, J]}\left|D_{j}\right|\left|\left(\nabla_{h} \phi\right)_{j}\right|^{2}=0
$$

by application of Equation (8) and taking into account boundary conditions (24). Equation (29) implies that $\left(\nabla_{h} \phi\right)_{j}$ vanishes for all $j$. Consequently, there exists two constants $c^{T}$ and $c^{P}$ such that:

$$
\forall i \in\left[1, I+J^{\Gamma}\right] \phi_{i}^{T}=c^{T} \text { and } \forall k \in[1, K] \phi_{k}^{P}=c^{P}
$$

Taking into account Equation (27), we have $c^{T}=c^{P}=0$, which completes the proof.

Remark 3.6. In practice, it may very well happen that

$$
\sum_{i \in[1, I]}\left|T_{i}\right| f_{i}^{T} \neq 0 \quad \text { and } / \text { or } \quad \sum_{k \in[1, K]}\left|P_{k}\right| f_{k}^{P} \neq 0
$$

This may be the case for example when $f_{i}$ and/or $f_{k}$ are not computed exactly, but rather by means of a quadrature formula. In this case, in order to maintain the solubility of the linear system, we have to project $\left(\left(f_{i}^{T}\right),\left(f_{k}^{P}\right)\right)$ onto the adequate subspace by defining a new right-hand side $\left(\left(\tilde{f}_{i}^{T}\right),\left(\tilde{f}_{k}^{P}\right)\right)$ through

$$
\tilde{f}_{i}^{T}=f_{i}^{T}-\frac{\sum_{i \in[1, I]}\left|T_{i}\right| f_{i}^{T}}{|\Omega|} \quad \text { and } \quad \tilde{f}_{k}^{P}=f_{k}^{P}-\frac{\sum_{k \in[1, K]}\left|P_{k}\right| f_{k}^{P}}{|\Omega|} .
$$

\section{Equivalence With a Finite Element Method}

The variational formulation obtained at the end of Section 3.3 induces us to look for a finite element space, the functions of which have a piecewise-constant gradient on each diamond-cell, equal to the discrete gradient of a certain element of $V$. We start by the following result:

Proposition 4.1. Given an element $\phi \in V$, there exists a unique function $\phi_{h}$ defined by

$$
\begin{array}{cc}
\left(\phi_{h}\right)_{\mid D_{j}} \in P^{1}\left(D_{j}\right) & \forall j \in[1, J] \\
\phi_{h}\left(M_{i_{\alpha}(j) k_{\beta}(j)}\right)=\frac{1}{2}\left(\phi_{i_{\alpha}(j)}^{T}+\phi_{k_{\beta}(j)}^{P}\right) & \forall j \in[1, J] \forall(\alpha, \beta) \in\{1 ; 2\}^{2} .
\end{array}
$$

Moreover, we have the following essential property:

$$
\left(\nabla \phi_{h}\right)_{\mid D_{j}}=\left(\nabla_{h} \phi\right)_{j}
$$

Proof. In the cell $D_{j}$, one can always find three $M_{i_{\alpha} k_{\beta}}$, with $(\alpha, \beta) \in\{1 ; 2\}^{2}$ that are not aligned. Let us suppose, without loss of generality, that they correspond to $(\alpha, \beta)$ in $\{(1,1),(1,2),(2,1)\}$. Then, there exists a unique function, still denoted by $\phi_{h}$, defined by (30) and $(31)$ applied to all $(\alpha, \beta)$ in $\{(1,1),(1,2),(2,1)\}$. Then, because $\phi_{h}$ is a first-order polynomial, we may write:

$$
\phi_{h}\left(M_{i_{2} k_{2}}\right)=\phi_{h}\left(M_{i_{2} k_{1}}\right)+\mathbf{M}_{\mathbf{i}_{\mathbf{2}} \mathbf{k}_{\mathbf{1}}} \mathbf{M}_{\mathbf{i}_{\mathbf{2}} \mathbf{k}_{\mathbf{2}}} \cdot \nabla \phi_{h} \quad \text { and } \quad \phi_{h}\left(M_{i_{1} k_{2}}\right)=\phi_{h}\left(M_{i_{1} k_{1}}\right)+\mathbf{M}_{\mathbf{i}_{\mathbf{1}} \mathbf{k}_{\mathbf{1}}} \mathbf{M}_{\mathbf{i}_{\mathbf{1}} \mathbf{k}_{\mathbf{2}}} \cdot \nabla \phi_{h}
$$


As $\left(M_{i_{1} k_{1}} M_{i_{1} k_{2}} M_{i_{2} k_{2}} M_{i_{2} k_{1}}\right)$ is a parallelogram, the equality $\mathbf{M}_{\mathbf{i}_{\mathbf{2}} \mathbf{k}_{\mathbf{1}}} \mathbf{M}_{\mathbf{i}_{\mathbf{2}} \mathbf{k}_{\mathbf{2}}}=\mathbf{M}_{\mathbf{i}_{\mathbf{1}} \mathbf{k}_{\mathbf{1}}} \mathbf{M}_{\mathbf{i}_{\mathbf{1}} \mathbf{k}_{\mathbf{2}}}$ holds and thus

$$
\phi_{h}\left(M_{i_{2} k_{2}}\right)=\phi_{h}\left(M_{i_{1} k_{2}}\right)+\phi_{h}\left(M_{i_{2} k_{1}}\right)-\phi_{h}\left(M_{i_{1} k_{1}}\right) .
$$

But with the definitions (31), this value is exactly $\frac{1}{2}\left(\phi_{i_{2}(j)}^{T}+\phi_{k_{2}(j)}^{P}\right)$, which ensures the existence of $\phi_{h}$ defined by (30) and (31) applied to all $(\alpha, \beta)$ in $\{1 ; 2\}^{2}$.

Moreover, as $\phi_{h} \in V_{h}$ is $P^{1}$ over $D_{j}$, its gradient is a constant. Then,

$$
\left(\nabla \phi_{h}\right)_{\mid D_{j}}=\frac{1}{\left|D_{j}\right|} \int_{D_{j}} \nabla \phi_{h} \mathrm{~d} \mathbf{x}
$$

By Green's formula, this quantity is also equal to

$$
\left(\nabla \phi_{h}\right)_{\mid D_{j}}=\frac{1}{\left|D_{j}\right|} \int_{\partial D_{j}} \phi_{h}(\xi) \mathbf{n}(\xi) \mathrm{d} \xi
$$

But $\partial D_{j}$ is composed of the four segments $\left[G_{i_{\alpha}} S_{k_{\beta}}\right]$, on which the restriction of $\phi_{h}$ is also $P_{1}$. Thus the four integrals in (33) can be evaluated exactly by the midpoint rule. The midpoint of $\left[G_{i_{\alpha}} S_{k_{\beta}}\right]$ is by definition $M_{i_{\alpha} k_{\beta}}$, where the value of $\phi_{h}$ is given by (31). Thus, we can sum the contributions of $\phi_{i_{\alpha}}^{T}$ and $\phi_{k_{\beta}}^{P}$ just like in Section 2 to obtain

$$
\left(\nabla \phi_{h}\right)_{\mid D_{j}}=\frac{1}{2\left|D_{j}\right|}\left[\left(\phi_{k_{2}}^{P}-\phi_{k_{1}}^{P}\right)\left|A_{j}^{\prime}\right| \mathbf{n}_{j}^{\prime}+\left(\phi_{i_{2}}^{T}-\phi_{i_{1}}^{T}\right)\left|A_{j}\right| \mathbf{n}_{j}\right]
$$

which is exactly $\left(\nabla_{h} \phi\right)_{j}$.

Definition 4.2. We define an operator $L$ on $V$ by associating to any $\phi \in V$ the function $\phi_{h}$ defined in Proposition 4.1: $\phi_{h}=L(\phi)$. Moreover, we set $V_{h}:=L(V)$. We note that $V_{h}$ is not included in $\mathrm{H}_{0}^{1}(\Omega)$.

Remark 4.3. The operator $L$ is injective.

Indeed, let a function $\psi_{h} \in V_{h}$ be such that $\psi_{h}\left(M_{i k}\right)=0 \quad \forall(i, k)$. Then, of course, $\left(\nabla \psi_{h}\right)_{\mid D_{j}}=0$, which implies, as seen in Section 3.2, that $\psi$ vanishes in $V$.

Thanks to Proposition 4.1, we are able to state:

Proposition 4.4. The proposed finite volume method is equivalent to the following non-conforming finite element method:

Find $\phi_{h} \in V_{h}$ such that $\forall \psi_{h} \in V_{h}$,

$$
a_{h}\left(\phi_{h}, \psi_{h}\right)=\ell\left(\psi_{h}\right)
$$

with

$$
a_{h}\left(\phi_{h}, \psi_{h}\right):=\sum_{j \in[1, J]} \int_{D_{j}} \nabla \phi_{h} \cdot \nabla \psi_{h}(\mathbf{x}) \mathrm{d} \mathbf{x} \text { and } \ell\left(\psi_{h}\right):=\int_{\Omega} f \psi_{h}^{*}(\mathbf{x}) \mathrm{d} \mathbf{x}
$$

where $\psi_{h}^{*}$ is defined by (16).

Proof. Let us consider $\psi_{h} \in V_{h}$. Then, there exists one and only one $\psi \in V$ such that $\psi_{h}=L(\psi)$. Taking into account (11) and (12), we may write

$$
\begin{aligned}
-\left|T_{i}\right|\left(\nabla_{h}^{T} \cdot\left(\nabla_{h} \phi\right)\right)_{i} \psi_{i}^{T} & =\left|T_{i}\right| f_{i}^{T} \psi_{i}^{T} \quad \forall i \in[1, I] \\
-\left|P_{k}\right|\left(\nabla_{h}^{P} \cdot\left(\nabla_{h} \phi\right)\right)_{k} \psi_{k}^{P} & =\left|P_{k}\right| f_{k}^{P} \psi_{k}^{P} \quad \forall k \in\left[1, K-J^{\Gamma}\right] .
\end{aligned}
$$

Moreover, as $\psi_{k}^{P}=0 \forall k \in\left[K-J^{\Gamma}+1, K\right]$, there holds

$$
-\left|P_{k}\right|\left(\nabla_{h}^{P} \cdot\left(\nabla_{h} \phi\right)\right)_{k} \psi_{k}^{P}=\left|P_{k}\right| f_{k}^{P} \psi_{k}^{P} \quad \forall k \in[1, K]
$$


which implies that

$$
-\left(\nabla_{h} \cdot\left(\nabla_{h} \phi\right), \psi\right)_{T, P}=(f, \psi)_{T, P} .
$$

Thanks to (8) and to the boundary conditions of the elements of $V$, the left-hand side of (36) is equal to:

$$
\left(\nabla_{h} \phi, \nabla_{h} \psi\right)_{D}
$$

which can also be written, thanks to (32):

$$
\sum_{j \in[1, J]} \int_{D_{j}} \nabla \phi_{h} \cdot \nabla \psi_{h}(\mathbf{x}) \mathrm{d} \mathbf{x}
$$

By Equation (13), and because $\psi_{i}^{T} \theta_{i}^{T}(\mathbf{x})_{\mid T_{i}}=\psi_{i}^{T}$ and $\psi_{k}^{P} \theta_{k}^{P}(\mathbf{x})_{\mid P_{k}}=\psi_{k}^{P}$, the right-hand side of (36) is equal to:

$$
\int_{\Omega} f(\mathbf{x}) \frac{1}{2}\left(\sum_{i \in[1, I]} \psi_{i}^{T} \theta_{i}^{T}(\mathbf{x})+\sum_{k \in[1, K]} \psi_{k}^{P} \theta_{k}^{P}(\mathbf{x})\right) \mathrm{d} \mathbf{x},
$$

which ends this part of the proof.

Conversely, let $\phi_{h} \in V_{h}$ check (34) for all $\psi_{h} \in V_{h}$; then $\phi=L^{-1}\left(\phi_{h}\right)$ checks the boundary conditions (14) by definition. Let us consider $i_{0} \in[1, I]$ and let $\psi$ be defined by

$$
\forall i \in\left[1, I+J^{\Gamma}\right] \psi_{i}^{T}=\delta_{i}^{i_{0}} \text { and } \forall k \in[1, K] \quad \psi_{k}^{P}=0 .
$$

We have

$$
\sum_{j \in[1, J]} \int_{D_{j}} \nabla \phi_{h} \cdot \nabla \psi_{h}(\mathbf{x}) \mathrm{d} \mathbf{x}=\left(\nabla_{h} \phi, \nabla_{h} \psi\right)_{D}=-\left(\nabla_{h} \cdot\left(\nabla_{h} \phi\right), \psi\right)_{T, P}=-\frac{1}{2}\left|T_{i_{0}}\right|\left(\nabla_{h}^{T} \cdot\left(\nabla_{h} \phi\right)\right)_{i_{0}}
$$

and

$$
\int_{\Omega} f \psi_{h}^{*}(\mathbf{x}) \mathrm{d} \mathbf{x}=\frac{1}{2}\left(\sum_{i \in[1, I]}\left|T_{i}\right| f_{i}^{T} \psi_{i}^{T}+\sum_{k \in[1, K]}\left|P_{k}\right| f_{k}^{P} \psi_{k}^{P}\right)=\frac{1}{2}\left|T_{i_{0}}\right| f_{i_{0}}^{T} .
$$

Thus, for this $i_{0}$, the equality

$$
-\left(\nabla_{h}^{T} \cdot\left(\nabla_{h} \phi\right)\right)_{i_{0}}=f_{i_{0}}^{T}
$$

holds. For $k_{0} \in\left[1, K-J^{\Gamma}\right]$, we obtain in the same way

$$
-\left(\nabla_{h}^{P} \cdot\left(\nabla_{h} \phi\right)\right)_{k_{0}}=f_{k_{0}}^{P}
$$

by using $\psi \in V$ defined by

$$
\forall i \in\left[1, I+J^{\Gamma}\right] \psi_{i}^{T}=0 \text { and } \forall k \in[1, K] \psi_{k}^{P}=\delta_{k}^{k_{0}},
$$

which concludes the proof.

We note that this finite element method is a generalization to non-Voronoi meshes of that considered in [25]. 


\section{ERror estimates in the $\mathrm{H}_{0}^{1}$ NORM}

In the literature, two main approaches are used to obtain error estimates for finite volume methods for elliptic equations. In the first, one starts directly from the finite volume formulation and obtains an error estimation in a discrete norm thanks to the consistency of the fluxes along the edges of the mesh; we refer for example to $[6,9,20]$. In the second, the finite volume method is shown to be related to some finite element method for which standard interpolation and consistency errors yield the error estimate for the finite volume method; we refer for example to $[3,25]$.

In the present study, we shall follow both approaches and exhibit some links between them. In the finite volume approach, the fact that the consistency of the discrete gradient operator implies convergence is given by Equation (58) below. The consistency error is then estimated by splitting it into two terms, one of which is the interpolation error of the classical $P^{1}$ interpolant on sub-triangles of the cells $D_{j}$ (see Lem. 5.11). Moreover, the error in the finite volume setting may easily be bounded by the sum of the error in the finite element sense and of the interpolation error between the exact solution and the space $V_{h}$ (see Sect. 5.4).

The advantage of the finite element approach is that it yields a convergence result with a weaker hypothesis. On the other hand, we found it more convenient to treat the case of non-convex diamond cells with the finite volume approach.

We start with a result that will be used throughout the sequel of this article.

Proposition 5.1. The diameters of the diamond-cells are bounded by $2 h$.

$$
\forall j \in[1, J], \operatorname{diam}\left(D_{j}\right) \leq 2 h .
$$

Proof. Consider the partition $D_{j}=D_{j, 1} \cup D_{j, 2}$ (see Fig. 6). As $G_{i} \in T_{i} \forall i \in[1, I]$, we have $D_{j, \alpha} \subset T_{i_{\alpha}(j)}$ for $\alpha \in\{1 ; 2\}$.

To obtain error estimates, it is traditional to suppose that the exact solution $\hat{\phi}$ of the continuous problem is regular. The computational domain being polygonal and convex, a sufficient condition is that the data $f$ is itself regular. We thus make the following assumption

Hypothesis 5.2. The right-hand side $f$ belongs to $\mathrm{L}^{2}(\Omega)$.

Then, we know that the solution $\hat{\phi}$ is in $\mathrm{H}_{0}^{1}(\Omega) \cap \mathrm{H}^{2}(\Omega)$, and that there exists a constant $K$ such that

$$
\|\hat{\phi}\|_{2, \Omega} \leq K\|f\|_{0, \Omega}
$$

We shall see in the following paragraphs that this assumption is sufficient to obtain an optimal error estimate in the $\mathrm{H}_{0}^{1}$ norm. But by supposing more regularity on $f$, and thus also on the solution $\hat{\phi}$, we shall be able to specify the dependence of this error with respect to the geometry of the diamond-cells, and in addition to obtain refined estimates in certain particular cases. We thus state the following assumption:

Hypothesis 5.3. The right-hand side $f$ belongs to $\mathrm{H}^{1}(\Omega)$.

Then, we know that the solution $\hat{\phi}$ is in $\mathrm{H}_{0}^{1}(\Omega) \cap \mathrm{H}^{3}(\Omega)$, and that there exists a constant $K$ such that

$$
\|\hat{\phi}\|_{3, \Omega} \leq K\|f\|_{1, \Omega} .
$$

Since the functions of $\mathrm{H}^{2}(\Omega)$ are continuous, we can consider the values of $\hat{\phi}$ at the points $G_{i}$ and $S_{k}$ and compare them with the values $\phi_{i}^{T}$ and $\phi_{k}^{P}$ resulting from the numerical scheme.

Definition 5.4. We thus define, for any continuous function $v$, the following element $\Pi v$, by

$$
\begin{array}{rlr}
\forall i \in\left[1, I+J^{\Gamma}\right], & (\Pi v)_{i}^{T}=v\left(G_{i}\right) \\
\forall k \in[1, K], & (\Pi v)_{k}^{P}=v\left(S_{k}\right) .
\end{array}
$$




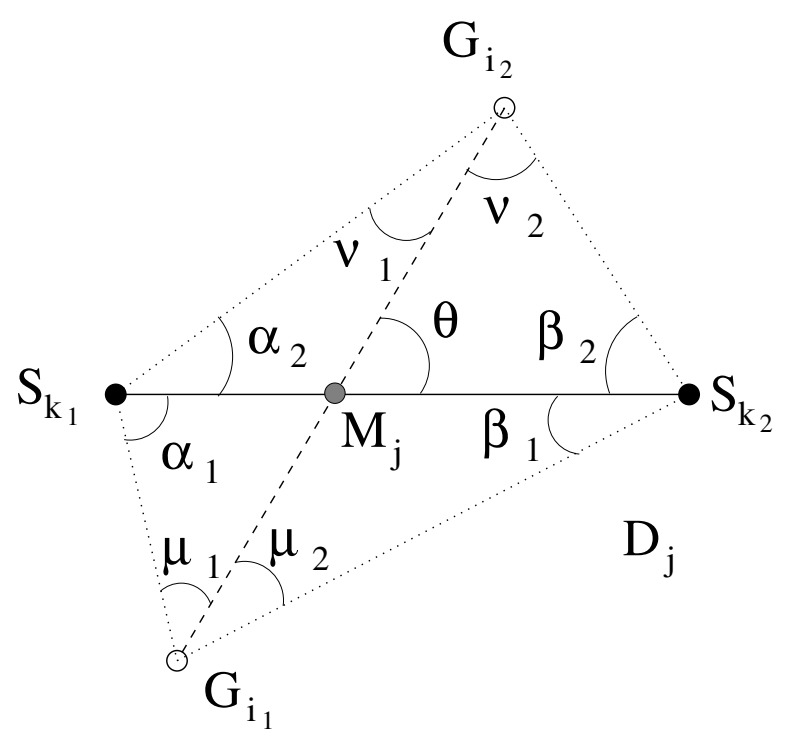

FiguRE 7. Notations for Section 5.1

Note that since $\hat{\phi} \in \mathrm{H}_{0}^{1}(\Omega)$, then $(\Pi \hat{\phi})_{i}^{T}=0$ for $i \in\left[I+1, I+J^{\Gamma}\right]$ and $(\Pi \hat{\phi})_{k}^{P}=0$ for $k \in\left[K-J^{\Gamma}+1, K\right]$, and thus $\Pi \hat{\phi}$ belongs to the space $V$ defined in (15).

We shall suppose in parts 5.2 and 5.3 that all diamond-cells are convex so that we can split each interior diamond-cell $D_{j}$ into two triangles in two ways (see Fig. 6). On the one hand $D_{j}=D_{j, 1} \cup D_{j, 2}$ and on the other hand $D_{j}=D_{j, 1}^{\prime} \cup D_{j, 2}^{\prime}$. In order to simplify our notations, we shall write $\mathcal{T}_{j, \alpha}$ to represent $D_{j, \alpha}$ or $D_{j, \alpha}^{\prime}$, according to the choice carried out. For diamond-cells located on the boundary, let us note that we have $D_{j, 1}=D_{j}$ and $D_{j, 2}=\emptyset$. The case of non-convex diamond-cells is treated in Sect. 5.5.

\subsection{Geometrical hypotheses on the diamond-cells}

When obtaining error estimates, we will use the following assumptions concerning the diamond-cells.

Hypothesis 5.5. The angles between the diagonals of the diamond-cells are greater than an angle $\theta^{*}$ which is strictly positive and independent of the mesh (see Fig. 7 for the notations):

$$
\exists \theta^{*}, 0<\theta^{*} \leq \frac{\pi}{2} \text { s.t. } \theta \geq \theta^{*} .
$$

Hypothesis 5.6. For any diamond-cell $D_{j}$, the smallest in the maximum angle of the couple of triangles $\left(D_{j, 1}, D_{j, 2}\right)$ or in the maximum angle of the couple of triangles $\left(D_{j, 1}^{\prime}, D_{j, 2}^{\prime}\right)$ is bounded by an angle $\tau^{*}$ which is strictly lower than $\pi$ and independent of the mesh:

$$
\exists \tau^{*}<\pi \text {, s.t. } \tau:=\min \left(\max \left(\alpha_{1}, \beta_{1}, \mu_{1}+\mu_{2}, \alpha_{2}, \beta_{2}, \nu_{1}+\nu_{2}\right), \max \left(\mu_{1}, \nu_{1}, \alpha_{1}+\alpha_{2}, \mu_{2}, \nu_{2}, \beta_{1}+\beta_{2}\right)\right) \leq \tau^{*} \text {. }
$$

Proposition 5.7. Hypotheses 5.5 and 5.6 are not equivalent, and, for convex diamond-cells, the second is a consequence of the first.

Proof. Let us consider the diamond-cell represented in Figure 7; let us suppose that it checks assumption (41). Considering the triangle $S_{k_{1}} G_{i_{1}} M_{j}$, we have

$$
\alpha_{1} \leq \pi-\theta \leq \pi-\theta^{*} \leq \pi-\frac{\theta^{*}}{2} .
$$


In the same way,

$$
\mu_{1} \leq \pi-\frac{\theta^{*}}{2}
$$

By considering the triangle $G_{i_{1}} S_{k_{2}} M_{j}$, we can write

$$
\beta_{1} \leq \theta \leq \frac{\pi}{2} \leq \pi-\frac{\theta^{*}}{2}
$$

In the same way,

We have in the same way,

$$
\mu_{2} \leq \pi-\frac{\theta^{*}}{2}
$$

$$
\beta_{2} \leq \pi-\theta \leq \pi-\theta^{*} \leq \pi-\frac{\theta^{*}}{2}
$$

and

$$
\nu_{2} \leq \pi-\frac{\theta^{*}}{2}
$$

And finally,

$$
\alpha_{2} \leq \theta \leq \frac{\pi}{2} \leq \pi-\frac{\theta^{*}}{2}
$$

and

$$
\nu_{1} \leq \pi-\frac{\theta^{*}}{2}
$$

Then, we have the following alternative. Either the following two inequalities are true

$$
\begin{aligned}
& \nu_{1}+\nu_{2} \leq \pi-\frac{\theta^{*}}{2} \text { and } \\
& \mu_{1}+\mu_{2} \leq \pi-\frac{\theta^{*}}{2}
\end{aligned}
$$

and the maximum angle of the couple of triangles $\left(D_{j, 1}, D_{j, 2}\right)$, which is equal to $\max \left(\alpha_{1}, \beta_{1}, \mu_{1}+\mu_{2}, \alpha_{2}, \beta_{2}\right.$, $\left.\nu_{1}+\nu_{2}\right)$, is bounded by $\pi-\frac{\theta^{*}}{2}$, thanks to $(43),(45),(52),(49),(47)$ and $(51)$, or at least one of the two inequalities (51) or (52) is false. In this case, let us suppose for example that $\mu_{1}+\mu_{2} \geq \pi-\frac{\theta^{*}}{2}$. Then, we have

$$
\alpha_{1}+\beta_{1}=\pi-\mu_{1}-\mu_{2} \leq \frac{\theta^{*}}{2} .
$$

Because $\alpha_{1}$ and $\beta_{1}$ are both positive, there holds

$$
\alpha_{1} \leq \frac{\theta^{*}}{2} \text { and } \beta_{1} \leq \frac{\theta^{*}}{2} .
$$

So that, taking into account the second inequality in (49),

$$
\alpha_{1}+\alpha_{2} \leq \frac{\pi}{2}+\frac{\theta^{*}}{2} \leq \pi-\frac{\theta^{*}}{2}\left(\text { because } \theta^{*} \leq \frac{\pi}{2}\right)
$$

and, taking into account (47),

$$
\beta_{1}+\beta_{2} \leq \pi-\frac{\theta^{*}}{2}
$$

Thus, thanks to $(44),(50),(53),(46),(48)$ and $(54)$, the maximum angle of the couple of triangles $\left(D_{j, 1}^{\prime}, D_{j, 2}^{\prime}\right)$, which is equal to $\max \left(\mu_{1}, \nu_{1}, \alpha_{1}+\alpha_{2}, \mu_{2}, \nu_{2}, \beta_{1}+\beta_{2}\right)$, is bounded by $\pi-\frac{\theta^{*}}{2}$. Thus, we have proved that the condition (42) is indeed a consequence of (41) by choosing $\tau^{*}=\pi-\frac{\theta^{*}}{2}$. 
On the other hand, to show that (42) may be checked even if (41) is not, let us consider a rectangular diamond-cell, with length $a$ and width $b$. If $a$ is fixed while we let $b$ tend to 0 , then the angle $\theta$ between the diagonals of this rectangle will tend to 0 , while the angle $\tau$ defined by (42) obviously remains equal to $\frac{\pi}{2}$.

Remark 5.8. Of course, Hypotheses 5.5 and 5.6 have to be considered when one deals with families of increasingly fine meshes. Indeed, for a single mesh, (41) is checked for $\theta^{*}=\min _{j \in[1, J]} \theta_{j}$, where $\theta_{j}$ is the angle between the diagonals of the diamond-cell $D_{j}$. A way to construct families of meshes that a priori verify these hypotheses is beyond the scope of this article; on the other hand, it is an easy matter to compute a posteriori the angles $\theta^{*}$ and $\tau^{*}$ for a given mesh. We note that the family of non-conforming meshes considered in Section 8 satisfies Hypothesis 5.5 with an angle $\theta^{*}=\frac{\pi}{4}$.

\subsection{Finite volume approach}

Like in [9], we estimate the discrete norm of the error between $\phi$, the element of $V$ solution of the system (11)-(14) and the projection of the exact solution $\Pi \hat{\phi}$ (see Def. 5.4). Let us note $\varepsilon:=\phi-\Pi \hat{\phi}$.

Let us define on each diamond-cell $D_{j}$ the quantity $\mathbf{e}_{j}(\mathbf{x}):=(\delta \hat{\phi})_{j}-\nabla \hat{\phi}(\mathbf{x})$. Further, let us split $D_{j}$ into $\mathcal{T}_{j, 1} \cup \mathcal{T}_{j, 2}$ (let us recall that these notations are defined just before the beginning of Section 5.1; the way this splitting is performed is local to $D_{j}$ ). Let $\omega_{j, 1}$ (respectively $\omega_{j, 2}$ ) be the traditional $P^{1}$ interpolant of $\hat{\phi}$ on the triangle $\mathcal{T}_{j, 1}\left(\operatorname{resp} . \mathcal{T}_{j, 2}\right)$ whose value at each of the three nodes of $\mathcal{T}_{j, 1}$ (resp. $\mathcal{T}_{j, 2}$ ) is equal to the value of the function $\hat{\phi}$ at this point.

Lemma 5.9. For convex diamond-cells, we have the following inequality (recalling that $|\varepsilon|_{1, D}=\left(\nabla_{h} \varepsilon, \nabla_{h} \varepsilon\right)_{D}^{1 / 2}$ )

$$
|\varepsilon|_{1, D} \leq\left(\sum_{j \in[1, J]} \int_{D_{j}}\left|\mathbf{e}_{j}\right|^{2}(\mathbf{x}) \mathrm{d} \mathbf{x}\right)^{1 / 2}+\left(\sum_{j \in[1, J]} \sum_{\alpha=1}^{2} \int_{\mathcal{T}_{j, \alpha}}\left|\nabla \hat{\phi}(\mathbf{x})-\nabla \omega_{j, \alpha}\right|^{2} \mathrm{~d} \mathbf{x}\right)^{1 / 2} .
$$

Proof. By Equation (11), we have

$$
\left(\nabla_{h}^{T} \cdot\left(\nabla_{h} \phi\right)\right)_{i}=-\frac{1}{\left|T_{i}\right|} \int_{T_{i}} f \mathrm{~d} \mathbf{x}=\frac{1}{\left|T_{i}\right|} \int_{T_{i}} \Delta \hat{\phi} \mathrm{d} \mathbf{x} .
$$

Then, we can write for all $i \in[1, I]$

$$
\left(\nabla_{h}^{T} \cdot\left(\nabla_{h} \varepsilon\right)\right)_{i}=\frac{1}{\left|T_{i}\right|} \int_{T_{i}} \Delta \hat{\phi} \mathrm{d} \mathbf{x}-\left(\nabla_{h}^{T} \cdot\left(\nabla_{h} \Pi \hat{\phi}\right)\right)_{i} .
$$

And thus

$$
\left(\nabla_{h}^{T} \cdot\left(\nabla_{h} \varepsilon\right)\right)_{i} \varepsilon_{i}^{T}=\left[\frac{1}{\left|T_{i}\right|} \int_{T_{i}} \Delta \hat{\phi} \mathrm{d} \mathbf{x}-\left(\nabla_{h}^{T} \cdot\left(\nabla_{h} \Pi \hat{\phi}\right)\right)_{i}\right] \varepsilon_{i}^{T} .
$$

In the same way, for all $k \in\left[1, K-J^{\Gamma}\right]$,

$$
\left(\nabla_{h}^{P} \cdot\left(\nabla_{h} \varepsilon\right)\right)_{k} \varepsilon_{k}^{P}=\left[\frac{1}{\left|P_{k}\right|} \int_{P_{k}} \Delta \hat{\phi} \mathrm{d} \mathbf{x}-\left(\nabla_{h}^{P} \cdot\left(\nabla_{h} \Pi \hat{\phi}\right)\right)_{k}\right] \varepsilon_{k}^{P} .
$$

This equality is also true for all $k \in\left[K-J^{\Gamma}+1, K\right]$ since then $\varepsilon_{k}^{P}=0$. Summing (56) and (57), we can write

$$
\begin{aligned}
|\varepsilon|_{1, D}^{2} & =-\left(\nabla_{h} \cdot\left(\nabla_{h} \varepsilon\right)_{,} \varepsilon\right)_{T, P} \\
& =-\left(\Delta \hat{\phi}, \varepsilon_{h}^{*}\right)_{\Omega}+\left(\nabla_{h} \cdot\left(\nabla_{h} \Pi \hat{\phi}\right), \varepsilon\right)_{T, P}=-\left(\Delta \hat{\phi}, \varepsilon_{h}^{*}\right)_{\Omega}-\left(\nabla_{h} \Pi \hat{\phi}, \nabla_{h} \varepsilon\right)_{D} \\
& =\left(\delta \hat{\phi}-\nabla_{h} \Pi \hat{\phi}, \nabla_{h} \varepsilon\right)_{D},
\end{aligned}
$$


the last equality in (58) being obtained thanks to (20). Thus,

$$
\begin{aligned}
|\varepsilon|_{1, D}^{2} & =\left(\delta \hat{\phi}-\nabla_{h} \Pi \hat{\phi}, \nabla_{h} \varepsilon\right)_{D}=\sum_{j \in[1, J]}\left|D_{j}\right|\left(\delta \hat{\phi}-\nabla_{h} \Pi \hat{\phi}\right)_{j} \cdot\left(\nabla_{h} \varepsilon\right)_{j}=\sum_{j \in[1, J]} \int_{D_{j}}\left(\delta \hat{\phi}-\nabla_{h} \Pi \hat{\phi}\right)_{j} \cdot\left(\nabla_{h} \varepsilon\right)_{j} \mathrm{~d} \mathbf{x} \\
& =\sum_{j \in[1, J]} \int_{D_{j}} \mathbf{e}_{j}(\mathbf{x}) \cdot\left(\nabla_{h} \varepsilon\right)_{j} \mathrm{~d} \mathbf{x}+\sum_{j \in[1, J]} \int_{D_{j}}\left(\nabla \hat{\phi}(\mathbf{x})-\left(\nabla_{h} \Pi \hat{\phi}\right)_{j}\right) \cdot\left(\nabla_{h} \varepsilon\right)_{j} \mathrm{~d} \mathbf{x}
\end{aligned}
$$

Let us first bound the first term in (59). By the Cauchy-Schwarz inequality, and then the discrete CauchySchwarz inequality, we may write

$$
\begin{aligned}
\sum_{j \in[1, J]} \int_{D_{j}} \mathbf{e}_{j}(\mathbf{x}) \cdot\left(\nabla_{h} \varepsilon\right)_{j} \mathrm{~d} \mathbf{x} & \leq \sum_{j \in[1, J]}\left(\int_{D_{j}}\left|\mathbf{e}_{j}\right|^{2}(\mathbf{x}) \mathrm{d} \mathbf{x}\right)^{1 / 2}\left(\int_{D_{j}}\left|\left(\nabla_{h} \varepsilon\right)_{j}\right|^{2} \mathrm{~d} \mathbf{x}\right)^{1 / 2} \\
& \leq\left(\sum_{j \in[1, J]} \int_{D_{j}}\left|\mathbf{e}_{j}\right|^{2}(\mathbf{x}) \mathrm{d} \mathbf{x}\right)^{1 / 2}\left(\sum_{j \in[1, J]} \int_{D_{j}}\left|\left(\nabla_{h} \varepsilon\right)_{j}\right|^{2} \mathrm{~d} \mathbf{x}\right)^{1 / 2} \\
& \leq\left(\sum_{j \in[1, J]} \int_{D_{j}}\left|\mathbf{e}_{j}\right|^{2}(\mathbf{x}) \mathrm{d} \mathbf{x}\right)^{1 / 2}|\varepsilon|_{1, D} .
\end{aligned}
$$

Moreover, an elementary calculation proves that, on each convex diamond-cell $D_{j}$,

$$
\int_{D_{j}}\left(\nabla_{h} \Pi \hat{\phi}\right)_{j} \mathrm{~d} \mathbf{x}=\int_{\mathcal{T}_{j, 1}} \nabla \omega_{j, 1}(\mathbf{x}) \mathrm{d} \mathbf{x}+\int_{\mathcal{T}_{j, 2}} \nabla \omega_{j, 2}(\mathbf{x}) \mathrm{d} \mathbf{x}
$$

Thus, $\left(\nabla_{h} \varepsilon\right)_{j}$ being a constant on each cell $D_{j}$,

$$
\int_{D_{j}}\left(\nabla \hat{\phi}(\mathbf{x})-\left(\nabla_{h} \Pi \hat{\phi}\right)_{j}\right) \cdot\left(\nabla_{h} \varepsilon\right)_{j} \mathrm{~d} \mathbf{x}=\sum_{\alpha=1}^{2} \int_{\mathcal{T}_{j, \alpha}}\left(\nabla \hat{\phi}(\mathbf{x})-\nabla \omega_{j, \alpha}\right) \cdot\left(\nabla_{h} \varepsilon\right)_{j}(\mathbf{x}) \mathrm{d} \mathbf{x}
$$

Applying as above Cauchy-Schwarz inequalities, we can write

$$
\sum_{j \in[1, J]} \int_{D_{j}}\left(\nabla \hat{\phi}(\mathbf{x})-\left(\nabla_{h} \Pi \hat{\phi}\right)_{j}\right) \cdot\left(\nabla_{h} \varepsilon\right)_{j} \mathrm{~d} \mathbf{x} \leq\left(\sum_{j \in[1, J]} \sum_{\alpha=1}^{2} \int_{\mathcal{T}_{j, \alpha}}\left|\nabla \hat{\phi}(\mathbf{x})-\nabla \omega_{j, \alpha}\right|^{2} \mathrm{~d} \mathbf{x}\right)^{1 / 2}|\varepsilon|_{1, D} .
$$

The result follows from (59), (60) and (62).

An estimation of the first term in Equation (55) is given by the following lemma

Lemma 5.10. If all diamond-cells are convex and under Hypothesis 5.5, there exists a constant $C$ independent of the grid, such that the following inequality holds

$$
\left(\sum_{j \in[1, J]} \int_{D_{j}}\left|\mathbf{e}_{j}\right|^{2}(\mathbf{x}) \mathrm{d} \mathbf{x}\right)^{1 / 2} \leq C \frac{h}{\sin \theta^{*}}\|f\|_{0, \Omega}
$$


Proof. We can bound the term $\left|\mathbf{e}_{j}\right|^{2}$ (x) using the respective scalar products of $\mathbf{e}_{j}$ with $\mathbf{n}_{j}$ and $\mathbf{n}_{j}^{\prime}$. An elementary calculation proves that

And we have

$$
\left|\mathbf{e}_{j}\right|^{2} \leq \frac{2}{1-\left(\mathbf{n}_{j} \cdot \mathbf{n}_{j}^{\prime}\right)^{2}}\left[\left(\mathbf{e}_{j} \cdot \mathbf{n}_{j}\right)^{2}+\left(\mathbf{e}_{j} \cdot \mathbf{n}_{j}^{\prime}\right)^{2}\right]
$$

$$
\int_{D_{j}}\left(\mathbf{e}_{j} \cdot \mathbf{n}_{j}\right)^{2}(\mathbf{x}) \mathrm{d} \mathbf{x}=\sum_{\alpha=1}^{2}\left\|\mathbf{e}_{j} \cdot \mathbf{n}_{j}\right\|_{0, D_{j, \alpha}}^{2} .
$$

Then, we remark that the function $\mathbf{e}_{j} \cdot \mathbf{n}_{j}(\mathbf{x})=\left((\delta \hat{\phi})_{j}-\nabla \hat{\phi}(\mathbf{x})\right) \cdot \mathbf{n}_{j}$ is in $\mathrm{H}^{1}\left(D_{j, \alpha}\right)$ and that, by construction of $(\delta \hat{\phi})_{j}$, its average vanishes on $A_{j}$, which is an edge of $D_{j, \alpha}$. Thanks to [1], lemma 2.2, we know that there exists a constant $C$, independent of $j, \alpha$ and $h$, such that

$$
\left\|\mathbf{e}_{j} \cdot \mathbf{n}_{j}\right\|_{0, D_{j, \alpha}} \leq \operatorname{Cdiam}\left(D_{j, \alpha}\right)\left|\mathbf{e}_{j} \cdot \mathbf{n}_{j}\right|_{1, D_{j, \alpha}} \leq C h|\hat{\phi}|_{2, D_{j, \alpha}}
$$

since the gradient of $(\delta \hat{\phi})_{j} \cdot \mathbf{n}_{j}$ vanishes. Thus,

$$
\int_{D_{j}}\left(\mathbf{e}_{j} \cdot \mathbf{n}_{j}\right)^{2}(\mathbf{x}) \mathrm{d} \mathbf{x} \leq C h^{2}|\hat{\phi}|_{2, D_{j}}^{2} .
$$

In the same way, since the cell $D_{j}$ is convex, we can carry out the same operations for the other component of $\mathbf{e}_{j}$ and we obtain

$$
\int_{D_{j}}\left(\mathbf{e}_{j} \cdot \mathbf{n}_{j}^{\prime}\right)^{2}(\mathbf{x}) \mathrm{d} \mathbf{x} \leq C h^{2}|\hat{\phi}|_{2, D_{j}}^{2} .
$$

Finally, since $1-\left(\mathbf{n}_{j} \cdot \mathbf{n}_{j}^{\prime}\right)^{2}$ is the square of the sine of the angle between the diagonals of the diamond-cell $D_{j}$, and under assumption 5.5, we can prove the existence of a constant $C$, independent of the grid, such that

$$
\int_{D_{j}}\left|\mathbf{e}_{j}\right|^{2}(\mathbf{x}) \mathrm{d} \mathbf{x} \leq C \frac{h^{2}}{\left(\sin \theta^{*}\right)^{2}}|\hat{\phi}|_{2, D_{j}}^{2} .
$$

Summing this inequality over the diamond-cells, and thanks to (37), we obtain the result.

An estimation of the second term in Equation (55) is given by the following lemma:

Lemma 5.11. If all diamond-cells are convex and under assumptions 5.2 and 5.6, there exists a constant $C$ independent of the grid such that

$$
\left(\sum_{j \in[1, J]} \sum_{\alpha=1}^{2} \int_{\mathcal{T}_{j, \alpha}}\left|\nabla \hat{\phi}(\mathbf{x})-\nabla \omega_{j, \alpha}\right|^{2} \mathrm{~d} \mathbf{x}\right)^{1 / 2} \leq C\left(\tau^{*}\right) h\|f\|_{0, \Omega} .
$$

Moreover, under assumptions 5.3 and 5.6, there exists a constant $C$ independent of the grid such that

$$
\left(\sum_{j \in[1, J]} \sum_{\alpha=1}^{2} \int_{\mathcal{T}_{j, \alpha}}\left|\nabla \hat{\phi}(\mathbf{x})-\nabla \omega_{j, \alpha}\right|^{2} \mathrm{~d} \mathbf{x}\right)^{1 / 2} \leq C \frac{h}{\cos \frac{\tau^{*}}{2}}\left(\|f\|_{0, \Omega}+h\|f\|_{1, \Omega}\right) .
$$

Proof. When $D_{j}$ is convex and under Assumption 5.6, it is possible to choose the splitting of $D_{j}$ so that all triangles $\mathcal{T}_{j, \alpha}$ check the assumption known as the maximum angle condition: the maximum angle of these triangles is bounded by an angle $\tau^{*}$ which is strictly lower than $\pi$. Under Hypothesis 5.2 , the solution $\hat{\phi}$ is 

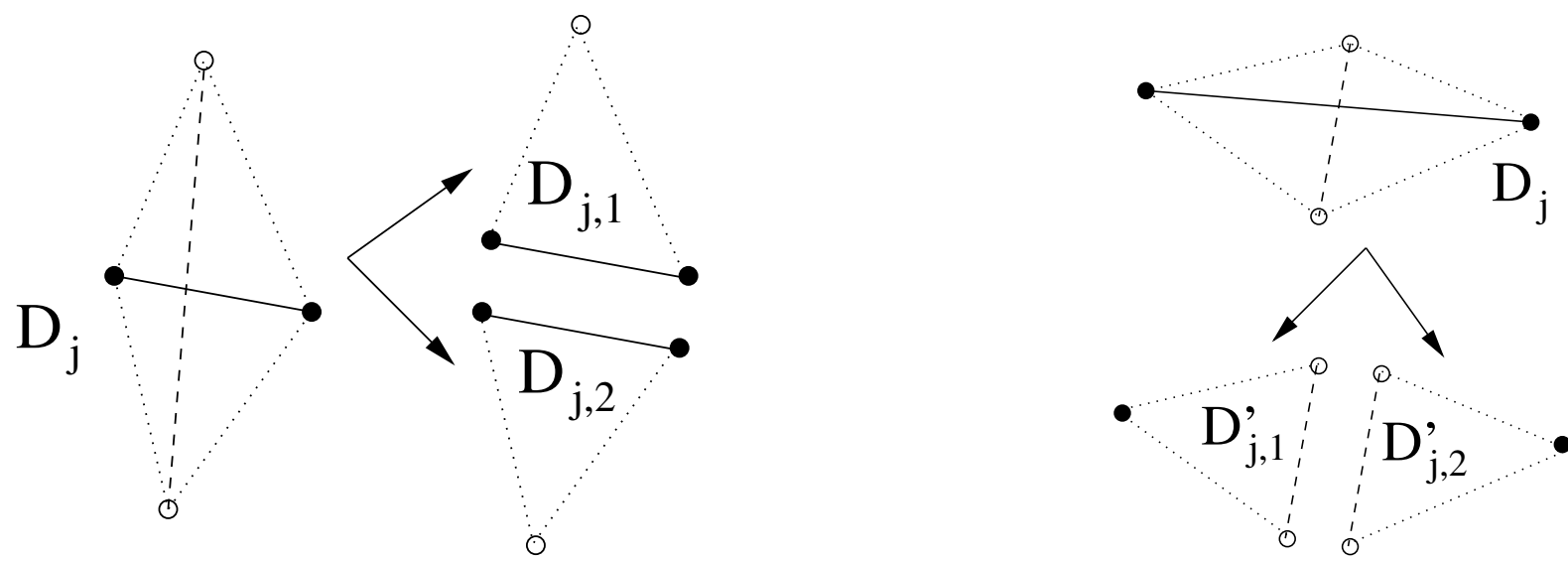

Figure 8. Two diamond-cells for which the error estimate is favorable.

in $\mathrm{H}^{2}(\Omega)$ and we use the error estimate obtained in [2], Theorem 2.3, to state that there exists a constant $C$ independent of $j, \alpha$ and $h$ such that

$$
\left|\hat{\phi}-\omega_{j, \alpha}\right|_{1, \mathcal{T}_{j, \alpha}} \leq C\left(\tau^{*}\right) \operatorname{diam}\left(\mathcal{T}_{j, \alpha}\right)|\hat{\phi}|_{2, \mathcal{T}_{j, \alpha}} \leq C\left(\tau^{*}\right) h|\hat{\phi}|_{2, \mathcal{T}_{j, \alpha}}
$$

If Hypothesis 5.3 is checked, then the solution $\hat{\phi}$ is in $\mathrm{H}^{3}(\Omega)$ and we can specify the dependence of the constant $C\left(\tau^{*}\right)$, thanks to [18], Theorem 3.1-ii:

$$
\left|\hat{\phi}-\omega_{j, \alpha}\right|_{1, \mathcal{T}_{j, \alpha}} \leq C \frac{h}{\cos \frac{\tau^{*}}{2}}\left(|\hat{\phi}|_{2, \mathcal{T}_{j, \alpha}}+h|\hat{\phi}|_{3, \mathcal{T}_{j, \alpha}}\right)
$$

where the constant $C$ does not depend any more on $\tau^{*}$.

The results follow by summing (68) or (69) over the diamond-cells, and by (37) and (38).

Remark 5.12. It seems very important to insist on the fact that since the cell $D_{j}$ is convex, we can write

$$
\left|\int_{D_{j}}\left(\nabla \hat{\phi}-\left(\nabla_{h} \Pi \hat{\phi}\right)_{j}\right) \cdot\left(\nabla_{h} \varepsilon\right)_{j}\right|^{2} \leq \inf \left(\sum_{\alpha=1}^{2}\left|\hat{\phi}-\omega_{j, \alpha}\right|_{1, D_{j, \alpha}}^{2}, \sum_{\alpha=1}^{2}\left|\hat{\phi}-\omega_{j, \alpha}\right|_{1, D_{j, \alpha}^{\prime}}^{2}\right)\left|D_{j}\right|\left|\nabla_{h} \varepsilon\right|_{j}^{2},
$$

and that it is thus sufficient that one of the two couples of triangles $\left(D_{j, 1}, D_{j, 2}\right)$ or $\left(D_{j, 1}^{\prime}, D_{j, 2}^{\prime}\right)$ does not have an angle too close to $\pi$ for the error estimate to be good. In particular, diamond-cells having two opposite angles close to $\pi$ but four sides of similar lengths do not cause any problem (see Fig. 8). We represent in addition in Figure 9 two examples of cells whose shape will cause the error estimate to be bad.

To conclude, we can state the following result:

Theorem 5.13. If all diamond-cells are convex and under Hypotheses 5.2 and 5.5, there exists a constant $C\left(\theta^{*}\right)$, independent of $h$, such that

$$
|\varepsilon|_{1, D} \leq C\left(\theta^{*}\right) h\|f\|_{0, \Omega} .
$$

Moreover, under Hypotheses 5.3 and 5.5, there exists a constant $C$, independent of $h$ and $\theta^{*}$, such that

$$
|\varepsilon|_{1, D} \leq C \frac{h}{\sin \theta^{*}}\left(\|f\|_{0, \Omega}+h\|f\|_{1, \Omega}\right)
$$




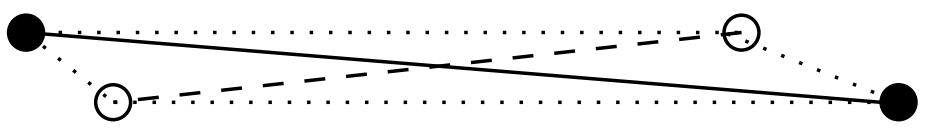

(a) Almost flat quadrangle

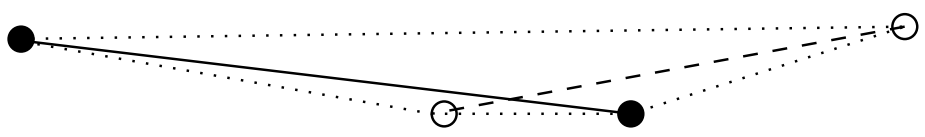

(b) Two consecutive angles close to $\pi$

Figure 9. Examples of diamond-cells for which the error estimate is not favorable.

Proof. This result follows from the discussion held in Section 5.1 and taking into account (55), (63) and (66) for (70) and (55), (63) and (67) for (71).

Remark 5.14. Contrary to [9], Definition 9.4, we do not need to consider a "restricted" class of meshes to obtain uniform error estimates, that is to say the ratio $\frac{\left|A_{j}\right|}{\left|A_{j}^{\prime}\right|}$ need not be bounded.

\subsection{Finite element approach}

Thanks to the equivalence of the finite volume scheme with a finite element scheme, we can attack the error estimation through a more traditional approach, which extends the proof of [25] (given for Voronoi meshes) to arbitrary meshes.

According to formula (34), the proposed scheme amounts to finding $\phi_{h} \in V_{h}$ such that for all $\psi_{h} \in V_{h}$,

$$
a_{h}\left(\phi_{h}, \psi_{h}\right)=\ell\left(\psi_{h}\right)
$$

The error estimate is derived in a traditional way by noting that $a_{h}$ acts on $\mathrm{H}_{0}^{1}+V_{h}$, on which we define $|x|_{1, h}:=\sqrt{a_{h}(x, x)}$, and by using the so-called "Strang second lemma" [24]:

$$
\left|\hat{\phi}-\phi_{h}\right|_{1, h} \leq 2 \inf _{\omega_{h} \in V_{h}}\left|\hat{\phi}-\omega_{h}\right|_{1, h}+\sup _{\omega_{h} \in V_{h}} \frac{\left|a_{h}\left(\hat{\phi}, \omega_{h}\right)-\ell\left(\omega_{h}\right)\right|}{\left|\omega_{h}\right|_{1, h}} .
$$

The first of these two terms is named "interpolation error" and measures the way in which $V_{h}$ approaches $\mathrm{H}_{0}^{1}$, while the second is called "consistency error" and appears only in non-conforming approximations.

\subsubsection{Interpolation error}

Proposition 5.15. If all diamond-cells are convex and under Hypotheses 5.2 and 5.6, there exists a constant $C\left(\tau^{*}\right)$ depending only on $\tau^{*}$ such that

$$
\inf _{\omega_{h} \in V_{h}}\left|\hat{\phi}-\omega_{h}\right|_{1, h} \leq C\left(\tau^{*}\right) h\|f\|_{0, \Omega}
$$

Moreover, under Hypotheses 5.3 and 5.6, we can be more specific: there exists a constant $C$, independent of the grid, such that

$$
\inf _{\omega_{h} \in V_{h}}\left|\hat{\phi}-\omega_{h}\right|_{1, h} \leq C \frac{h}{\cos \frac{\tau^{*}}{2}}\left(\|f\|_{0, \Omega}+h\|f\|_{1, \Omega}\right)
$$

Proof. As a particular element of $V_{h}$, we choose the interpolation $\omega_{h}$ of the exact solution $\hat{\phi}$

$$
\omega_{h}:=L(\Pi \hat{\phi})
$$


where the element $\Pi \hat{\phi}$ of $V$ is defined through (39)-(40). We have, thanks to (32),

$$
\left|\hat{\phi}-\omega_{h}\right|_{1, h}^{2}=\sum_{j \in[1, J]} \int_{D_{j}}\left|\nabla \hat{\phi}-\nabla \omega_{h}\right|^{2}(\mathbf{x}) \mathrm{d} \mathbf{x}=\sum_{j \in[1, J]} \int_{D_{j}}\left|\nabla \hat{\phi}-\left(\nabla_{h} \Pi \hat{\phi}\right)_{j}\right|^{2}(\mathbf{x}) \mathrm{d} \mathbf{x} .
$$

Let us note $\langle\nabla \hat{\phi}\rangle_{j}$ the average value of $\nabla \hat{\phi}$ on $D_{j}$, defined by

$$
\langle\nabla \hat{\phi}\rangle_{j}:=\frac{1}{\left|D_{j}\right|} \int_{D_{j}} \nabla \hat{\phi}(\mathbf{x}) \mathrm{d} \mathbf{x}
$$

We have

$$
\begin{aligned}
\int_{D_{j}}\left|\nabla \hat{\phi}-\left(\nabla_{h} \Pi \hat{\phi}\right)_{j}\right|^{2}(\mathbf{x}) \mathrm{d} \mathbf{x} & =\int_{D_{j}}\left|\nabla \hat{\phi}-\langle\nabla \hat{\phi}\rangle_{j}+\langle\nabla \hat{\phi}\rangle_{j}-\left(\nabla_{h} \Pi \hat{\phi}\right)_{j}\right|^{2}(\mathbf{x}) \mathrm{d} \mathbf{x} \\
& \leq 2 \int_{D_{j}}\left|\nabla \hat{\phi}-\langle\nabla \hat{\phi}\rangle_{j}\right|^{2}(\mathbf{x}) \mathrm{d} \mathbf{x}+2 \int_{D_{j}}\left|\langle\nabla \hat{\phi}\rangle_{j}-\left(\nabla_{h} \Pi \hat{\phi}\right)_{j}\right|^{2}(\mathbf{x}) \mathrm{d} \mathbf{x} .
\end{aligned}
$$

The first of the two terms of the right-hand side of (76) is easily bounded. Indeed, the function $\nabla \hat{\phi}-\langle\nabla \hat{\phi}\rangle_{j}$ has a vanishing average on $D_{j}$, and, $D_{j}$ being convex, Payne and Weinberger [21] indicate that there exists a constant $C$, independent of $j$ and of $h$ such that

$$
\int_{D_{j}}\left|\nabla \hat{\phi}-\langle\nabla \hat{\phi}\rangle_{j}\right|^{2}(\mathbf{x}) \mathrm{d} \mathbf{x} \leq C \operatorname{diam}\left(D_{j}\right)^{2}|\hat{\phi}|_{2, D_{j}}^{2} \leq C h^{2}|\hat{\phi}|_{2, D_{j}}^{2}
$$

The integrand of the second term in the right-hand side of (76) being a constant for each $j$, we have

$$
\begin{aligned}
\int_{D_{j}}\left|\langle\nabla \hat{\phi}\rangle_{j}-\left(\nabla_{h} \Pi \hat{\phi}\right)_{j}\right|^{2}(\mathbf{x}) \mathrm{d} \mathbf{x} & =\frac{1}{\left|D_{j}\right|}\left|\int_{D_{j}}\left(\langle\nabla \hat{\phi}\rangle_{j}-\left(\nabla_{h} \Pi \hat{\phi}\right)_{j}\right)(\mathbf{x}) \mathrm{d} \mathbf{x}\right|^{2} \\
& =\frac{1}{\left|D_{j}\right|}\left|\int_{D_{j}}\left(\nabla \hat{\phi}-\left(\nabla_{h} \Pi \hat{\phi}\right)_{j}\right)(\mathbf{x}) \mathrm{d} \mathbf{x}\right|^{2} \\
& =\frac{1}{\left|D_{j}\right|}\left|\sum_{\alpha=1}^{2} \int_{\mathcal{T}_{j, \alpha}}\left(\nabla \hat{\phi}-\nabla \omega_{j, \alpha}\right)(\mathbf{x}) \mathrm{d} \mathbf{x}\right|^{2}
\end{aligned}
$$

according to (61). By applying the Cauchy-Schwarz inequality, we obtain

$$
\int_{D_{j}}\left|\langle\nabla \hat{\phi}\rangle_{j}-\left(\nabla_{h} \Pi \hat{\phi}\right)_{j}\right|^{2}(\mathbf{x}) \mathrm{d} \mathbf{x} \leq \frac{2}{\left|D_{j}\right|} \sum_{\alpha=1}^{2}\left|\mathcal{T}_{j, \alpha}\right|\left|\hat{\phi}-\omega_{j, \alpha}\right|_{1, \mathcal{T}_{j, \alpha}}^{2} .
$$

As the diamond-cells are convex, we have $\left|\mathcal{T}_{j, \alpha}\right| \leq\left|D_{j}\right|$ and thanks to (68), under Hypotheses 5.2 and 5.6

$$
\int_{D_{j}}\left|\langle\nabla \hat{\phi}\rangle_{j}-\left(\nabla_{h} \Pi \hat{\phi}\right)_{j}\right|^{2}(\mathbf{x}) \mathrm{d} \mathbf{x} \leq C\left(\tau^{*}\right) h^{2}|\hat{\phi}|_{2, D_{j}}^{2} .
$$

Thanks to (76), (77) and (78) we obtain the existence of a constant $C\left(\tau^{*}\right)$ independent of $j$ and $h$ such that

$$
\int_{D_{j}}\left|\nabla \hat{\phi}-\nabla_{h} \Pi \hat{\phi}\right|^{2}(\mathbf{x}) \mathrm{d} \mathbf{x} \leq C\left(\tau^{*}\right) h^{2}|\hat{\phi}|_{2, D_{j}}^{2} .
$$


Moreover, under Hypotheses 5.3 and 5.6, and thanks to (69), there holds

$$
\int_{D_{j}}\left|\langle\nabla \hat{\phi}\rangle_{j}-\left(\nabla_{h} \Pi \hat{\phi}\right)_{j}\right|^{2}(\mathbf{x}) \mathrm{d} \mathbf{x} \leq C \frac{h^{2}}{\left(\cos \frac{\tau^{*}}{2}\right)^{2}}\left(|\hat{\phi}|_{2, D_{j}}+h|\hat{\phi}|_{3, D_{j}}\right)^{2} .
$$

Thanks to (76), (77) and (80), we obtain the existence of a constant $C$ independent of the grid such that

$$
\int_{D_{j}}\left|\nabla \hat{\phi}-\nabla_{h} \Pi \hat{\phi}\right|^{2}(\mathbf{x}) \mathrm{d} \mathbf{x} \leq C \frac{h^{2}}{\left(\cos \frac{\tau^{*}}{2}\right)^{2}}\left(|\hat{\phi}|_{2, D_{j}}+h|\hat{\phi}|_{3, D_{j}}\right)^{2} .
$$

By summation of (79) or (81) over the diamond-cells, and taking into account (37) or (38), we obtain the desired error estimate for the interpolation error.

\subsubsection{Consistency error}

Let $\omega_{h} \in V_{h}$. Thanks to $(35)$, we start by writing

$$
a_{h}\left(\hat{\phi}, \omega_{h}\right)-\ell\left(\omega_{h}\right)=\left[a_{h}\left(\hat{\phi}, \omega_{h}\right)-\left(f, \omega_{h}\right)_{\Omega}\right]+\left(f, \omega_{h}-\omega_{h}^{*}\right)_{\Omega}
$$

The last term in (82) can be bounded by the following lemma:

Lemma 5.16. If all diamond-cells are convex, there exists a constant $C$ independent of the grid such that

$$
\left|\left(f, \omega_{h}-\omega_{h}^{*}\right)_{\Omega}\right| \leq\left. C h|| f\right|_{0, \Omega}\left|\omega_{h}\right|_{1, h} .
$$

Proof. First, on each cell $D_{j}$,

$$
\left|\int_{D_{j}} f\left(\omega_{h}-\omega_{h}^{*}\right)(\mathbf{x}) \mathrm{d} \mathbf{x}\right| \leq\|f\|_{0, D_{j}}\left\|\omega_{h}-\omega_{h}^{*}\right\|_{0, D_{j}}
$$

By definition, the function $\omega_{h}^{*}$ is a constant over each domain $T_{i} \cap P_{k}$, for $i \in[1, I]$ and $k \in[1, K]$, and is equal to $\frac{1}{2}\left(\omega_{i}^{T}+\omega_{k}^{P}\right)$. Since interior diamond-cells are convex, each one of them can be split in the following way (see Fig. 10):

with the definition

$$
D_{j}=\bigcup_{(\alpha, \beta) \in\{1 ; 2\}^{2}} D_{j, \alpha \beta}
$$

$$
D_{j, \alpha \beta}:=D_{j} \cap\left(T_{i_{\alpha}(j)} \cap P_{k_{\beta}(j)}\right) .
$$

We will note $D_{\alpha \beta}$ instead of $D_{j, \alpha \beta}$ when no confusion is possible.

By definition of $\omega_{h}$, we have for each of the four couples $(\alpha, \beta)$,

$$
\omega_{h}\left(M_{i_{\alpha} k_{\beta}}\right)=\frac{1}{2}\left(\omega_{i_{\alpha}}^{T}+\omega_{k_{\beta}}^{P}\right)=\left(\omega_{h}^{*}\right)_{\mid D_{\alpha \beta}} .
$$

In other words, the piecewise constant function $\omega_{h}^{*}$ interpolates $\omega_{h}$ at the points $M_{i k}$. Then, there exists a constant $C$ independent of the grid (see for example [5], Theorem 15.3) such that

$$
\left\|\omega_{h}-\omega_{h}^{*}\right\|_{0, D_{\alpha \beta}} \leq C \operatorname{diam}\left(D_{\alpha \beta}\right)\left\|\nabla \omega_{h}\right\|_{0, D_{\alpha \beta}} \leq C h\left\|\nabla \omega_{h}\right\|_{0, D_{\alpha \beta}}
$$

Thus, we have

$$
\left\|\omega_{h}-\omega_{h}^{*}\right\|_{0, D_{j}} \leq C h\left\|\nabla \omega_{h}\right\|_{0, D_{j}} .
$$

The same reasoning can be held to bound $\left\|\omega_{h}-\omega_{h}^{*}\right\|_{0, D_{j}}$ on boundary diamond-cells by splitting $D_{j}$ into $D_{j, 1}^{\prime} \cup D_{j, 2}^{\prime}$. The result is obtained by summing (85) over the diamond-cells. 




Figure 10. Partition of diamond-cells.

We proceed then like in [1]. We again divide each diamond-cell $D_{j}$ either into $D_{j, 1} \cup D_{j, 2}$, or into $D_{j, 1}^{\prime} \cup D_{j, 2}^{\prime}$. Note that this choice is local to $D_{j}$ and does not influence the choice which can be made for the division of $D_{j^{\prime}}$, for $j^{\prime} \neq j$. We adopt to simplify the writing $\mathcal{T}_{j, \alpha}$ to represent $D_{j, \alpha}$ or $D_{j, \alpha}^{\prime}$. Further, we define $R T(\nabla \hat{\phi})$, the Raviart-Thomas interpolation of $\nabla \hat{\phi}$ on each $\mathcal{T}_{j, \alpha}$ (see [22]) by

$$
R T(\nabla \hat{\phi})_{\mid \mathcal{T}_{j, \alpha}} \in\left(P_{0}\left(\mathcal{T}_{j, \alpha}\right)\right)^{2} \oplus\left(\begin{array}{l}
x \\
y
\end{array}\right) P_{0}\left(\mathcal{T}_{j, \alpha}\right)
$$

and

$$
\int_{s} R T(\nabla \hat{\phi}) \cdot \mathbf{n} \mathrm{d} \xi=\int_{s} \nabla \hat{\phi} \cdot \mathbf{n} \mathrm{d} \xi
$$

for any side $s$ of $\mathcal{T}_{j, \alpha}$. We can state the following lemma:

Lemma 5.17. Let $\kappa \in \mathrm{H}_{0}^{1}(\Omega) \cap \mathrm{H}^{2}(\Omega), \mu:=-\Delta \kappa$ and let $\lambda \in \mathrm{H}_{0}^{1}(\Omega)+V_{h}$. Then if all diamond-cells are convex

$$
a_{h}(\kappa, \lambda)-(\mu, \lambda)_{\Omega}=\sum_{j \in[1, J]} \sum_{\alpha=1}^{2} \int_{\mathcal{T}_{j, \alpha}}\left[(\nabla \kappa-R T(\nabla \kappa)) \cdot \nabla \lambda+\mu\left(\langle\lambda\rangle_{j, \alpha}-\lambda\right)\right] \mathrm{d} \mathbf{x},
$$

where $\langle\lambda\rangle_{j, \alpha}$ is the average value of $\lambda$ over $\mathcal{T}_{j, \alpha}$.

Proof. By definition, $R T(\nabla \kappa) \cdot \mathbf{n}$ is a constant on each side of $\mathcal{T}_{j, \alpha}$. In addition, on two neighboring triangles $\mathcal{T}_{j, \alpha}$, the values of $R T(\nabla \kappa) \cdot \mathbf{n}$ on both sides of their common face are opposite one to the other, because of the orientation of the normal vector $\mathbf{n}$. By noting $\mathcal{S}$ the set of all the sides of all the $\mathcal{T}_{j, \alpha}$ and $\mathbf{n}_{s}$ the normal unit vector to a side $s$ in $\mathcal{S}$, and $[\lambda]_{s}$ the jump of $\lambda$ through $s$, then

$$
\sum_{j \in[1, J]} \sum_{\alpha=1}^{2} \int_{\partial \mathcal{T}_{j, \alpha}} R T(\nabla \kappa) \cdot \mathbf{n} \lambda \mathrm{d} \xi=\sum_{s \in \mathcal{S}, s \not \subset \Gamma} R T(\nabla \kappa) \cdot \mathbf{n} \int_{s}[\lambda]_{s} \mathrm{~d} \xi+\sum_{s \in \mathcal{S}, s \subset \Gamma} R T(\nabla \kappa) \cdot \mathbf{n} \int_{s} \lambda \mathrm{d} \xi .
$$

If $\lambda$ is in $\mathrm{H}_{0}^{1}(\Omega)$, then $[\lambda]_{s}=0, \forall s \in \mathcal{S}, s \not \subset \Gamma$ and $\lambda$ vanishes on $\Gamma$. Therefore,

$$
\sum_{j \in[1, J]} \sum_{\alpha=1}^{2} \int_{\partial \mathcal{T}_{j, \alpha}} R T(\nabla \kappa) \cdot \mathbf{n} \lambda \mathrm{d} \xi=0 .
$$


In addition, if $\lambda$ is in $V_{h}$, then $[\lambda]_{s}$ is a polynomial of degree one, which vanishes at the midpoint of $s$ (by construction of the functions of $V_{h}$ ). Its integral on $s$ is thus null. In addition, $\lambda$ vanishes on $\Gamma$. Thus, equality (87) remains true if $\lambda \in V_{h}$. But we can also write equality (87) in the following way

$$
\sum_{j \in[1, J]} \sum_{\alpha=1}^{2}\left(\int_{\mathcal{T}_{j, \alpha}} \nabla \cdot(R T(\nabla \kappa)) \lambda \mathrm{d} \mathbf{x}+\int_{\mathcal{T}_{j, \alpha}} R T(\nabla \kappa) \cdot \nabla \lambda \mathrm{d} \mathbf{x}\right)=0 .
$$

By subtracting this equality from $a_{h}(\kappa, \lambda)$, we obtain

$$
a_{h}(\kappa, \lambda)=\sum_{j \in[1, J]} \sum_{\alpha=1}^{2} \int_{\mathcal{T}_{j, \alpha}}(\nabla \kappa-R T(\nabla \kappa)) \cdot \nabla \lambda \mathrm{d} \mathbf{x}-\sum_{j \in[1, J]} \sum_{\alpha=1}^{2} \int_{\mathcal{T}_{j, \alpha}} \nabla \cdot(R T(\nabla \kappa)) \lambda \mathrm{d} \mathbf{x} .
$$

Let us note $\langle\lambda\rangle_{j, \alpha}$ the mean value of $\lambda$ on $\mathcal{T}_{j, \alpha}$. Since $\nabla \cdot(R T(\nabla \kappa))$ is by construction a constant on $\mathcal{T}_{j, \alpha}$, we may write the following series of equalities:

$$
\begin{array}{r}
\int_{\mathcal{T}_{j, \alpha}} \nabla \cdot(R T(\nabla \kappa)) \lambda \mathrm{d} \mathbf{x}=\langle\lambda\rangle_{j, \alpha} \int_{\mathcal{T}_{j, \alpha}} \nabla \cdot(R T(\nabla \kappa)) \mathrm{d} \mathbf{x}=\langle\lambda\rangle_{j, \alpha} \int_{\partial \mathcal{T}_{j, \alpha}} R T(\nabla \kappa) \cdot \mathbf{n} \mathrm{d} \xi \\
=\langle\lambda\rangle_{j, \alpha} \int_{\partial \mathcal{T}_{j, \alpha}} \nabla \kappa \cdot \mathbf{n} \mathrm{d} \xi=\langle\lambda\rangle_{j, \alpha} \int_{\mathcal{T}_{j, \alpha}} \Delta \kappa \mathrm{d} \mathbf{x}=-\langle\lambda\rangle_{j, \alpha} \int_{\mathcal{T}_{j, \alpha}} \mu \mathrm{d} \mathbf{x} .
\end{array}
$$

Equality (86) follows from (88) and (89).

The first term in the right-hand side of (82) can be bounded by the following lemma

Lemma 5.18. If all diamond-cells are convex and under Hypotheses 5.2 and 5.6, there exists a constant $C$ independent of the grid such that

$$
\left|a_{h}\left(\hat{\phi}, \omega_{h}\right)-\left(f, \omega_{h}\right)_{\Omega}\right| \leq C \frac{h}{\sin \tau^{*}}\left|\omega_{h}\right|_{1, h}|| f \mid \|_{0, \Omega}
$$

Proof. We apply Lemma 5.17 to $\kappa=\hat{\phi}, \mu=f$ and $\lambda=\omega_{h}$ to evaluate:

$$
a_{h}\left(\hat{\phi}, \omega_{h}\right)-\left(f, \omega_{h}\right)_{\Omega}=\sum_{j \in[1, J]} \sum_{\alpha=1}^{2} \int_{\mathcal{T}_{j, \alpha}}(\nabla \hat{\phi}-R T(\nabla \hat{\phi})) \cdot \nabla \omega_{h} \mathrm{~d} \mathbf{x}+\sum_{j \in[1, J]} \sum_{\alpha=1}^{2} \int_{\mathcal{T}_{j, \alpha}} f\left(\left\langle\omega_{h}\right\rangle_{j, \alpha}-\omega_{h}\right) \mathrm{d} \mathbf{x} .
$$

According to [1], Lemmas 4.1 and 4.2, we can state that under Hypothesis 5.6, there exists a constant $C$, independent of the grid such that

$$
\|\nabla \hat{\phi}-R T(\nabla \hat{\phi})\|_{0, \mathcal{T}_{j, \alpha}} \leq C \frac{h}{\sin \tau^{*}}|\hat{\phi}|_{2, \mathcal{T}_{j, \alpha}}
$$

Then, by a discrete Cauchy-Schwarz inequality, we have

$$
\sum_{j \in[1, J]} \sum_{\alpha=1}^{2} \int_{\mathcal{T}_{j, \alpha}}(\nabla \hat{\phi}-R T(\nabla \hat{\phi})) \cdot \nabla \omega_{h} \mathrm{~d} \mathbf{x} \leq C \frac{h}{\sin \tau^{*}}\left|\omega_{h}\right|_{1, h}|\hat{\phi}|_{2, \Omega}
$$

where the constant $C$ does not depend on $h$. Then, as the function $\left\langle\omega_{h}\right\rangle_{j, \alpha}-\omega_{h}$ has a vanishing mean-value on $\mathcal{T}_{j, \alpha}$, there exists a constant $C$, independent of $j, \alpha$ and $h$ such that

$$
\left\|\left\langle\omega_{h}\right\rangle_{j, \alpha}-\omega_{h}\right\|_{0, \mathcal{T}_{j, \alpha}} \leq C h\left|\omega_{h}\right|_{1, \mathcal{T}_{j, \alpha}} .
$$


Thus, we can write

$$
\sum_{j \in[1, J]} \sum_{\alpha=1}^{2} \int_{\mathcal{T}_{j, \alpha}} f\left(\left\langle\omega_{h}\right\rangle_{j, \alpha}-\omega_{h}\right) \mathrm{d} \mathbf{x} \leq C h\left|\omega_{h}\right|_{1, h}|| f \|_{0, \Omega},
$$

where the constant $C$ does not depend on $h$. The result follows from (91), (93), (37) and (94).

We end the consistency error estimation with

Proposition 5.19. If all diamond-cells are convex and under Hypotheses 5.2 and 5.6, there exists a constant $C$, independent of the grid such that

$$
\sup _{\omega_{h} \in V_{h}} \frac{\left|a_{h}\left(\phi, \omega_{h}\right)-\ell\left(\omega_{h}\right)\right|}{\left|\omega_{h}\right|_{1, h}} \leq C \frac{h}{\sin \tau^{*}}\|f\|_{0, \Omega} .
$$

Proof. The result follows from (82), (83) and (90).

In conclusion of Section 5.3, estimates (73), (74) or (75), and (95) allow us to state the following theorem:

Theorem 5.20. If all diamond-cells are convex and under Hypotheses 5.2 and 5.6, there exists a constant $C\left(\tau^{*}\right)$ independent of the grid such that

$$
\left|\hat{\phi}-\phi_{h}\right|_{1, h} \leq C\left(\tau^{*}\right) h\|f\|_{0, \Omega}
$$

Moreover, under Hypotheses 5.3 and 5.6, there exists a constant $C$ independent of the grid such that

$$
\left|\hat{\phi}-\phi_{h}\right|_{1, h} \leq C \frac{h}{\sin \tau^{*}}\left(\|f\|_{0, \Omega}+h\|f\|_{1, \Omega}\right) .
$$

Remark 5.21. In the case of Neumann homogeneous boundary conditions, a similar theorem holds. The key is that both (72) (with a modified definition of $V_{h}$ ) and Lemma 5.17 (with $\kappa \in \mathrm{H}^{1}(\Omega) \cap \mathrm{H}^{2}(\Omega), \nabla \kappa \cdot \mathbf{n}=0$ on $\Gamma$ and $\left.\lambda \in \mathrm{H}^{1}(\Omega)+V_{h}\right)$ remain true. The cases of non-homogeneous and of Robin boundary conditions present some additional error terms linked to the boundary and are not considered here.

\subsection{Discussion}

In Section 5.2, we obtained an order $h$ error estimate independently of the grid, under Hypothesis 5.5, while in Section 5.3, we obtained the same type of estimate under the weaker Hypothesis 5.6. However, an elementary calculation proves that these two estimates are true under the weakest hypothesis. Indeed,

$$
|\varepsilon|_{1, D}=\|\left.\nabla_{h}(\phi-\Pi \hat{\phi})\right|_{0, D}=\left|\phi_{h}-(\Pi \hat{\phi})_{h}\right|_{1, h} \leq\left|\phi_{h}-\hat{\phi}\right|_{1, h}+\left|\hat{\phi}-(\Pi \hat{\phi})_{h}\right|_{1, h}
$$

Estimates (74) and (96) (respectively (75) and (97)), which were all obtained under the weakest hypothesis (Hypothesis 5.6) allow to conclude that finite volume error estimates (70) (resp. (71)) are in fact true under this hypothesis (and replacing $\theta^{*}$ by $\tau^{*}$ ). It thus seems that the estimates obtained in Section 5.2 are not optimal with respect to the geometry of the diamond-cells.

\subsection{The case of non-convex diamond-cells}

When a diamond-cell $D_{j}$ is not convex, we can always divide it into $D_{j, 1} \cup D_{j, 2}$. On the other hand, splitting it into $D_{j, 1}^{\prime} \cup D_{j, 2}^{\prime}$ is not possible any more. On the contrary, we have

$$
D_{j}=D_{j, \alpha_{1}}^{\prime} \backslash D_{j, \alpha_{2}}^{\prime}
$$

with $\left(\alpha_{1}, \alpha_{2}\right)=(1,2)$ or $\left(\alpha_{1}, \alpha_{2}\right)=(2,1)$. In other words, $D_{j, \alpha_{1}}^{\prime}$ is the convex hull of the cell $D_{j}$, and $D_{j, \alpha_{2}}^{\prime}$ is the part of this convex hull that is not included in $D_{j}$. Such a diamond-cell is displayed in Figure 11 in 


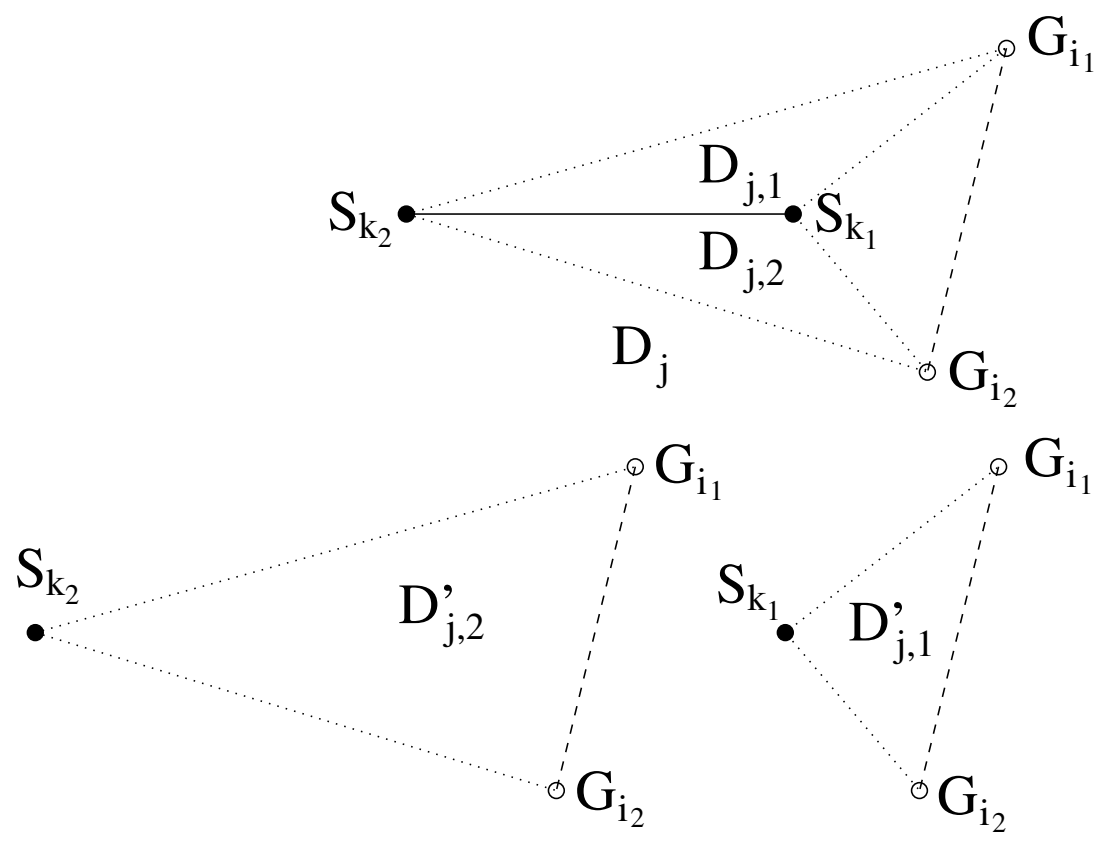

Figure 11. Non-convex diamond-cell.

the case $\left(\alpha_{1}, \alpha_{2}\right)=(2,1)$. Moreover, if the diamond-cell is not convex, Hypothesis 5.6 is not a consequence of Hypothesis 5.5 any more.

In order to obtain error estimates, we shall make the following supplementary hypotheses.

Hypothesis 5.22. There exists a constant $C$, independent of the mesh, such that

$$
\sum_{j \in[1, J]}|\hat{\phi}|_{2, D_{j, \alpha_{2}}^{\prime}}^{2} \leq C .
$$

Hypothesis 5.23. There exists a constant $C$, independent of the grid such that for any non-convex diamondcell $D_{j}$, and for $\alpha \in\{1 ; 2\}$,

$$
\frac{\left|D_{j, \alpha}^{\prime}\right|}{\left|D_{j}\right|} \leq C
$$

Remark 5.24. The condition (99) is trivially checked if the cells $D_{j, \alpha_{2}}^{\prime}$ do not overlap because in this case

$$
\sum_{j \in[1, J]}|\hat{\phi}|_{2, D_{j, \alpha_{2}}^{\prime}}^{2} \leq|\hat{\phi}|_{2, \Omega}^{2} \leq\|f\|_{0, \Omega}
$$

On the other hand, this condition may be violated if the grid is refined so that the same open set (dependent on $h$ ) is included in a growing (with $h$ ) number of $D_{j, \alpha_{2}}^{\prime}$.

We state the following result

Proposition 5.25. Under Hypotheses 5.2, 5.5, 5.6, 5.22 and 5.23, there exists a constant $C$, independent of the grid such that

$$
|\varepsilon|_{1, D} \leq C h .
$$


Proof. Let us reconsider the finite volume approach for the error estimate (Sect. 5.2). As regards the first term in (59), the bounds (60) and (64) remain valid, but we explicitly used the convexity of the diamond-cells in the estimate (65). Calculations are then modified in the following way:

$$
\int_{D_{j}}\left(\mathbf{e}_{j} \cdot \mathbf{n}_{j}^{\prime}\right)^{2} \mathrm{~d} \mathbf{x}=\left\|\mathbf{e}_{j} \cdot \mathbf{n}_{j}^{\prime}\right\|_{0, D_{j, \alpha_{1}}^{\prime}}^{2}-\left\|\mathbf{e}_{j} \cdot \mathbf{n}_{j}^{\prime}\right\|_{0, D_{j, \alpha_{2}}^{\prime}}^{2} \leq\left\|\mathbf{e}_{j} \cdot \mathbf{n}_{j}^{\prime}\right\|_{0, D_{j, \alpha_{1}}^{\prime}}^{2}
$$

Just like in Section 5.2, we know that there exists a constant $C$, independent of $j, \alpha$ and $h$, such that

$$
\left\|\mathbf{e}_{j} \cdot \mathbf{n}_{j}^{\prime}\right\|_{0, D_{j, \alpha_{1}}^{\prime}}^{2} \leq C h^{2}\left|\mathbf{e}_{j} \cdot \mathbf{n}_{j}^{\prime}\right|_{1, D_{j, \alpha_{1}}^{\prime}}^{2} \leq C h^{2}|\hat{\phi}|_{2, D_{j, \alpha_{1}}^{\prime}}^{2}=C h^{2}\left(|\hat{\phi}|_{2, D_{j}}^{2}+|\hat{\phi}|_{2, D_{j, \alpha_{2}}^{\prime}}^{2}\right)
$$

Thus, by summation over the diamond-cells, and under Hypothesis 5.22, there exists a constant $C$, independent of the grid such that

$$
\sum_{j \in[1, J]} \int_{D_{j}}\left(\mathbf{e}_{j} \cdot \mathbf{n}_{j}^{\prime}\right)^{2} \leq C h^{2}\left(\|f\|_{0, \Omega}+C\right) .
$$

Finally, under Hypothesis 5.5, we can prove the existence of a constant $C$, independent of the grid, such that

$$
\left(\sum_{j \in[1, J]} \int_{D_{j}}\left|\mathbf{e}_{j}\right|^{2}(\mathbf{x}) \mathrm{d} \mathbf{x}\right)^{1 / 2} \leq C h|\varepsilon|_{1, D} .
$$

As regards the second term in (59), the splitting of $D_{j}$ into $D_{j, 1} \cup D_{j, 2}$ is always possible, which makes it possible to leave this part of demonstration unchanged. Nevertheless, if the maximum angle of this couple of triangles approaches $\pi$, it can be judicious to reconsider the calculations by considering the splitting (98). Then, because $\left(\nabla_{h} \varepsilon\right)_{j}$ is a constant, we obtain

$$
\int_{D_{j}}\left(\nabla \hat{\phi}-\left(\nabla_{h} \Pi \hat{\phi}\right)_{j}\right) \cdot\left(\nabla_{h} \varepsilon\right)_{j} \mathrm{~d} \mathbf{x}=\left(\int_{D_{j, \alpha_{1}}^{\prime}}\left(\nabla \hat{\phi}-\nabla \omega_{j, \alpha_{1}}\right)(\mathbf{x}) \mathrm{d} \mathbf{x}-\int_{D_{j, \alpha_{2}}^{\prime}}\left(\nabla \hat{\phi}-\nabla \omega_{j, \alpha_{2}}\right)(\mathbf{x}) \mathrm{d} \mathbf{x}\right) \cdot\left(\nabla_{h} \varepsilon\right)_{j}
$$

By application of the Cauchy-Schwarz inequality on $D_{j, \alpha_{1}}^{\prime}$ and $D_{j, \alpha_{2}}^{\prime}$

$$
\left|\int_{D_{j}}\left(\nabla \hat{\phi}-\left(\nabla_{h} \Pi \hat{\phi}\right)_{j}\right) \cdot\left(\nabla_{h} \varepsilon\right)_{j} \mathrm{~d} \mathbf{x}\right| \leq\left(\sum_{\alpha=1}^{2}\left(\frac{\left|D_{j, \alpha}^{\prime}\right|}{\left|D_{j}\right|}\right)^{1 / 2}\left|\hat{\phi}-\omega_{j, \alpha}\right|_{1, D_{j, \alpha}^{\prime}}\right)\left|D_{j}\right|^{1 / 2}\left|\nabla_{h} \varepsilon\right|_{j}
$$

As previously, under Hypothesis 5.6, there exists a constant $C$ independent of $j, \alpha$ and $h$ such that

$$
\left|\hat{\phi}-\omega_{j, \alpha}\right|_{1, D_{j, \alpha}^{\prime}} \leq C h|\hat{\phi}|_{2, D_{j, \alpha}^{\prime}}
$$

Then, thanks to Hypothesis 5.23,

$$
\left|\int_{D_{j}}\left(\nabla \hat{\phi}-\left(\nabla_{h} \Pi \hat{\phi}\right)_{j}\right) \cdot\left(\nabla_{h} \varepsilon\right)_{j} \mathrm{~d} \mathbf{x}\right| \leq C h\left(|\hat{\phi}|_{2, D_{j, \alpha_{1}}^{\prime}}+|\hat{\phi}|_{2, D_{j, \alpha_{2}}^{\prime}}\right)\left|D_{j}\right|^{1 / 2}\left|\nabla_{h} \varepsilon\right|_{j} .
$$

By application of the discrete Cauchy-Schwarz inequality, and under Hypothesis 5.22, the estimate

$$
\sum_{j \in[1, J]} \int_{D_{j}}\left(\nabla \hat{\phi}-\left(\nabla_{h} \Pi \hat{\phi}\right)_{j}\right) \cdot\left(\nabla_{h} \varepsilon\right)_{j} \mathrm{~d} \mathbf{x} \leq C h|\varepsilon|_{1, D}
$$


remains valid with a constant $C$ which does not depend on the grid.

The result of the proposition follows from (59), (100) and (101).

Remark 5.26. Finding a criterion to check that a given family of meshes verifies Hypotheses 5.22 and 5.23 is beyond the scope of this paper.

\section{ERror estimates IN THE L ${ }^{2}$ NORM}

In this part, we study the error in the $\mathrm{L}^{2}$ norm between the exact solution $\hat{\phi}$ and the function $\phi_{h}=L(\phi)$ built from the element $\phi \in V$ which is solution of the system (11)-(14). For the sake of simplicity, we shall suppose in this part that all diamond-cells are convex. Although the study was not performed for non-convex diamond-cells, the numerical results given in [15] for very distorted meshes which contain non-convex diamond cells show that convergence in the $L^{2}$ norm also takes place for such meshes.

We use the traditional Aubin-Nitsche duality argument to write

$$
\left\|\phi_{h}-\hat{\phi}\right\|_{0, \Omega}=\sup _{v \in \mathrm{L}^{2}(\Omega)} \frac{\left|\left(\phi_{h}-\hat{\phi}, v\right)_{\Omega}\right|}{\|v\|_{0, \Omega}}
$$

For a given $v \in \mathrm{L}^{2}(\Omega)$ let us consider $\psi$, solution of the problem

$$
\begin{array}{rlll}
-\Delta \psi=v & \text { in } & \Omega \\
\psi=0 & \text { on } & \Gamma .
\end{array}
$$

The domain $\Omega$ being polygonal convex, we know that $\psi$ belongs to $\mathrm{H}_{0}^{1}(\Omega) \cap \mathrm{H}^{2}(\Omega)$, and that there exists a constant $K$ such that

$$
\|\psi\|_{2, \Omega} \leq K\|v\|_{0, \Omega} .
$$

In order to evaluate $\left(\phi_{h}-\hat{\phi}, v\right)_{\Omega}$, we start by proving a useful lemma:

Lemma 6.1. Let $\kappa \in \mathrm{H}_{0}^{1}(\Omega) \cap \mathrm{H}^{2}(\Omega)$ and $\mu:=-\Delta \kappa$; let $\omega \in \mathrm{H}_{0}^{1}(\Omega) \cap \mathrm{H}^{2}(\Omega)$ and let $\omega_{h} \in V_{h}$. Then, if all diamond-cells are convex,

$$
a_{h}\left(\kappa, \omega_{h}\right)-\left(\mu, \omega_{h}\right)_{\Omega}=\sum_{j \in[1, J]} \sum_{\alpha=1}^{2} \int_{\mathcal{T}_{j, \alpha}}\left\{(\nabla \kappa-R T(\nabla \kappa)) \cdot \nabla\left(\omega_{h}-\omega\right)+\mu\left[\left(\omega-\omega_{h}\right)-\left\langle\omega-\omega_{h}\right\rangle_{j, \alpha}\right]\right\} \mathrm{d} \mathbf{x} .
$$

Proof. Applying lemma 5.17 to $\kappa$ and $\omega_{h}$ on the one hand and to $\kappa$ and $\omega$ on the other hand, we obtain respectively

$$
a_{h}\left(\kappa, \omega_{h}\right)-\left(\mu, \omega_{h}\right)_{\Omega}=\sum_{j \in[1, J]} \sum_{\alpha=1}^{2} \int_{\mathcal{T}_{j, \alpha}}\left[(\nabla \kappa-R T(\nabla \kappa)) \cdot \nabla \omega_{h}+\mu\left(\left\langle\omega_{h}\right\rangle_{j, \alpha}-\omega_{h}\right)\right] \mathrm{d} \mathbf{x}
$$

and

$$
a_{h}(\kappa, \omega)-(\mu, \omega)_{\Omega}=\sum_{j \in[1, J]} \sum_{\alpha=1}^{2} \int_{\mathcal{T}_{j, \alpha}}(\nabla \kappa-R T(\nabla \kappa)) \cdot \nabla \omega \mathrm{d} \mathbf{x}+\sum_{j \in[1, J]} \sum_{\alpha=1}^{2} \int_{\mathcal{T}_{j, \alpha}} \mu\left(\langle\omega\rangle_{j, \alpha}-\omega\right) \mathrm{d} \mathbf{x} .
$$

But of course

$$
a_{h}(\kappa, \omega)-(\mu, \omega)_{\Omega}=\sum_{j \in[1, J]} \sum_{\alpha=1}^{2} \int_{\mathcal{T}_{j, \alpha}}(\nabla \kappa \cdot \nabla \omega-\mu \omega) \mathrm{d} \mathbf{x}=\int_{\Omega}(\nabla \kappa \cdot \nabla \omega-\mu \omega) \mathrm{d} \mathbf{x}=0 .
$$


The result (104) is obtained by subtracting (106) to (105).

Proposition 6.2. Let $\psi_{h} \in V_{h}$. The following equality holds if all diamond-cells are convex

$$
\begin{aligned}
\left(\phi_{h}-\hat{\phi}, v\right)_{\Omega} & =a_{h}\left(\phi_{h}-\hat{\phi}, \psi-\psi_{h}\right) \\
& -\sum_{j \in[1, J]} \sum_{\alpha=1}^{2} \int_{\mathcal{T}_{j, \alpha}}(\nabla \hat{\phi}-R T(\nabla \hat{\phi})) \cdot \nabla\left(\psi_{h}-\psi\right) \mathrm{d} \mathbf{x} \\
& -\sum_{j \in[1, J]} \sum_{\alpha=1}^{2} \int_{\mathcal{T}_{j, \alpha}} f\left[\left(\psi-\psi_{h}\right)-\left\langle\psi-\psi_{h}\right\rangle_{j, \alpha}\right] \mathrm{d} \mathbf{x} \\
& -\sum_{j \in[1, J]} \sum_{\alpha=1}^{2} \int_{\mathcal{T}_{j, \alpha}}(\nabla \psi-R T(\nabla \psi)) \cdot \nabla\left(\phi_{h}-\hat{\phi}\right) \mathrm{d} \mathbf{x} \\
& -\sum_{j \in[1, J]} \sum_{\alpha=1}^{2} \int_{\mathcal{T}_{j, \alpha}} v\left[\left(\hat{\phi}-\phi_{h}\right)-\left\langle\hat{\phi}-\phi_{h}\right\rangle_{j, \alpha}\right] \mathrm{d} \mathbf{x} \\
& +\sum_{j} \int_{D_{j}} f\left(\psi_{h}^{*}-\psi_{h}\right) \mathrm{d} \mathbf{x} .
\end{aligned}
$$

Proof. For any $\psi_{h} \in V_{h}$, there holds

$$
\begin{aligned}
\left(\phi_{h}-\hat{\phi}, v\right)_{\Omega} & =\sum_{j} \int_{D_{j}}\left(\phi_{h}-\hat{\phi}\right)(-\Delta \psi) \mathrm{d} \mathbf{x}=a_{h}\left(\phi_{h}-\hat{\phi}, \psi\right)-\sum_{j} \int_{\partial D_{j}} \phi_{h} \nabla \psi \cdot \mathbf{n} \mathrm{d} \xi \\
& =a_{h}\left(\phi_{h}-\hat{\phi}, \psi-\psi_{h}\right)+a_{h}\left(\phi_{h}-\hat{\phi}, \psi_{h}\right)-\sum_{j} \int_{\partial D_{j}} \phi_{h} \nabla \psi \cdot \mathbf{n} \mathrm{d} \xi
\end{aligned}
$$

Thanks to (34), we have

$$
a_{h}\left(\phi_{h}-\hat{\phi}, \psi_{h}\right)=\ell\left(\psi_{h}\right)-a_{h}\left(\hat{\phi}, \psi_{h}\right)=\left(f, \psi_{h}^{*}\right)_{\Omega}-a_{h}\left(\hat{\phi}, \psi_{h}\right)=\left(f, \psi_{h}^{*}-\psi_{h}\right)_{\Omega}-\left[a_{h}\left(\hat{\phi}, \psi_{h}\right)-\left(f, \psi_{h}\right)_{\Omega}\right]
$$

In addition, the last term in (108) can be evaluated in the following way

$$
\sum_{j} \int_{\partial D_{j}} \phi_{h} \nabla \psi \cdot \mathbf{n} \mathrm{d} \xi=\sum_{j} \int_{D_{j}}\left[\nabla \phi_{h} \cdot \nabla \psi-(-\Delta \psi) \phi_{h}\right] \mathrm{d} \mathbf{x}=a_{h}\left(\psi, \phi_{h}\right)-\left(v, \phi_{h}\right)_{\Omega}
$$

We thus have

$$
\left(\phi_{h}-\hat{\phi}, v\right)_{\Omega}=a_{h}\left(\phi_{h}-\hat{\phi}, \psi-\psi_{h}\right)-\left[a_{h}\left(\hat{\phi}, \psi_{h}\right)-\left(f, \psi_{h}\right)_{\Omega}\right]-\left[a_{h}\left(\psi, \phi_{h}\right)-\left(v, \phi_{h}\right)_{\Omega}\right]+\left(f, \psi_{h}^{*}-\psi_{h}\right)_{\Omega}
$$

Applying lemma 6.1 for $\kappa=\hat{\phi}, \mu=f, \omega=\psi$ and $\omega_{h}=\psi_{h}$ on the one hand and for $\kappa=\psi, \mu=\nu, \omega=\hat{\phi}$ and $\omega_{h}=\phi_{h}$ on the other hand leads to the announced result.

This leads to the following result:

Theorem 6.3. If all diamond-cells are convex and under Hypotheses 5.2 and 5.6, there exists a constant C, depending only on $\tau^{*}$, such that

$$
\left\|\phi_{h}-\hat{\phi}\right\|_{0, \Omega} \leq C\left(\tau^{*}\right) h\|f\|_{0, \Omega}
$$


Proof. The first term in the right-hand side of (107) can be bounded by

$$
\left|\phi_{h}-\hat{\phi}\right|_{1, h}\left|\psi-\psi_{h}\right|_{1, h}
$$

Then, thanks to estimate (92), the second term in the right-hand side of (107) can be bounded by

$$
C h|\hat{\phi}|_{2, \Omega}\left|\psi-\psi_{h}\right|_{1, h}
$$

where, under Hypothese 5.6, the constant $C$ depends only on $\tau^{*}$. Similarly, the fourth term can be bounded by

$$
C h|\psi|_{2, \Omega}\left|\hat{\phi}-\phi_{h}\right|_{1, h} .
$$

In addition, on each triangle $\mathcal{T}_{j, \alpha}$, the function $\left(\psi-\psi_{h}\right)-\left\langle\psi-\psi_{h}\right\rangle_{j, \alpha}$ has a vanishing mean-value, which implies the existence of a constant $C$, independent of the grid, such that

$$
\left\|\left(\psi-\psi_{h}\right)-\left\langle\psi-\psi_{h}\right\rangle_{j, \alpha}\right\|_{0, \mathcal{T}_{j, \alpha}} \leq C \operatorname{diam}\left(\mathcal{T}_{j, \alpha}\right)\left|\psi-\psi_{h}\right|_{1, \mathcal{T}_{j, \alpha}} \leq C h\left|\psi-\psi_{h}\right|_{1, \mathcal{T}_{j, \alpha}} .
$$

From this, we deduce that the third term in the right-hand side of (107) is bounded by

$$
C h\left|\psi-\psi_{h}\right|_{1, h}\|f\|_{0, \Omega} .
$$

In the same way, the fifth term in the right-hand side of (107) can be bounded by

$$
C h\left|\hat{\phi}-\phi_{h}\right|_{1, h}\|v\|_{0, \Omega} .
$$

Taking into account these various results, we obtain

$$
\begin{aligned}
\left|\left(\phi_{h}-\hat{\phi}, v\right)\right| & \leq C h\left|\phi_{h}-\hat{\phi}\right|_{1, h}\left(|\psi|_{2, \Omega}+\|v\|_{0, \Omega}\right) \\
& +\left|\psi-\psi_{h}\right|_{1, h}\left[\left|\phi_{h}-\hat{\phi}\right|_{1, h}+C h\left(|\hat{\phi}|_{2, \Omega}+\|f\|_{0, \Omega}\right)\right]+\left|\sum_{j} \int_{D_{j}} f\left(\psi_{h}^{*}-\psi_{h}\right) \mathrm{d} \mathbf{x}\right|,
\end{aligned}
$$

where the constants depend only on $\tau^{*}$.

Inequality (96) allows to bound the first term in the right-hand side of (109). In addition, if we choose $\psi_{h}:=L(\Pi \psi)$ (see Defs. 4.2 and 5.4), we can write, thanks to the calculations carried out in Section 5.3.1,

$$
\left|\psi-\psi_{h}\right|_{1, h} \leq C h|\psi|_{2, \Omega}
$$

with a constant $C$ which, under Hypothesis 5.6, depends only on $\tau^{*}$. Then, thanks to (103) and (37)

$$
\left|\left(\phi_{h}-\hat{\phi}, v\right)\right| \leq C h^{2}\|f\|_{0, \Omega}\|v\|_{0, \Omega}+\left|\sum_{j} \int_{D_{j}} f\left(\psi_{h}^{*}-\psi_{h}\right) \mathrm{d} \mathbf{x}\right| .
$$

A bound of the second term in the right-hand side of (110) was obtained in Section 5.3.2, (formula (83)). We can transform this inequality into

$$
\left|\left(f, \psi_{h}^{*}-\psi_{h}\right)_{\Omega}\right| \leq\left. C h|| f\right|_{0, \Omega}\left(|\psi|_{1, \Omega}+\left|\psi-\psi_{h}\right|_{1, h}\right) \leq C h|| f\left\|_{0, \Omega}\left(\|\psi\|_{2, \Omega}(1+C h)\right) \leq C h|| f\right\|_{0, \Omega}\|v\|_{0, \Omega}
$$

with a constant $C$ depending only on $\tau^{*}$. The result of the theorem follows from (102), (110) and (111). 
Remark 6.4. The result stated by Theorem 6.3 is only first order, like that obtained through a discrete Poincaré inequality in [9]. Whether our result could have been obtained through this type of technique is an open question. The first-order term is linked to the finite element formulation (34)-(35) which displays in the definition of $\ell$ the non-traditional term $\left(f, \psi_{h}^{*}\right)_{\Omega}$ instead of the more traditional term $\left(f, \psi_{h}\right)_{\Omega}$. On the other hand, the numerical results presented in Section 8 tend to show that this error is in fact second-order. Moreover, we can prove (almost) second-order in the $\mathrm{L}^{2}$ norm in the situation of the next section.

\section{ERROR ESTIMATES ON HOMOTHETICALLY REFINED MESHES}

In this section, we consider a family of meshes refined in the following way. We choose a coarse triangular grid of the domain $\Omega$. Then, each one of the coarse triangles is divided into four similar sub-triangles, which are obtained by joining the midpoints of the sides of the coarse triangle, as indicated in Figure 12. Then, this process is repeated to obtain finer and finer grids. On such families, we always choose the points $G_{i}$ to be the barycenters of the cells $T_{i}$. These meshes will be called homothetically refined. We only sketch the proofs of the results; details can be found in [8]. We shall further suppose that $f \in \mathrm{H}^{1}$ (Hyp. 5.3), so that $\hat{\phi} \in \mathrm{H}^{3}$.

Going back to (58) and applying a discrete Cauchy-Schwarz inequality leads to

$$
|\varepsilon|_{1, D}^{2} \leq \sum_{j}\left|D_{j}\right|\left|d_{j}(\hat{\phi})\right|^{2} \quad \text { with } \quad d_{j}(\hat{\phi})=\left(\delta \hat{\phi}-\nabla_{h} \Pi \hat{\phi}\right)_{j}
$$

The refined estimates are based on the distinction between two types of diamond-cells, whether they are associated to a side $A_{j}$ which is included in the boundaries of the triangles of the initial coarse mesh (we shall write that $j \in \mathcal{J}$ ) or not (we shall write $j \notin \mathcal{J}$ ). If $j \in \mathcal{J}$, then the local estimates presented in Sections 5.2 remain valid for the associated diamond-cell $D_{j}$ so that $\left|D_{j}\right|\left|d_{j}(\hat{\phi})\right|^{2} \leq C h^{2}|\hat{\phi}|_{2, D_{j}}^{2}$. As the total area of these cells is bounded by $C h$, it can be shown that for all $\alpha>0$, there exists a constant $C_{\alpha}$ such that

$$
\sum_{j \in \mathcal{J}}\left|D_{j}\right|\left|d_{j}(\hat{\phi})\right|^{2} \leq C_{\alpha} h^{3-2 \alpha}|| f \|_{1, \Omega}^{2} .
$$

Now if the side $A_{j}$ is not included in the boundaries of the triangles of the initial coarse mesh, then it is easily seen that the corresponding diamond-cell is a parallelogram. But if $D_{j}$ is a parallelogram, then the term $d_{j}(p)$ vanishes for any second-order polynomial $p$ and standard results of numerical analysis lead us to the conclusion that for these cells, $\left|D_{j}\right|\left|d_{j}(\hat{\phi})\right|^{2} \leq C h^{4}|\hat{\phi}|_{3, D_{j}}^{2}$, so that

$$
\sum_{j \notin \mathcal{J}}\left|D_{j}\right|\left|d_{j}(\hat{\phi})\right|^{2} \leq C h^{4}|\hat{\phi}|_{3, \Omega}^{2} \leq C h^{4}\|f\|_{1, \Omega}^{2}
$$

Summing (112) and (113) gives the superconvergence result:

Theorem 7.1. On a homothetically refined family of meshes, and under Hypothesis 5.3, and for all $\alpha$ (with $1 / 2 \geq \alpha>0)$, there exists a constant $C$ depending only on $\alpha$ and on the initial coarse mesh such that

$$
|\varepsilon|_{1, D} \leq C h^{3 / 2-\alpha}|| f \|_{1, \Omega} .
$$

As far as the error in the $\mathrm{L}^{2}$ norm is concerned, going back to Section 6 , the bound of the second term in (110) can be refined. There holds

$$
\int_{D_{j}} f\left(\psi_{h}^{*}-\psi_{h}\right) \mathrm{d} \mathbf{x}=\int_{D_{j}}\left(f-\langle f\rangle_{j}\right)\left(\psi_{h}^{*}-\psi_{h}\right) \mathrm{d} \mathbf{x}+\langle f\rangle_{j} \int_{D_{j}}\left(\psi_{h}^{*}-\psi_{h}\right) \mathrm{d} \mathbf{x}
$$




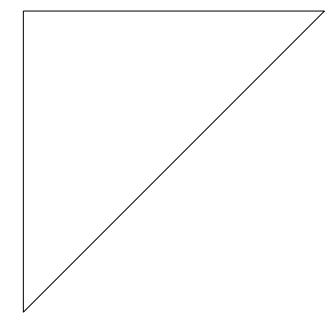

(a) coarse triangle



(b) refined triangle

FiguRE 12. Refinement of a triangle into four similar sub-triangles.

where for all $j,\langle f\rangle_{j}$ denotes the average value of $f$ over $D_{j}$. As $f$ belongs to $\mathrm{H}^{1}$, and thanks to results obtained in Section 5.3, the first term in the right-hand-side of (114) can be shown to be bounded by $C h^{2}\|f\|_{1, D_{j}}\|\psi\|_{2, D_{j}}$. On the other hand, the second term in the right-hand-side of (114) vanishes if the diamond-cell $D_{j}$ is a parallelogram and is bounded by $C h\left|D_{j}\right|\left|\langle f\rangle_{j}\right|\left|\nabla \psi_{h}\right|_{j}$ if not. As the total area of diamond-cells which are not parallelograms is bounded by $C h$, the following result holds:

Theorem 7.2. On a homothetically refined family of meshes, and under Hypothesis 5.3, for all $1 \geq \alpha>0$, there exists a constant $C$, depending only on $\alpha$ and on the initial coarse mesh, such that

$$
\left\|\phi_{h}-\hat{\phi}\right\|_{0, \Omega} \leq C h^{2-\alpha}\|f\|_{1, \Omega} .
$$

\section{Numerical RESUlts}

In all but last of the following tests, the computational domain is the unit square: $\Omega=[0 ; 1] \times[0 ; 1]$ and the right-hand-side and boundary conditions are chosen so that the exact solution $\hat{\phi}$ is given by

$$
\hat{\phi}(x, y)=x y \exp (x) \cos (\pi y) .
$$

We test the finite volume method on various types of grids and we define two types of discrete error in the $\mathrm{H}_{0}^{1}$ norm, corresponding respectively to the finite volume approach (Sect. 5.2) and to the finite element approach (Sect. 5.3):

$$
e 1_{F V}^{2}(h):=\frac{\sum_{j}\left|D_{j}\right|\left|\nabla_{h}(\phi-\Pi \hat{\phi})\right|_{j}^{2}}{\sum_{j}\left|D_{j}\right|\left|\nabla_{h}(\Pi \hat{\phi})\right|_{j}^{2}} \text { and } e 1_{F E}^{2}(h):=\frac{\sum_{j}\left|D_{j}\right|\left|\left(\nabla \phi_{h}\right)_{j}-\nabla \hat{\phi}\left(B_{j}\right)\right|^{2}}{\sum_{j}\left|D_{j}\right|\left|\nabla \hat{\phi}\left(B_{j}\right)\right|^{2}}
$$

where $\Pi \hat{\phi}$ is defined through (39)-(40) and where $\nabla \hat{\phi}\left(B_{j}\right)$ is the value of the gradient of the exact solution at the barycenter of $D_{j}$ (denoted by $B_{j}$ ). In addition, we define an error in the discrete $\mathrm{L}^{2}$ norm by

$$
e 0^{2}(h):=\frac{\frac{1}{2}\left(\sum_{i}\left|T_{i}\right|\left(\phi_{i}^{T}-(\Pi \hat{\phi})_{i}^{T}\right)^{2}+\sum_{k}\left|P_{k}\right|\left(\phi_{k}^{P}-(\Pi \hat{\phi})_{k}^{P}\right)^{2}\right)}{\frac{1}{2}\left(\sum_{i}\left|T_{i}\right|\left((\Pi \hat{\phi})_{i}^{T}\right)^{2}+\sum_{k}\left|P_{k}\right|\left((\Pi \hat{\phi})_{k}^{P}\right)^{2}\right)} .
$$





FIGURE 13. Unstructured triangular meshes.

We compare these errors with the errors obtained by using the standard $P^{1}$ Lagrange finite element method:

$$
e 1_{P 1}^{2}:=\frac{\sum_{i}\left|T_{i}\right|\left|\nabla \phi_{h}^{P 1}-\nabla \hat{\phi}\left(G_{i}\right)\right|_{i}^{2}}{\sum_{i}\left|T_{i}\right|\left|\nabla \hat{\phi}\left(G_{i}\right)\right|_{i}^{2}} \text { and } e 0_{P 1}^{2}:=\frac{\sum_{k}\left|P_{k}\right|\left|\phi_{h}^{P 1}\left(S_{k}\right)-(\Pi \hat{\phi})_{k}^{P}\right|^{2}}{\sum_{k}\left|P_{k}\right|\left|(\Pi \hat{\phi})_{k}^{P}\right|^{2}}
$$

where $\phi_{h}^{P 1}$ is the solution of the associated finite element problem. We also compare them with the errors obtained by using the standard four-point finite volume scheme (nicknamed "VF4") which was first studied in [14] in the case of triangles, and then extended to more general meshes and boundary conditions in [12]. Note that this scheme is well defined on triangular meshes fulfilling the strict Delaunay condition (see [9], Ex. 9.1) if one chooses the intersection of orthogonal bisectors (IOB) of the triangle edges as control points associated to the triangles. For triangular meshes that do not meet this condition, the VF4 scheme was extended and numerically studied in [4]. In that case, the matrix of the resulting linear system is not necessarily definite positive. This has the drawbacks that one cannot prove that the linear system has a unique solution, and that solving it by an iterative method is then more expensive than in the definite positive case. For the VF4 scheme, the discrete errors are defined by

$$
e 1_{V F 4}^{2}(h):=\frac{\sum_{j} \frac{\left|A_{j}\right|}{\left|A_{j}^{\prime}\right|}\left[(\phi-\Pi \hat{\phi})_{i_{1}(j)}-(\phi-\Pi \hat{\phi})_{i_{2}(j)}\right]^{2}}{\sum_{j} \frac{\left|A_{j}\right|}{\left|A_{j}^{\prime}\right|}\left[(\Pi \hat{\phi})_{i_{1}(j)}-(\Pi \hat{\phi})_{i_{2}(j)}\right]^{2}} \text { and } e 0_{V F 4}^{2}(h):=\frac{\sum_{i}\left|T_{i}\right|\left((\phi-\Pi \hat{\phi})_{i}\right)^{2}}{\sum_{i}\left|T_{i}\right|\left((\Pi \hat{\phi})_{i}\right)^{2}}
$$

Initially, we consider a family of six unstructured grids made up of increasingly small triangles. The first two of these grids are represented in Figure 13. The numerical errors in the $\mathrm{H}_{0}^{1}$ norms and in the $\mathrm{L}^{2}$ norms are presented in logarithmic scale respectively in Figures 14 and 15, on which we also respectively plotted a straight line of slope 1 and a line of slope 2 . We remark, as proved previously, a first-order convergence of the presented scheme, with the $e 1_{F E}$ error eight to ten times weaker than the $e 1_{P 1}$ error on a given grid, and twice weaker than the $e 1_{F V 4}$ error. Of course, on a given grid, the computation is longer by the scheme suggested here because it involves more unknowns than the Lagrange $P^{1}$ finite element method, or the VF4 scheme. As far as the $P^{1}$ finite element method is concerned, this matter was quantified in [8], where it was concluded that a fair comparison of the two methods for a fixed CPU time is obtained by comparing the error due to the finite volume approximation on a given grid, with that due to the Lagrange $P^{1}$ finite element approximation on the grid of higher refinement. The finite volume scheme thus obtains a precision four to five times better in the discrete $\mathrm{H}_{0}^{1}$ norm for a fixed CPU time. On the contrary, as the error curves in the discrete $\mathrm{L}^{2}$ norm almost overlap and are parallel to a line of slope 2 , it is possible to conclude that the precision obtained is approximately 4 times 


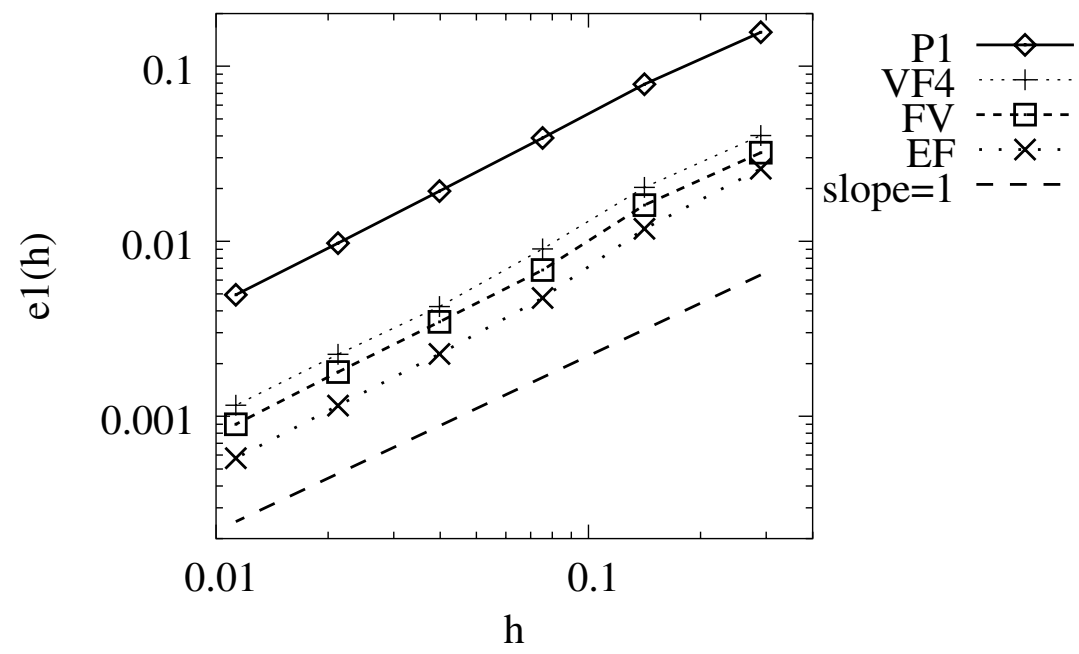

FiguRE 14. Convergence in the $\mathrm{H}_{0}^{1}$ norm on the family of unstructured triangular grids.

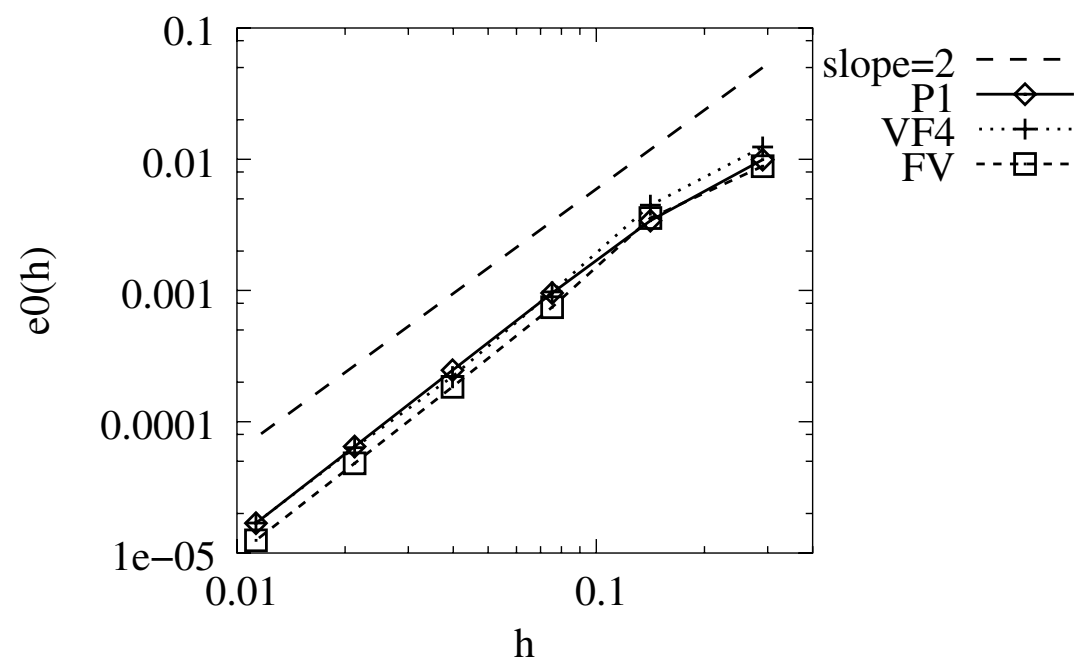

FiguRE 15. Convergence in the $\mathrm{L}^{2}$ norm on the family of unstructured triangular grids.

better in the $\mathrm{L}^{2}$ norm by the Lagrange finite element method than by the finite volume scheme presented here, for a fixed CPU time.

As far as the FV4 scheme is concerned, the comparison for a fixed CPU time greatly depends on the quality of the triangular mesh. Indeed, for the first three meshes of the family considered in the present example, only very few pairs of triangles did not meet the Delaunay condition, and the resolution by the conjugate gradient method of the linear system associated to the scheme was quite easy and led to a resolution around three times faster on a given mesh for the VF4 scheme than for the scheme presented in this paper; on the other hand, the situation became worse and worse for the last three meshes of the family (around a hundred pairs of triangles did not meet the Delaunay condition in the finest mesh), which led to resolutions of the linear systems which were more expensive for the VF4 scheme than for the scheme presented here, up to ten times longer on the finest mesh (however, in that case, the conjugate gradient was probably not the most efficient method to solve the linear system, but the comparison was performed for a given resolution method). 


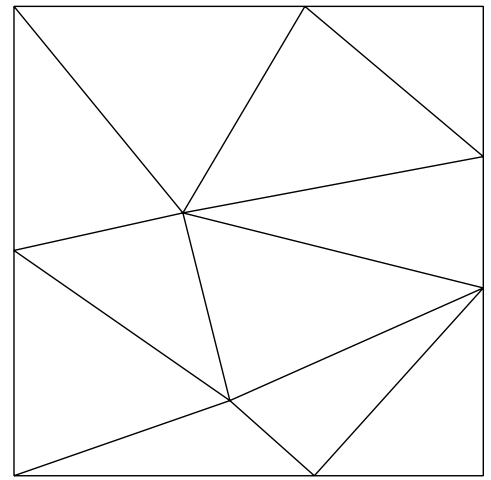

(a) Coarse mesh



(b) Refined mesh

FiguRE 16. Triangular meshes refined by subdivision.

Another point should be mentioned here: the method presented here computes both components of the gradients at the interfaces of the cells, and not only the normal component. There are cases where this is an important feature, although they are not considered in the present report. Anisotropic and/or non-linear diffusion is for example treated in [15]. We may also cite the case of groundwater flow, where the velocity $u$, defined by $u=-K \nabla \phi$ (with $K$ the permeability tensor), is a coefficient in the convection diffusion equation of the concentration of some chemical species. Models for this application usually consider diffusion tensors that require both components of $u$. We refer for example to [26] for a recent benchmark for the special case of radio-elements. An alternative to the computation of both components of the gradient is the method described in [10], which reconstructs the whole gradient from the knowledge of the normal components at the interfaces.

We conclude that on this type of grids, the method suggested here is useful if one is in fact interested in an accurate approximation of both components of the gradients of the solution at the interfaces of the cells.

As a second test, we consider a family of homothetically refined grids (see Sect. 7 for the definition). The first two of these grids are represented in Figure 16. The numerical errors in the $\mathrm{H}_{0}^{1}$ and $\mathrm{L}^{2}$ norms are presented in logarithmic scale respectively in Figures 17 and 18. In Figure 17 we also plotted two straight lines, one whose slope is 1 and the other whose slope is 1.5, and in Figure 18 a line whose slope is 2. We observe the superconvergence in the $\mathrm{H}_{0}^{1}$ norm predicted in Section 7 (order 1.5 whereas the polynomials are of order 1 ), whereas $P^{1}$ Lagrange finite elements continue to converge with the order 1 . We conclude that, as far as possible, the refinement of triangular grids should be performed by splitting the triangles into four similar sub-triangles. In the discrete $\mathrm{L}^{2}$ norm, the conclusions stated on the previous family of grids remain valid.

The third family is made up of grids of increasingly flat triangles built in the following way. Let $n$ be a non-zero integer. We divide $\Omega$ into $4^{n}$ horizontal stripes of the same height and we divide each one of these stripes into similar triangles (except those at both ends) so that there are $2^{n}$ bases of triangles in the width of a stripe and we choose $n \in[1 ; 6]_{\mathbb{N}}$. Figure 19 represents the first two of these grids. There holds $h=1 / 2^{n}$ and the greatest angle $\theta_{m}$ at the top of the triangles is such that $\tan \left(\theta_{m} / 2\right)=2^{n-1}$. The numerical errors in the $\mathrm{H}_{0}^{1}$ and $\mathrm{L}^{2}$ norms are presented in logarithmic scale respectively in Figures 22 and 23 . In Figure 22, we also displayed the curve $h / \sin \theta_{m}$ (named "P1 theor") which corresponds to the higher bound (up to a multiplicative constant) of the error estimates obtained by Jamet in [18]. We observe that this curve properly reproduces the behavior of the $e 1_{P 1}$ (the curves are almost parallel). We also plotted a straight line of slope 0.5 to which $e 1_{F V}$ is parallel and a line of slope 1.5 to which $e 1_{F E}$ is parallel.

For this type of grids, the diamond-cells are of several types, according to whether they are associated with interior diamond-cells (see Fig. 20) or they have a node located on the boundary (Fig. 21). In Figure 20, the cell associated with the horizontal edge has orthogonal diagonals $\left(\mathbf{n}_{j} \cdot \mathbf{n}_{j}^{\prime}=0\right)$; it thus checks the geometrical Hypotheses 5.5 and 5.6. As regards the diamond-cell associated with the oblique edge, its splitting into 

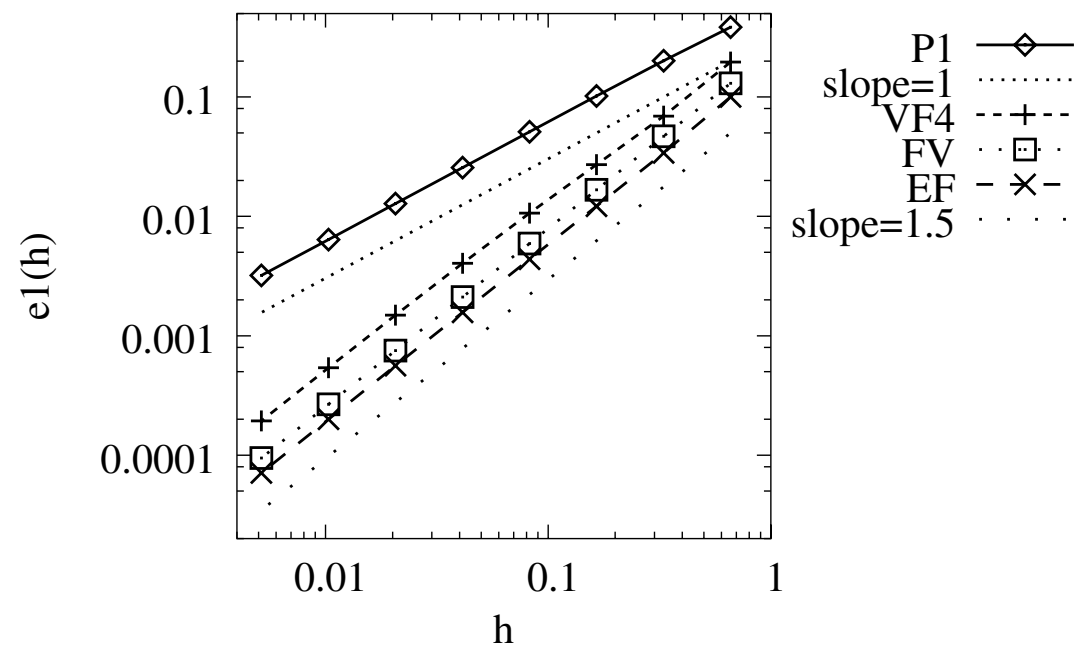

FiguRE 17. Discrete $\mathrm{H}_{0}^{1}$ convergence on the family of triangular grids refined by subdivision.

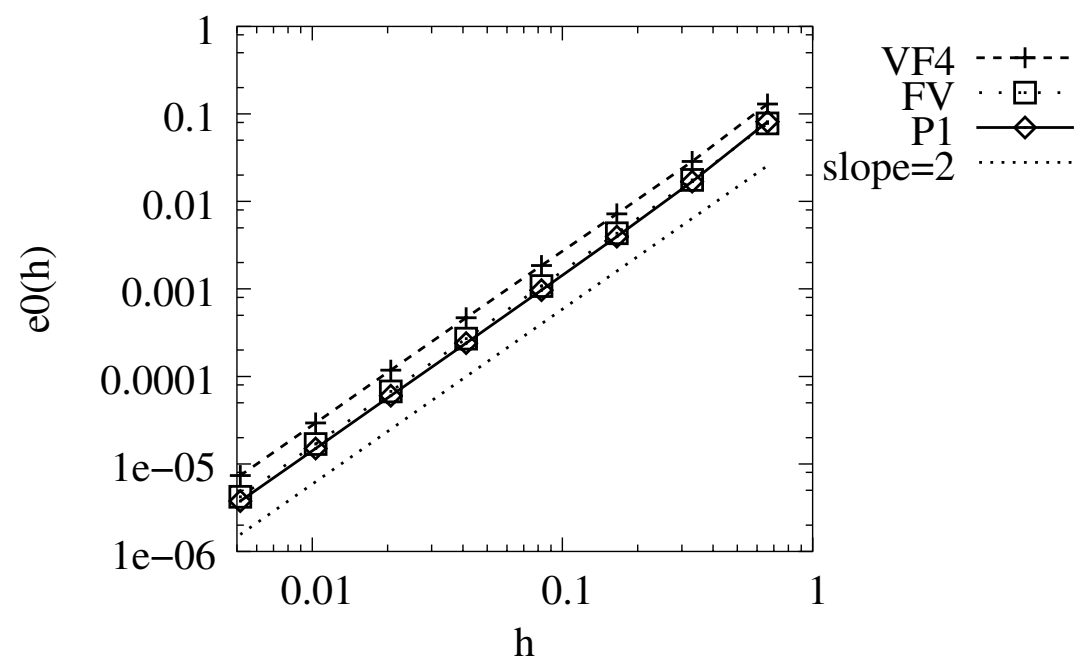

FIGURE 18. Discrete $\mathrm{L}^{2}$ convergence on the family of triangular grids refined by subdivision.

$\left(D_{j, 1}, D_{j, 2}\right)$ gives a maximum angle $\tau_{1}=\tau_{2}$ which tends to $\frac{\pi}{2}$, which implies that this diamond-cell checks the geometrical Hypothesis 5.6 although the angle between its diagonals tends to 0. In Figure 21, the cells associated with the horizontal and vertical edges also check Hypothesis 5.6. On the other hand, the cell associated with the oblique edge is such that $\sin \tau_{1} \sim 3 / 2^{n}$ and $\sin \tau_{2} \sim 5 / 2^{n}$. These two angles thus tend toward $\pi$ when $n$ grows, and this diamond-cell thus does not check Hypothesis 5.6. In addition, $h / \sin \tau_{2} \leq C$, where $C$ does not depend on the grid. If one reconsiders the error estimates obtained in (81) and (92), and if one denotes by $D_{h}$ the union of the diamond-cells on which this problem is encountered, we obtain

$$
\left|\hat{\phi}-\phi_{h}\right|_{1, h} \leq C\left(\left.h|| f\right|_{0, \Omega}+|\hat{\phi}|_{2, D_{h}}\right) .
$$




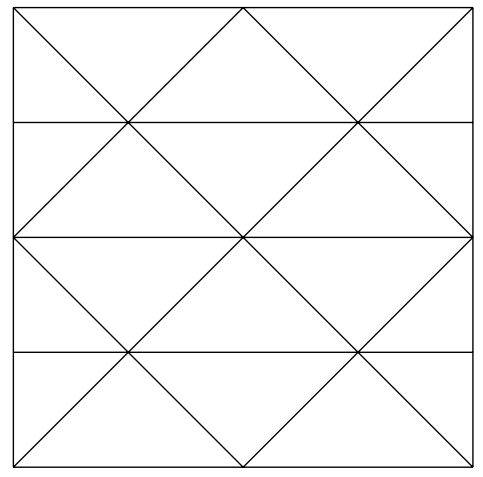

(a) mesh with flat triangles, $n=1$

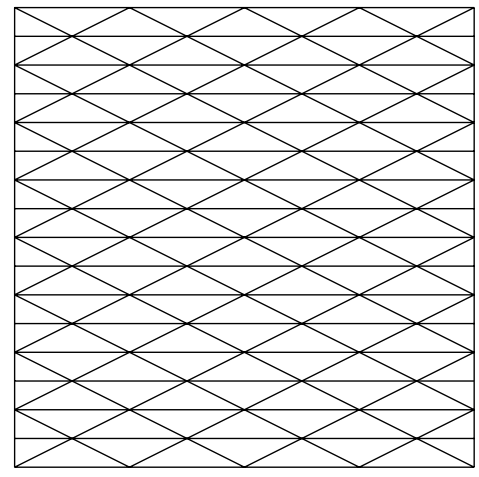

(b) mesh with flat triangles, $n=2$

Figure 19. Meshes with degenerating flat triangles.



FIGURE 20. Interior diamond-cells associated with the degenerating grids.

But since $\hat{\phi}$ is in this example of class $\mathcal{C}^{2}(\bar{\Omega})$, and since the area of $D_{h}$ is of order $C h$, with a constant independent of $h$, we can bound $|\hat{\phi}|_{2, D_{h}}$ by $C \sqrt{h}$, which explains the order 0.5 observed in Figure 22 . On the other hand, the order 1.5 remains unexplained.

As far as the VF4 scheme is concerned, we remark the non-convergence of the scheme. This may be related to the fact that, when switching from a refinement level $n$ to a level $n+1$, the distance $\left|A_{j}^{\prime}\right|$ between the IOBs of two adjacent triangles $T_{i_{1}(j)}$ and $T_{i_{2}(j)}$ remains approximatively constant, due to the degeneracy of the grids. Thus the difference quotient $\frac{\phi_{i_{1}(j)}-\phi_{i_{2}(j)}}{\left|A_{j}^{\prime}\right|}$ never converges toward $\nabla \hat{\phi} \cdot \mathbf{n}$.

We observe in Figure 23 a second-order convergence of the proposed finite volume method in the $\mathrm{L}^{2}$ norm.

Finally, we consider two applications on non-conforming meshes. First, we consider the non-conforming family of meshes constructed in the following way. Let $n$ be a non-zero integer. We split $\Omega$ into $\left(2^{n}+1\right) \times\left(2^{n}+1\right)$ identical squares. Then, we refine this mesh locally "in a chess-board way"; this means that every other square is itself divided into $2^{n} \times 2^{n}$ identical sub-squares. We choose here $n \in[1 ; 5]_{\mathbb{N}}$. Figure 24 displays the first two of these meshes. Of course, this family of meshes is not of practical use, but constitutes in our opinion a good choice to test the applicability of the presented method on arbitrarily locally refined non-conforming meshes. The quantity $e 1(h)$ is displayed in logarithmic scale in Figure 25, as well as a reference straight line with a slope equal to one. The quantity $e 0(h)$ is displayed in logarithmic scale in Figure 26, as well as a reference straight line with a slope equal to two. We observe, on this family of non-conforming, locally refined meshes, a first-order convergence in the discrete $\mathrm{H}_{0}^{1}$ norm and a second-order convergence in the discrete $\mathrm{L}^{2}$ norm. 


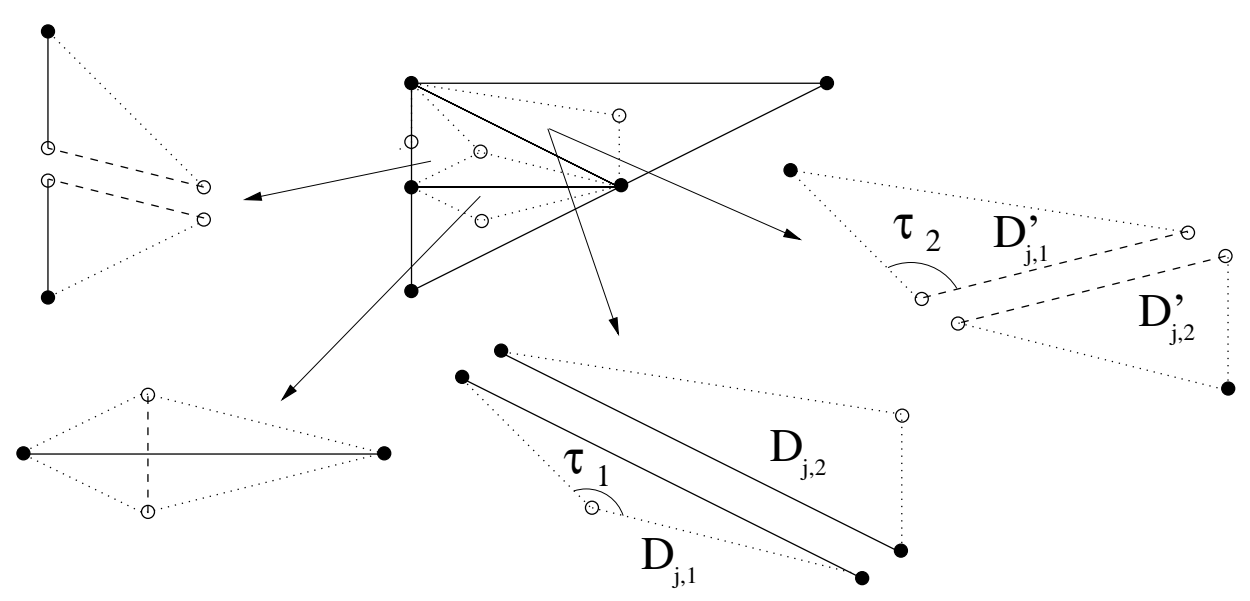

FiguRE 21. Diamond-cells close to the edge associated with the degenerating grids.

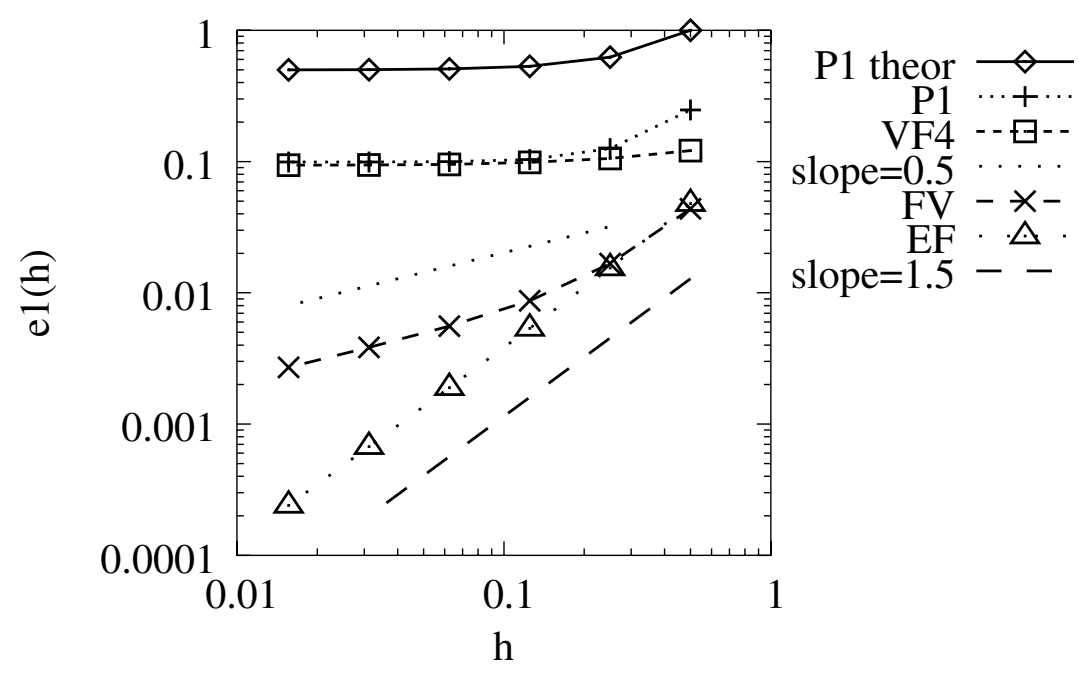

Figure 22. Convergence in the discrete $\mathrm{H}_{0}^{1}$ norm on the family of degenerating triangular grids.

As a second and more realistic application, we consider an example inspired from [13], where the authors consider the following "multi-scale" problem. Let $\Omega=[-1,1]^{2}$ and let us choose the following data (together with homogeneous boundary conditions):

$$
f=2 k^{2} \pi^{2} \cos (k \pi x) \cos (k \pi y)-4 \eta \chi(r) \exp \left(1 / \varepsilon^{2}\right) \exp \left[-1 /\left(\varepsilon^{2}-r^{2}\right)\right] \frac{r^{2}+r^{4}-\varepsilon^{4}}{\left(\varepsilon^{2}-r^{2}\right)^{4}}
$$

with $r=\sqrt{x^{2}+y^{2}}$ and $\chi(r)=1$ if $r \leq \varepsilon$ while $\chi(r)=0$ if $r>\varepsilon$. We fix $k=1 / 2, \eta=10$ and $\varepsilon=1 / 4$. The exact solution $\hat{\phi}$ of this problem is given by

$$
\hat{\phi}=\cos (k \pi x) \cos (k \pi y)+\eta \chi(r) \exp \left(1 / \varepsilon^{2}\right) \exp \left[-1 /\left(\varepsilon^{2}-r^{2}\right)\right],
$$

thus it is smooth away from the origin and displays a strong peak in the neighbourhood of $(0,0)$. Like in [13], we consider the subdomain $\omega=[-1 / 4,1 / 4]^{2}$ and $\Omega \backslash \omega$ is uniformly meshed with squares of size $S$, while 


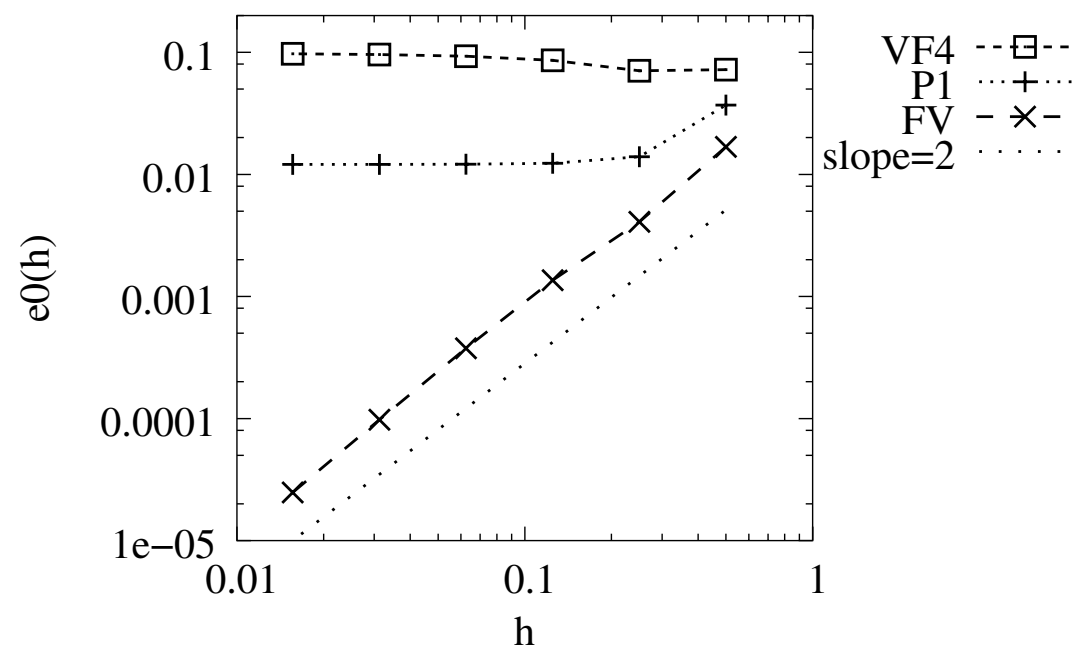

Figure 23. Convergence in the discrete $\mathrm{L}^{2}$ norm on the family of degenerating triangular grids.

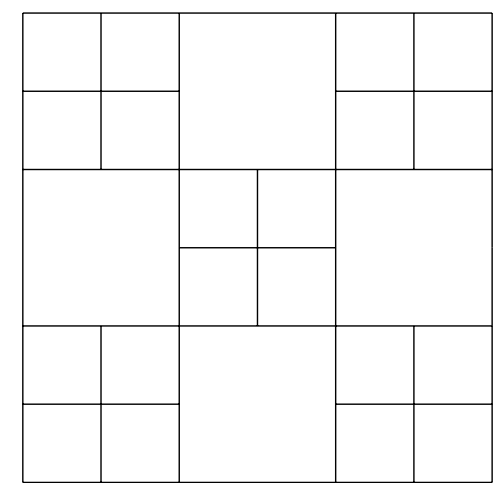

(a) non-conforming mesh, $n=1$

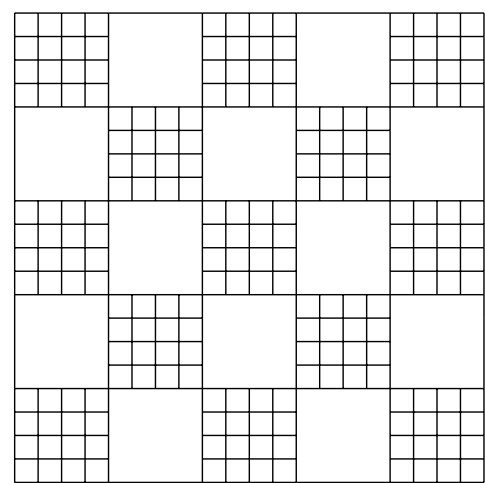

(b) non-conforming mesh, $n=2$

FiguRE 24. Non conforming locally refined meshes.

$\omega$ is uniformly meshed with squares of size $s=S / 2^{p}$. Thus, for $p \geq 1$ the mesh is non-conforming. The mesh corresponding to $S=1 / 8$ and $S / s=4$ is displayed in Figure 27. First, we try to find what could be the optimal ratio $S / s$ by two tests. We first fix $S=1 / 16$ and let $s$ tend to 0 as long as $e 1_{F E}$ significantly decreases. Figure 28 shows that it is worth refining the mesh covering $\omega$ until the ratio $S / s=16$, and that further refinements are less efficient. Second, we fix $s=1 / 256$ and coarsen the mesh covering $\Omega \backslash \omega$ until the error $e 1_{F E}$ starts to increase significantly. There again, as seen in Figure 28, it seems that coarsening up to $S / s=16$ does not increase the error much, and that further coarsening starts to deteriorate the solution. So we choose to fix $S / s=16$ and then let $S$ and $s$ tend to 0 . The only diamond-cells which are not parallelograms are located at the boundaries of $\Omega$ and $\omega$ and are thus included in a region whose area is bounded by $C h$, where the constant $C$ does not depend on $h$. Thus, the expected rate of convergence in the $\mathrm{H}_{0}^{1}$ norm is 1.5 , which seems to be (at least asymptotically) the case in Figure 29. As far as the $\mathrm{L}^{2}$ norm is concerned, Figure 30 shows a second order convergence. We think this example shows that the method proposed here allows to deal in a particularly simple way with non-conforming locally refined meshes, while maintaining the optimal order of convergence. Indeed, for this type of meshes, it is known (see e.g. [23] and the references therein in the context of domain 


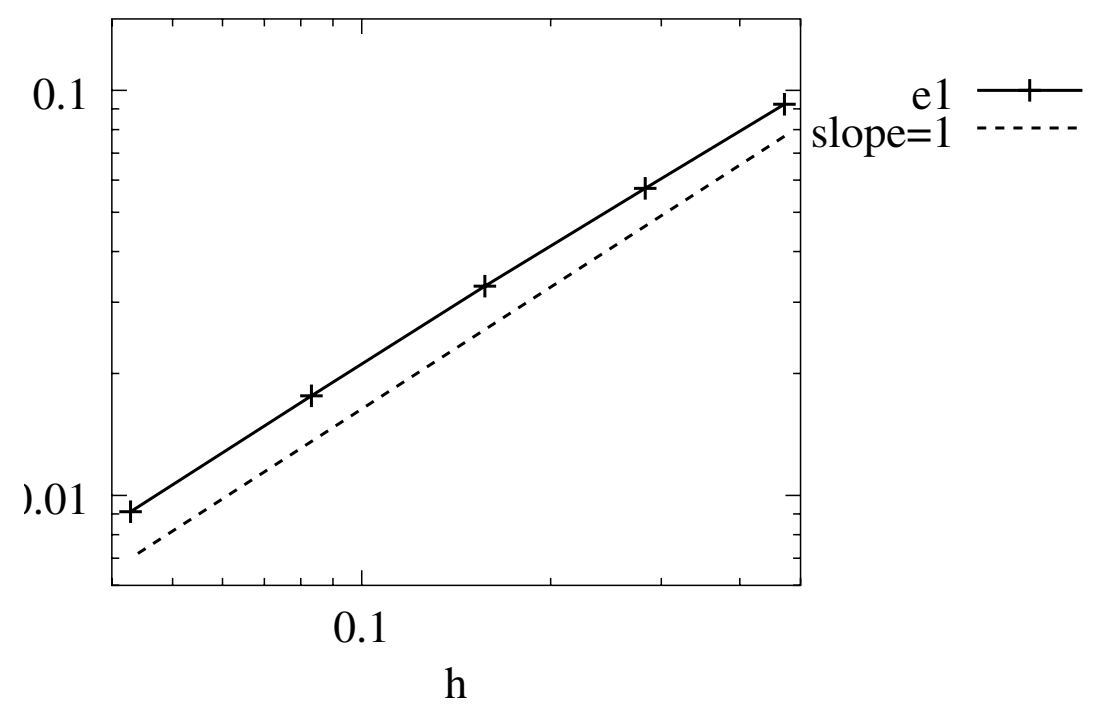

Figure 25. Convergence in the discrete $\mathrm{H}_{0}^{1}$ norm on the family of locally refined nonconforming meshes.

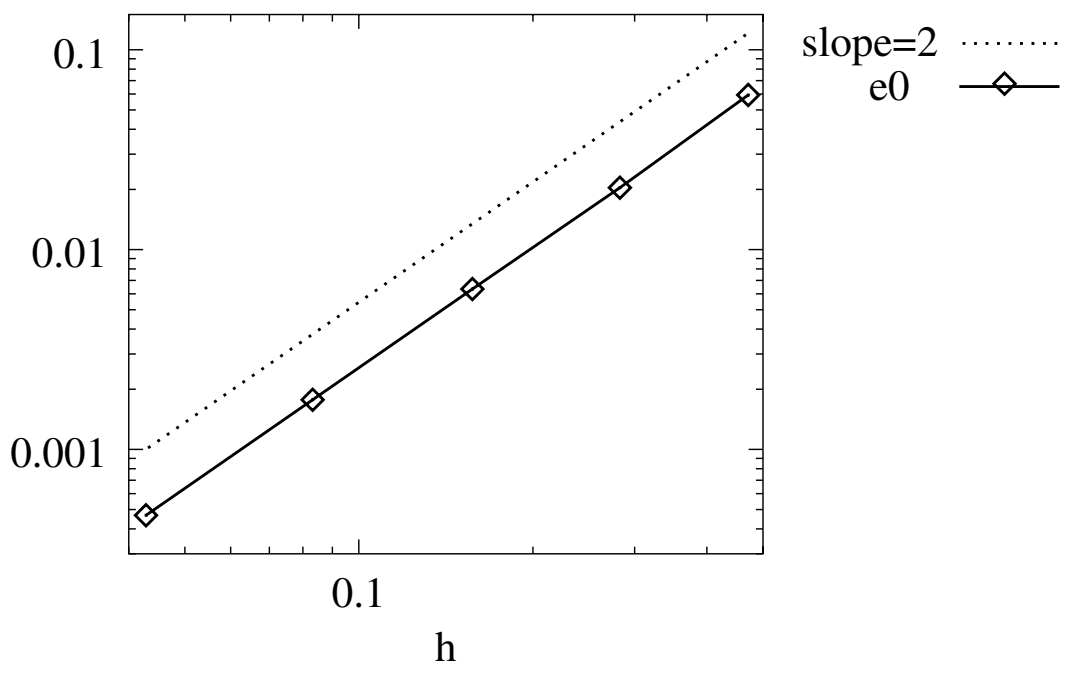

Figure 26. Convergence in the discrete $\mathrm{L}^{2}$ norm on the family of locally refined nonconforming meshes.

decomposition on non-matching grids) that the standard two point flux approximation at the interface between the coarse and fine meshes leads to a deterioration of the order of convergence $\left(0.5\right.$ in the $\mathrm{H}^{1}$ norm $)$.

\section{Conclusion}

In this article, we have presented a finite volume method based on the integration of the Laplace equation on both a primal, almost arbitrary, two-dimensional mesh and on a dual mesh. For this, we have defined a discrete gradient operator on the diamond-cells of the mesh and a discrete divergence operator on the primal and dual cells, such that these two discrete operators are linked by a discrete Green formula. Thanks to this property, 


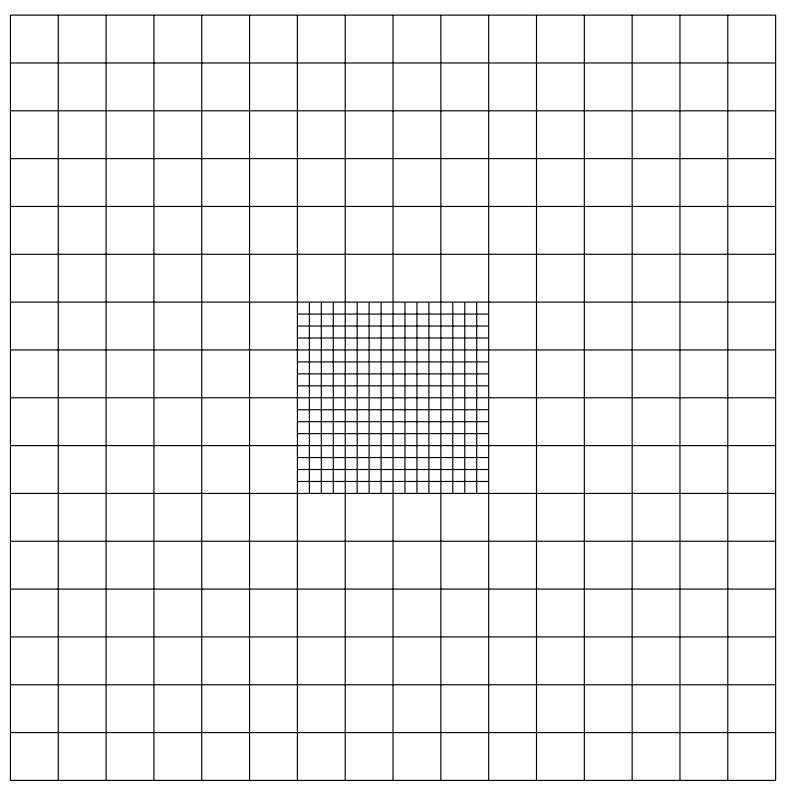

Figure 27. Non conforming mesh for the multi-scale problem with $S=1 / 8$ and $S / s=4$.



FiguRE 28. $\mathrm{H}_{0}^{1}$ norm of the error for the multi-scale problem with respect to the local refinement ratio.

the finite volume method may be interpreted under a variational form. We proved its equivalence with a finite element method whose basis functions are $P^{1}$ over each diamond cell, and continuous at the midpoints of the interfaces between the diamond cells. Then, we have proved error estimates in two discrete $\mathrm{H}_{0}^{1}$ norms, using either a finite volume approach or a finite element approach. We obtain a convergence of order one under the hypothesis that the maximum angle of one of the two couples of triangles obtained by partition of a diamond-cell according to one or the other of its diagonals is bounded uniformly by an angle strictly lower than $\pi$. A first order convergence in the $\mathrm{L}^{2}$ norm is proved under this same hypothesis in the general case. Better estimates can be obtained on families of homothetically refined meshes, under the hypothesis of an additional regularity 


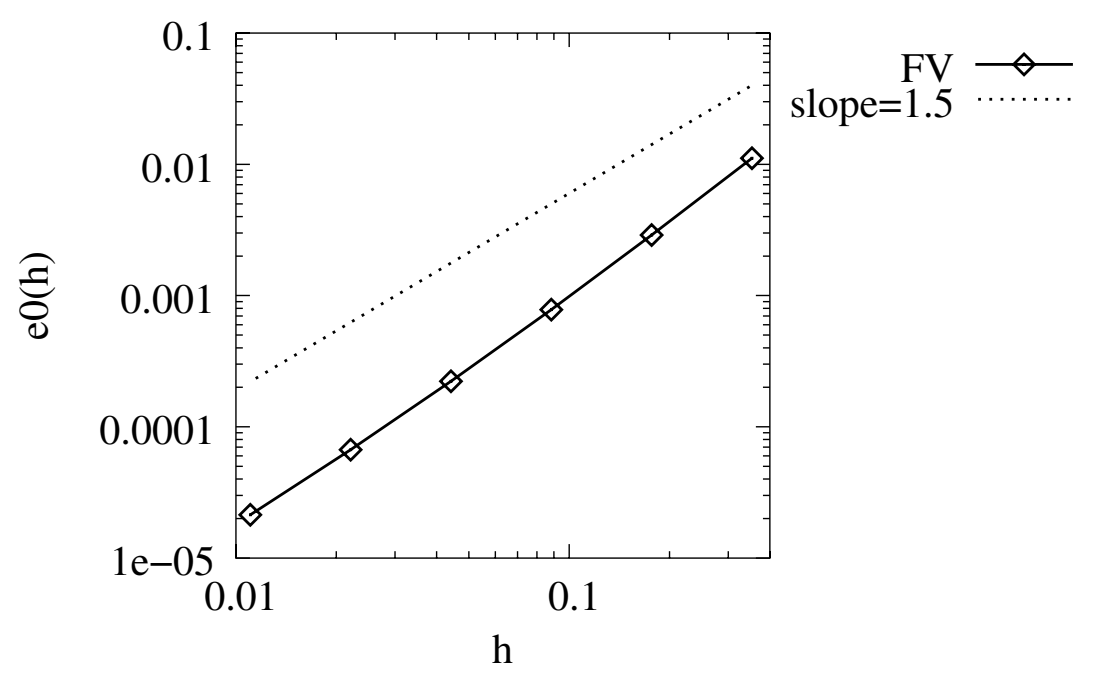

Figure 29. Convergence in the discrete $\mathrm{H}_{0}^{1}$ norm for the multi-scale problem with fixed $S / s$.

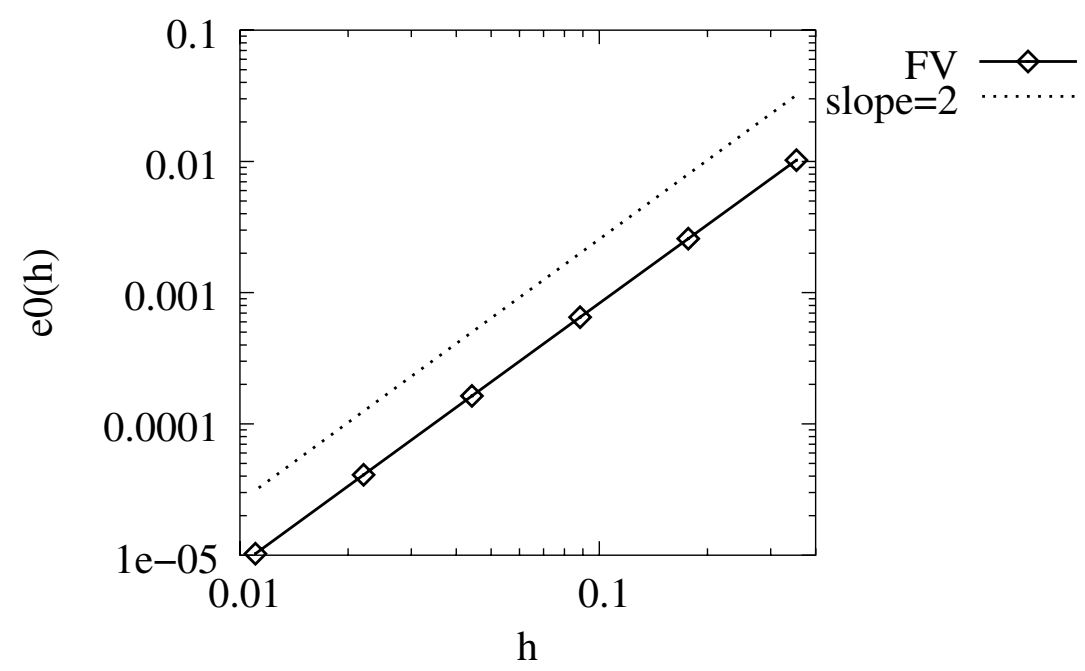

Figure 30. Convergence in discrete $\mathrm{L}^{2}$ norm for the multi-scale problem with fixed $S / s$.

of the data. Indeed, we obtain a superconvergence at the order $1.5-\alpha$ in the $\mathrm{H}_{0}^{1}$ norm, while a $2-\alpha$ order is proved in the $\mathrm{L}^{2}$ norm, for any $\alpha>0$. The numerical tests carried out show that in general one cannot hope for better than first-order convergence in the discrete $\mathrm{H}_{0}^{1}$ norm, but confirm the superconvergence on homothetically refined grids. Convergence with the order two in the discrete $\mathrm{L}^{2}$ norm is also observed for very general grids; this phenomenon remains to be studied. Another point worth mentioning is that the gradients of the solution are particularly well approximated by the method, even on degenerating grids. Finally, a numerical example has shown that this method behaves particularly well on non-conforming locally refined meshes. The next step in this direction is the construction of an a posteriori error estimator well adapted to the method, in order to efficiently refine meshes for a given problem. More general anisotropic and/or non-linear diffusion operators were studied numerically in [15]; the setting of these problems into the formalism of discrete operators on general grids, as well as the coupling with convection terms is the subject of current investigations. 
Acknowledgements. The authors are pleased to thank Marie-Hélène Vignal, Philippe Villedieu and Jean-Paul Vila for interesting and fruitful discussions on the subject

\section{REFERENCES}

[1] G. Acosta and R.G. Durán, The maximum angle condition for mixed and nonconforming elements: application to the Stokes equations. SIAM J. Numer. Anal. 37 (1999) 18-36.

[2] I. Babuška and A.K. Aziz, On the angle condition in the finite element method. SIAM J. Numer. Anal. 13 (1976) $214-226$.

[3] J. Baranger, J.-F. Maitre and F. Oudin, Connection between finite volume and mixed finite element methods. RAIRO Modél. Math. Anal Numér. 30 (1996) 445-465.

[4] S. Boivin, F. Cayré and J.-M. Hérard, A finite volume method to solve the Navier-Stokes equations for incompressible flows on unstructured meshes. Int. J. Therm. Sci. 39 (2000) 806-825.

[5] P.G. Ciarlet, Basic error estimates for elliptic problems, in Handbook of Numerical Analysis Vol. 2, P.G. Ciarlet and J.-L. Lions, Eds., Amsterdam North-Holland/Elsevier (1991) 17-351.

[6] Y. Coudière, J.-P. Vila and P. Villedieu, Convergence rate of a finite volume scheme for a two dimensional convection-diffusion problem. ESAIM: M2AN 33 (1999) 493-516.

[7] Y. Coudière and P. Villedieu, Convergence rate of a finite volume scheme for the linear convection-diffusion equation on locally refined meshes. ESAIM: M2AN 34 (2000) 1123-1149.

[8] K. Domelevo and P. Omnes, Construction et analyse numérique d'une méthode de volumes finis pour l'équation de Laplace sur des maillages bidimensionnels presque quelconques (in French), Rapport CEA (2004).

[9] R. Eymard, T. Gallouët and R. Herbin, Handbook of Numerical Analysis Vol. 7, P.G. Ciarlet and J.-L. Lions, Eds., NorthHolland/Elsevier, Amsterdam (2000) 713-1020.

[10] R. Eymard, T. Gallouët and R. Herbin, Finite volume approximation of elliptic problems and convergence of an approximate gradient. Appl. Numer. Math. 37 (2001) 31-53.

[11] I. Faille, A control volume method to solve an elliptic equation on a two-dimensional irregular meshing. Comput. Methods Appl. Mech. Engrg. 100 (1991) 275-290.

[12] T. Gallouët, R. Herbin and M.-H. Vignal, Error estimates for the approximate finite volume solution of convection diffusion equations with general boundary conditions. SIAM J. Numer. Anal. 37 (2000) 1935-1972.

[13] R. Glowinski, J. He, J. Rappaz and J. Wagner, A multi-domain method for solving numerically multi-scale elliptic problems. C. R. Acad. Sci. Paris Ser. I Math 338 (2004) 741-746.

[14] R. Herbin, An error estimate for a finite volume scheme for a diffusion-convection problem on a triangular mesh. Numer. Methods Partial Differential Equations 11 (1995) 165-173.

[15] F. Hermeline, A finite volume method for the approximation of diffusion operators on distorted meshes. J. Comput. Phys. 160 (2000) 481-499.

[16] J.M. Hyman and M. Shashkov, Adjoint operators for the natural discretizations of the divergence, gradient, and curl on logically rectangular grids. Appl. Numer. Math. 25 (1997) 413-442.

[17] J.M. Hyman and M. Shashkov, Natural discretizations for the divergence, gradient, and curl on logically rectangular grids. Comput. Math. Appl. 33 (1997) 81-104.

[18] P. Jamet, Estimations d'erreur pour des éléments finis droits presque dégénérés. RAIRO Anal. numér. 10 (1976) 43-61.

[19] L. Klinger, J.B. Vos and K. Appert, A simplified gradient evaluation on non-orthogonal meshes; application to a plasma torch simulation method. Comput. Fluids 33 (2004) 643-654.

[20] I.D. Mishev, Finite volume methods on Voronoi meshes. Numer. Methods Partial Differential Equations 14 (1998) $193-212$.

[21] L.E. Payne and H.F. Weinberger, An optimal Poincaré inequality for convex domains. Arch. Rational Mech. Anal. 5 (1960) $286-292$.

[22] P.-A. Raviart and J.-M. Thomas, A mixed finite element method for second order elliptic problems, in Mathematical aspects of the finite element method, I. Galligani and E. Magenes, Eds., Springer-Verlag, New-York. Lecture Notes in Math. 606 (1977) 292-315.

[23] L. Saas, I. Faille, F. Nataf and F. Willien, Domain decomposition for a finite volume method on non-matching grids. $C$. $R$. Acad. Sci. Paris Ser. I Math. 338 (2004) 407-412.

[24] G. Strang, Variational crimes in the finite element method, in The mathematical foundations of the finite element method with applications to partial differential equations, A.K. Aziz Ed., Academic Press, New York (1972) 689-710.

[25] R. Vanselow and H.P. Scheffler, Convergence analysis of a finite volume method via a new nonconforming finite element method. Numer. Methods Partial Differential Equations 14 (1998) 213-231.

[26] Special issue on the simulation of transport around a nuclear waste disposal site: the Couplex test cases. Computat. Geosci. 8 (2004). 